\title{
Coupling of flavonoid initiation sites with monolignols studied by density functional theory
}

Laura Berstis, ${ }^{\mathrm{a}, \mathrm{b}}$ Thomas Elder, ${ }^{\mathrm{c}}$ Richard Dixon, ${ }^{\mathrm{d}}$ Michael Crowley, ${ }^{\mathrm{e} *}$ and Gregg T. Beckham ${ }^{\mathrm{e} *}$

a. Biosciences Center, National Renewable Energy Laboratory, 15013 West Denver Parkway, Golden CO 80401

b. Current address: University of Fribourg, Department of Biology, Chemin du museé 10, 1700 Fribourg, Switzerland

c. USDA-Forest Service, Southern Research Station, 521 Devall Drive, Auburn AL 36849

d. BioDiscovery Institute and Department of Biological Sciences, University of North Texas, Union Circle \#305220, Denton TX 76203

e. Renewable Resources and Enabling Sciences Center, National Renewable Energy Laboratory, 15013 West Denver Parkway Golden CO 80401

Corresponding authors: Email: Gregg.Beckham@nrel.gov

Michael.Crowley@nrel.gov

\section{Supplementary Information Table of Contents}

\begin{tabular}{|c|l|}
\multicolumn{1}{|c|}{ Page: } & S.I. Material \\
\hline S2 & $\begin{array}{l}\text { Table S1. Bond dissociation enthalpies of flavonoid-monolignol dimers } \\
\text { with threo and erythro stereochemistry, for (A) relaxed geometries of } \\
\text { radical bond dissociation products, and (B) bond coordinate energies for } \\
\text { radical products frozen in the dimer conformation. }\end{array}$ \\
\hline S3 & $\begin{array}{l}\text { Table S2. Tabulation of dimer BDE (kcal/mol) as a function of the } \\
\text { monolignol (columns) and flavonoid (rows), presented as the average of } \\
\text { threo and erythro isomers. }\end{array}$ \\
\hline S4 & $\begin{array}{l}\text { Fig. S1. Correlation of stereoisomer enthalpy, } \Delta \mathrm{H}(\text { threo-erythro), with } \\
\text { stereoisomer bond dissociation enthalpy } \Delta \mathrm{BDE}(\text { threo-erythro) in } \\
\text { kcal/mol. }\end{array}$ \\
\hline S5 & $\begin{array}{l}\text { Table S3. Final optimized coordinates of flavonoid and monolignol } \\
\text { monomers and dimers with uM06-2X/Def2-TZVPP. }\end{array}$ \\
\hline
\end{tabular}

Total pages: 59

Figures: 1

Tables: 3 
Table S1. Bond dissociation enthalpies of flavonoid-monolignol dimers with threo and erythro stereochemistry, for (A) relaxed geometries of radical bond dissociation products, and (B) bond coordinate energies for radical products frozen in the dimer conformation.

A. Bond Dissociation Enthalpies, $\mathrm{kcal} / \mathrm{mol}$ (Relaxed geometries of radical bond dissociation products)

\begin{tabular}{|c|c|c|c|c|c|c|c|c|c|c|c|c|c|}
\hline & \multicolumn{2}{|c|}{ coumaryl } & \multicolumn{2}{|c|}{ caffeyl } & \multicolumn{2}{|c|}{$50 \mathrm{OH}$} & \multicolumn{4}{|c|}{$\begin{array}{l}\text { feruoyl, } \\
\text { saturated }\end{array}$} & \multirow{2}{*}{$\begin{array}{c}\text { ferulate } \\
\text { condensed }\end{array}$} & \multicolumn{2}{|c|}{ sinapyl } \\
\hline & threo & erythro & threo & erythro & threo & erythro & threo & erythro & threo & erythro & & threo & erythro \\
\hline tricin & 69.62 & 66.17 & 70.71 & 66.68 & 72.06 & 65.60 & 70.73 & 69.96 & 56.22 & 58.14 & -71.40 & 68.52 & 68.11 \\
\hline apigenin & 72.33 & 72.59 & 70.67 & 73.24 & 71.02 & 74.50 & 70.85 & 73.91 & 63.46 & 65.17 & -81.94 & 70.95 & 73.28 \\
\hline luteolin & 65.80 & 66.34 & 66.35 & 66.11 & 66.93 & 66.30 & 66.49 & 66.31 & 60.16 & 58.88 & -73.82 & 66.46 & 67.29 \\
\hline chrysoeriol & 69.65 & 68.40 & 69.52 & 70.78 & 70.19 & 68.08 & 66.34 & 72.50 & 59.37 & 61.11 & -77.66 & 70.72 & 73.67 \\
\hline catechin $(R, S)$ & 76.42 & 72.24 & 76.79 & 76.95 & 74.07 & 75.46 & 73.97 & 74.00 & 63.53 & 62.65 & -80.16 & 78.31 & 75.42 \\
\hline catechin $(S, R)$ & 75.46 & 68.44 & 78.26 & 75.10 & 76.75 & 75.30 & 73.93 & 71.59 & 61.40 & 62.48 & -80.15 & 75.66 & 75.66 \\
\hline epicatechin $(R, R)$ & 76.74 & 74.55 & 78.55 & 76.89 & 77.76 & 75.29 & 77.45 & 76.31 & 62.92 & 67.02 & -81.51 & 79.71 & 78.20 \\
\hline epicatechin $(S, S)$ & 65.22 & 76.74 & 76.08 & 73.35 & 75.93 & 75.18 & 76.77 & 75.80 & 61.36 & 64.81 & -76.44 & 78.00 & 75.77 \\
\hline epigallocatechin & 63.93 & 65.28 & 72.83 & 66.98 & 74.23 & 67.67 & 74.10 & 67.00 & 55.50 & 52.31 & -66.89 & 74.21 & 67.57 \\
\hline gallate & 67.93 & 67.92 & 75.27 & 69.72 & 75.20 & 70.93 & 77.62 & 65.83 & 56.93 & 59.45 & -77.60 & 77.80 & 71.94 \\
\hline \multicolumn{14}{|c|}{ Bond Coordinate Energies, $\mathrm{kcal} / \mathrm{mol}$ (Frozen geometries of radical bond dissociation products) } \\
\hline & \multicolumn{2}{|c|}{ coumaryl } & \multicolumn{2}{|c|}{ caffeyl } & \multicolumn{2}{|c|}{$50 \mathrm{OH}$} & \multicolumn{4}{|c|}{$\begin{array}{l}\text { feruoyl, } \\
\text { saturated }\end{array}$} & ferulate & \multicolumn{2}{|c|}{ sinapyl } \\
\hline & threo & erythro & threo & erythro & threo & erythro & threo & erythro & threo & erythro & condensed & threo & erythro \\
\hline tricin & 91.29 & 85.05 & 92.76 & 85.09 & 82.29 & 84.59 & 94.48 & 93.98 & 85.19 & 83.90 & -86.13 & 94.38 & 85.29 \\
\hline apigenin & 88.47 & 89.67 & 86.74 & 87.67 & 86.84 & 87.69 & 86.84 & 90.35 & 84.66 & 86.94 & -92.49 & 86.86 & 91.00 \\
\hline luteolin & 85.89 & 85.96 & 83.88 & 85.84 & 83.97 & 86.54 & 83.94 & 85.98 & 87.19 & 85.72 & -88.82 & 84.35 & 86.10 \\
\hline chrysoeriol & 86.23 & 84.70 & 90.75 & 89.89 & 92.18 & 92.22 & 84.37 & 90.52 & 90.03 & 84.15 & -90.64 & 95.29 & 84.80 \\
\hline catechin $(R, S)$ & 96.67 & 91.47 & 97.98 & 96.53 & 96.70 & 95.74 & 95.82 & 91.49 & 87.43 & 95.38 & -97.23 & 99.34 & 94.91 \\
\hline catechin $(S, R)$ & 95.61 & 91.94 & 99.69 & 94.84 & 98.69 & 95.05 & 94.37 & 92.58 & 90.15 & 93.59 & -100.27 & 97.18 & 97.18 \\
\hline epicatechin $(R, R)$ & 97.14 & 92.47 & 100.74 & 95.31 & 98.87 & 95.63 & 99.53 & 99.53 & 92.05 & 99.14 & -99.65 & 100.95 & 96.75 \\
\hline epicatechin (S,S) & 90.41 & 97.14 & 97.94 & 92.68 & 100.07 & 94.58 & 97.13 & 93.33 & 90.80 & 94.69 & -91.47 & 98.36 & 95.37 \\
\hline $\begin{array}{l}\text { epigallocatechin } \\
\text { epigallocatechin }\end{array}$ & 89.56 & 86.72 & 94.37 & 90.15 & 97.01 & 89.52 & 95.66 & 88.72 & 84.21 & 80.39 & -85.31 & 95.98 & 89.55 \\
\hline gallate & 94.12 & 91.83 & 100.79 & 94.01 & 99.39 & 95.31 & 101.87 & 91.97 & 85.51 & 94.86 & -101.06 & 102.17 & 96.81 \\
\hline
\end{tabular}


Table S2. Tabulation of dimer BDE (kcal/mol) as a function of the monolignol (columns) and flavonoid (rows), presented as the average of threo and erythro isomers.

\begin{tabular}{|c|c|c|c|c|c|c|c|}
\hline \multirow[t]{2}{*}{ Flavonoid type } & \multicolumn{6}{|c|}{ Monolignol type } & \multirow[b]{2}{*}{ sinapyl } \\
\hline & coumaryl & caffeyl & $5 \mathrm{HG}$ & coniferyl & $\begin{array}{l}\text { feruoyl, } \\
\text { saturated }\end{array}$ & ferulate & \\
\hline tricin & 67.9 & 68.7 & 68.3 & 70.3 & 57.2 & 71.4 & 68.8 \\
\hline apigenin & 72.5 & 72 & 72.1 & 72.4 & 64.3 & 81.9 & 72.8 \\
\hline luteolin & 66.1 & 66.2 & 66.9 & 66.4 & 59.5 & 73.8 & 66.6 \\
\hline chrysoeriol & 69 & 70.2 & 72.2 & 69.4 & 60.2 & 77.7 & 69.1 \\
\hline catechin $(\mathrm{R}, \mathrm{S})$ & 74.3 & 76.9 & 74.8 & 74 & 63.1 & 80.2 & 76.9 \\
\hline catechin $(S, R)$ & 71.9 & 76.7 & 76 & 72.8 & 61.9 & 80.2 & 75.7 \\
\hline epicatechin $(R, R)$ & 75.6 & 77.7 & 76.5 & 76.9 & 65 & 81.5 & 79 \\
\hline epicatechin $(S, S)$ & 71 & 74.7 & 75.6 & 76.3 & 63.1 & 76.4 & 76.9 \\
\hline epigallocatechin & 64.6 & 69.9 & 71 & 70.6 & 53.9 & 66.9 & 70.9 \\
\hline $\begin{array}{r}\text { epigallocatechin } \\
\text { gallate }\end{array}$ & 67.9 & 84.6 & 73.1 & 65.8 & 70.9 & 77.6 & 74.9 \\
\hline
\end{tabular}

$\begin{array}{lllllll}\mathrm{BDE}(\mathrm{kcal} / \mathrm{mol}) & <59 & 59-64 & 64-69 & 69-74 & 74-79 & >79\end{array}$ 


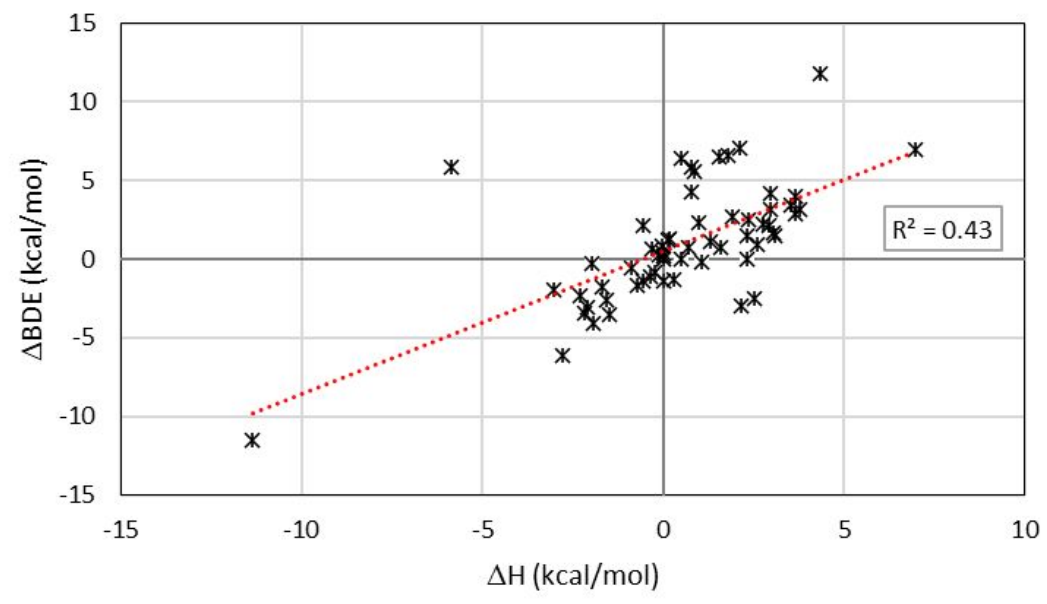

Fig. S1. Correlation of stereoisomer enthalpy, $\Delta \mathrm{H}($ threo-erythro), with stereoisomer bond dissociation enthalpy $\Delta \mathrm{BDE}($ threoerythro) in $\mathrm{kcal} / \mathrm{mol}$. 
Table S3. Final optimized coordinates of flavonoid and monolignol monomers and dimers with uM06-2X/Def2-TZVPP. Field order: $1=$ center \# in molecule, $2=$ atom proton \#, $3=0$ position-holder, $4=X$-coordinate, $5=Y$-coordinate, $6=Z$-coordinate.

\begin{tabular}{|c|c|c|c|c|c|c|c|c|c|c|c|}
\hline & & & & & & 10no & & & & & \\
\hline apig & $\operatorname{nin}$ & & & & & lute & & & & & \\
\hline 1 & 6 & 0 & 4.161914 & -0.550218 & 0.024660 & 1 & 6 & 0 & 4.359834 & 0.709077 & -0.023662 \\
\hline 2 & 6 & 0 & 3.457639 & 0.641169 & -0.030755 & 2 & 6 & 0 & 3.741201 & -0.527451 & 0.061272 \\
\hline 3 & 6 & 0 & 2.044423 & 0.627016 & -0.022998 & 3 & 6 & 0 & 2.330707 & -0.614646 & 0.037503 \\
\hline 4 & 6 & 0 & 1.395955 & -0.606650 & 0.039322 & 4 & 6 & 0 & 1.597102 & 0.566741 & -0.070794 \\
\hline 5 & 6 & 0 & 2.079301 & -1.805827 & 0.095594 & 5 & 6 & 0 & 2.194358 & 1.809365 & -0.157123 \\
\hline 6 & 6 & 0 & 3.467576 & -1.756158 & 0.087240 & 6 & 6 & 0 & 3.582519 & 1.859626 & -0.131820 \\
\hline 7 & 6 & 0 & 1.273657 & 1.855318 & -0.084789 & 7 & 6 & 0 & 1.648778 & -1.892770 & 0.129236 \\
\hline 8 & 6 & 0 & -0.711790 & 0.447669 & -0.005613 & 8 & 6 & 0 & -0.430957 & -0.635142 & -0.016199 \\
\hline 9 & 6 & 0 & -0.161229 & 1.676324 & -0.082193 & 9 & 6 & 0 & 0.205039 & -1.817754 & 0.106479 \\
\hline 10 & 1 & 0 & 5.243556 & -0.523353 & 0.018580 & 10 & 1 & 0 & 5.440474 & 0.759473 & -0.004838 \\
\hline 11 & 1 & 0 & 1.557840 & -2.748586 & 0.148273 & 11 & 1 & 0 & 1.608661 & 2.710740 & -0.246384 \\
\hline 12 & 6 & 0 & -2.151433 & 0.154933 & 0.008753 & 12 & 6 & 0 & -1.887513 & -0.448033 & -0.057428 \\
\hline 13 & 6 & 0 & -3.082385 & 1.141989 & 0.342052 & 13 & 6 & 0 & -2.436092 & 0.808239 & 0.216916 \\
\hline 14 & 6 & 0 & -2.617192 & -1.117232 & -0.317174 & 14 & 6 & 0 & -2.739414 & -1.506086 & -0.361749 \\
\hline 15 & 6 & 0 & -4.435201 & 0.874348 & 0.338183 & 15 & 6 & 0 & -3.801961 & 0.985463 & 0.199129 \\
\hline 16 & 1 & 0 & -2.744751 & 2.127736 & 0.630229 & 16 & 1 & 0 & -1.787922 & 1.642826 & 0.450038 \\
\hline 17 & 6 & 0 & -3.972852 & -1.394105 & -0.327080 & 17 & 6 & 0 & -4.112065 & -1.324477 & -0.381146 \\
\hline 18 & 1 & 0 & -1.912058 & -1.894652 & -0.572448 & 18 & 1 & 0 & -2.333619 & -2.476175 & -0.609055 \\
\hline 19 & 6 & 0 & -4.886373 & -0.398393 & -0.001224 & 19 & 6 & 0 & -4.654140 & -0.082233 & -0.098376 \\
\hline 20 & 8 & 0 & 0.036632 & -0.675168 & 0.053552 & 20 & 8 & 0 & 0.236449 & 0.537144 & -0.101929 \\
\hline 21 & 8 & 0 & 1.808602 & 2.967518 & -0.144498 & 21 & 8 & 0 & 2.261465 & -2.961034 & 0.229122 \\
\hline 22 & 8 & 0 & 4.117536 & -2.941047 & 0.143452 & 22 & 8 & 0 & 4.146669 & 3.085952 & -0.218216 \\
\hline 23 & 1 & 0 & 5.066905 & -2.794322 & 0.134185 & 23 & 1 & 0 & 5.103967 & 3.008227 & -0.195678 \\
\hline 24 & 8 & 0 & 4.118616 & 1.794275 & -0.091565 & 24 & 8 & 0 & 4.481449 & -1.627737 & 0.165676 \\
\hline 25 & 1 & 0 & 3.441417 & 2.514204 & -0.125773 & 25 & 1 & 0 & 3.856628 & -2.393036 & 0.213976 \\
\hline 26 & 8 & 0 & -6.224228 & -0.608696 & 0.009643 & 26 & 8 & 0 & -5.991411 & 0.095723 & -0.117120 \\
\hline 27 & 1 & 0 & -6.418282 & -1.518174 & -0.231087 & 27 & 1 & 0 & -6.184434 & 1.015881 & 0.095960 \\
\hline 28 & 1 & 0 & -0.781240 & 2.554324 & -0.165415 & 28 & 1 & 0 & -0.352341 & -2.734537 & 0.210864 \\
\hline 29 & 1 & 0 & -5.158878 & 1.631667 & 0.603814 & 29 & 1 & 0 & -4.782215 & -2.136903 & -0.623865 \\
\hline 30 & 1 & 0 & -4.323828 & -2.385022 & -0.588900 & 30 & 8 & 0 & -4.430267 & 2.170627 & 0.460574 \\
\hline & & & & & & 31 & 1 & 0 & -3.788424 & 2.858593 & 0.650706 \\
\hline chry & oer & & & & & trici & & & & & \\
\hline 1 & 6 & 0 & 4.485543 & 0.865236 & 0.000029 & 1 & 6 & 0 & 5.017667 & 0.424288 & 0.123940 \\
\hline 2 & 6 & 0 & 3.967455 & -0.419619 & -0.000173 & 2 & 6 & 0 & 4.301113 & -0.739754 & -0.100175 \\
\hline 3 & 6 & 0 & 2.568087 & -0.616160 & -0.000070 & 3 & 6 & 0 & 2.888181 & -0.710728 & -0.098010 \\
\hline 4 & 6 & 0 & 1.741883 & 0.506836 & 0.000178 & 4 & 6 & 0 & 2.251964 & 0.509106 & 0.131688 \\
\hline 5 & 6 & 0 & 2.238071 & 1.796418 & 0.000361 & 5 & 6 & 0 & 2.947923 & 1.681000 & 0.357909 \\
\hline 6 & 6 & 0 & 3.618181 & 1.955001 & 0.000293 & 6 & 6 & 0 & 4.335525 & 1.617457 & 0.349943 \\
\hline 7 & 6 & 0 & 1.987959 & -1.946340 & -0.000176 & 7 & 6 & 0 & 2.104634 & -1.909291 & -0.335507 \\
\hline 8 & 6 & 0 & -0.189313 & -0.851792 & 0.000047 & 8 & 6 & 0 & 0.132605 & -0.503747 & -0.073443 \\
\hline 9 & 6 & 0 & 0.543534 & -1.984862 & -0.000182 & 9 & 6 & 0 & 0.672769 & -1.715424 & -0.319498 \\
\hline 10 & 1 & 0 & 5.559097 & 1.000186 & -0.000045 & 10 & 1 & 0 & 6.098983 & 0.386206 & 0.119705 \\
\hline 11 & 1 & 0 & 1.581995 & 2.652606 & 0.000557 & 11 & 1 & 0 & 2.436320 & 2.613159 & 0.539088 \\
\hline 12 & 6 & 0 & -1.657895 & -0.769667 & 0.000111 & 12 & 6 & 0 & -1.304353 & -0.200163 & -0.028272 \\
\hline 13 & 6 & 0 & -2.444291 & -1.916769 & 0.000442 & 13 & 6 & 0 & -2.230904 & -1.224584 & 0.163103 \\
\hline 14 & 6 & 0 & -2.282643 & 0.485041 & -0.000167 & 14 & 6 & 0 & -1.735589 & 1.114548 & -0.188902 \\
\hline 15 & 6 & 0 & -3.826934 & -1.827868 & 0.000487 & 15 & 6 & 0 & -3.590479 & -0.946743 & 0.183033 \\
\hline 16 & 1 & 0 & -1.988546 & -2.895434 & 0.000691 & 16 & 1 & 0 & -1.887312 & -2.233739 & 0.324633 \\
\hline 17 & 6 & 0 & -3.658854 & 0.572608 & -0.000126 & 17 & 6 & 0 & -3.093507 & 1.386411 & -0.170514 \\
\hline 18 & 1 & 0 & -1.677748 & 1.377424 & -0.000421 & 18 & 1 & 0 & -1.013039 & 1.901225 & -0.333185 \\
\hline 19 & 6 & 0 & -4.443007 & -0.590373 & 0.000202 & 19 & 6 & 0 & -4.028639 & 0.368962 & 0.011075 \\
\hline 20 & 8 & 0 & 0.388205 & 0.370749 & 0.000240 & 20 & 8 & 0 & 0.893831 & 0.590508 & 0.148158 \\
\hline 21 & 8 & 0 & 2.683760 & -2.967911 & -0.000283 & 21 & 8 & 0 & 2.629220 & -3.008610 & -0.545739 \\
\hline 22 & 8 & 0 & 4.082894 & 3.225643 & 0.000480 & 22 & 8 & 0 & 4.997464 & 2.775897 & 0.573858 \\
\hline 23 & 1 & 0 & 5.043595 & 3.222364 & 0.000409 & 23 & 1 & 0 & 5.945245 & 2.620891 & 0.549428 \\
\hline 24 & 8 & 0 & 4.792964 & -1.462777 & -0.000497 & 24 & 8 & 0 & 4.949838 & -1.880807 & -0.317715 \\
\hline 25 & 1 & 0 & 4.229746 & -2.276171 & -0.000774 & 25 & 1 & 0 & 4.264410 & -2.580860 & -0.454635 \\
\hline 26 & 8 & 0 & -5.787974 & -0.500497 & 0.000246 & 26 & 8 & 0 & -5.348869 & 0.636097 & 0.032695 \\
\hline 27 & 1 & 0 & -6.029215 & 0.433300 & 0.000021 & 27 & 1 & 0 & -5.458871 & 1.585761 & -0.093918 \\
\hline 28 & 8 & 0 & -4.379941 & 1.727524 & -0.000382 & 28 & 8 & 0 & -3.651270 & 2.620153 & -0.321802 \\
\hline 29 & 6 & 0 & -3.664096 & 2.945143 & -0.000726 & 29 & 8 & 0 & -4.564701 & -1.862197 & 0.370005 \\
\hline 30 & 1 & 0 & -3.039431 & 3.027919 & -0.892377 & 30 & 6 & 0 & -2.778015 & 3.714780 & -0.505575 \\
\hline 31 & 1 & 0 & -4.406323 & 3.737236 & -0.000892 & 31 & 1 & 0 & -2.183985 & 3.590490 & -1.413072 \\
\hline 32 & 1 & 0 & -3.039347 & 3.028372 & 0.890825 & 32 & 1 & 0 & -3.406403 & 4.594971 & -0.599891 \\
\hline 33 & 1 & 0 & 0.070805 & -2.952891 & -0.000358 & 33 & 1 & 0 & -2.112952 & 3.829933 & 0.352521 \\
\hline 34 & 1 & 0 & -4.445972 & -2.713747 & 0.000751 & 34 & 6 & 0 & -4.173811 & -3.205824 & 0.542749 \\
\hline & & & & & & 35 & 1 & 0 & -5.090978 & -3.773443 & 0.665502 \\
\hline & & & & & & 36 & 1 & 0 & -3.631823 & -3.574691 & -0.331386 \\
\hline & & & & & & 37 & 1 & 0 & -3.550926 & -3.325180 & 1.432424 \\
\hline & & & & & & 38 & 1 & 0 & 0.044729 & -2.564796 & -0.534150 \\
\hline & & & & & & cin & & 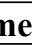 & & & \\
\hline trici & $5-($ & glu & coside & & & trici & 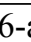 & IUI & ose-8-g & lucosid & \\
\hline 1 & 6 & 0 & -1.973472 & 3.009445 & -0.984548 & 1 & 6 & 0 & 2.611246 & -0.969414 & -0.194166 \\
\hline 2 & 6 & 0 & -1.844483 & 1.636910 & -0.941403 & 2 & 6 & 0 & 1.917660 & -2.121465 & -0.517810 \\
\hline 3 & 6 & 0 & -0.594652 & 1.028386 & -0.680993 & 3 & 6 & 0 & 0.513153 & -2.112525 & -0.479638 \\
\hline 4 & 6 & 0 & 0.485966 & 1.897416 & -0.475442 & 4 & 6 & 0 & -0.148537 & -0.942637 & -0.110690 \\
\hline 5 & 6 & 0 & 0.373670 & 3.278164 & -0.509711 & 5 & 6 & 0 & 0.537818 & 0.226303 & 0.200267 \\
\hline 6 & 6 & 0 & -0.865573 & 3.828677 & -0.770183 & 6 & 6 & 0 & 1.930116 & 0.196230 & 0.176240 \\
\hline 7 & 6 & 0 & -0.341235 & -0.425660 & -0.666833 & 7 & 6 & 0 & -0.250198 & -3.309679 & -0.796076 \\
\hline 8 & 6 & 0 & 2.012658 & 0.117038 & -0.243394 & 8 & 6 & 0 & -2.239266 & -2.000963 & -0.293905 \\
\hline 9 & 6 & 0 & 1.045403 & -0.794972 & -0.443263 & 9 & 6 & 0 & -1.679386 & -3.172056 & -0.668596 \\
\hline 10 & 1 & 0 & -2.954823 & 3.424346 & -1.172417 & 10 & 6 & 0 & -3.672866 & -1.769284 & -0.089217 \\
\hline 11 & 1 & 0 & 1.239189 & 3.902258 & -0.347979 & 11 & 6 & 0 & -4.084768 & -0.764524 & 0.790927 \\
\hline 12 & 6 & 0 & 3.432016 & -0.168334 & 0.010318 & 12 & 6 & 0 & -4.614828 & -2.559907 & -0.736671 \\
\hline 13 & 6 & 0 & 3.958604 & -1.429891 & -0.290853 & 13 & 6 & 0 & -5.433696 & -0.582432 & 1.019989 \\
\hline
\end{tabular}




\begin{tabular}{|c|c|}
\hline 14 & 6 \\
\hline 15 & 6 \\
\hline 16 & 1 \\
\hline 17 & 6 \\
\hline 18 & 1 \\
\hline 19 & 6 \\
\hline 20 & 8 \\
\hline 21 & 8 \\
\hline 22 & 8 \\
\hline 23 & 1 \\
\hline 24 & 8 \\
\hline 25 & 8 \\
\hline 26 & 1 \\
\hline 27 & 8 \\
\hline 28 & 8 \\
\hline 29 & 6 \\
\hline 30 & 1 \\
\hline 31 & 1 \\
\hline 32 & 1 \\
\hline 33 & 6 \\
\hline 34 & 1 \\
\hline 35 & 1 \\
\hline 36 & 1 \\
\hline 37 & 1 \\
\hline 38 & 6 \\
\hline 39 & 6 \\
\hline 40 & 1 \\
\hline 41 & 6 \\
\hline 42 & 6 \\
\hline 43 & 6 \\
\hline 44 & 8 \\
\hline 45 & 8 \\
\hline 46 & 1 \\
\hline 47 & 1 \\
\hline 48 & 8 \\
\hline 49 & 1 \\
\hline 50 & 8 \\
\hline 51 & 1 \\
\hline 52 & 1 \\
\hline 53 & 1 \\
\hline 54 & 6 \\
\hline 55 & 1 \\
\hline 56 & 1 \\
\hline 57 & 1 \\
\hline 58 & 8 \\
\hline 59 & 1 \\
\hline & \\
\hline & \\
\hline & \\
\hline & \\
\hline & \\
\hline & \\
\hline & \\
\hline & \\
\hline & \\
\hline & \\
\hline & \\
\hline & \\
\hline & \\
\hline & \\
\hline & \\
\hline & \\
\hline & \\
\hline & \\
\hline & \\
\hline trici & 8 \\
\hline 1 & 6 \\
\hline 2 & 6 \\
\hline 3 & 6 \\
\hline 4 & 6 \\
\hline 5 & 6 \\
\hline 6 & 6 \\
\hline 7 & 6 \\
\hline 8 & 6 \\
\hline 9 & 6 \\
\hline 10 & 6 \\
\hline 11 & 6 \\
\hline 12 & 6 \\
\hline 13 & 6 \\
\hline 14 & 1 \\
\hline 15 & 6 \\
\hline 16 & 1 \\
\hline 17 & 6 \\
\hline 18 & 8 \\
\hline 19 & 8 \\
\hline 20 & 8 \\
\hline 21 & 1 \\
\hline 22 & 8 \\
\hline 23 & 1 \\
\hline 24 & 8 \\
\hline 25 & 1 \\
\hline 26 & 8 \\
\hline 27 & 8 \\
\hline 28 & 6 \\
\hline 29 & 1 \\
\hline 30 & 1 \\
\hline 31 & 1 \\
\hline
\end{tabular}




\begin{tabular}{|c|c|c|c|}
\hline & & & \\
\hline 32 & 6 & 0 & 4.764027 \\
\hline 33 & 1 & 0 & 5.280743 \\
\hline 34 & 1 & 0 & 4.038370 \\
\hline 35 & 1 & 0 & 4.249996 \\
\hline 36 & 1 & 0 & 2.622988 \\
\hline 37 & 8 & 0 & -3.652281 \\
\hline 38 & 8 & 0 & 0.144953 \\
\hline 39 & 6 & 0 & -4.650087 \\
\hline 40 & 6 & 0 & -4.546576 \\
\hline 41 & 1 & 0 & -5.592670 \\
\hline 42 & 6 & 0 & -5.499251 \\
\hline 43 & 6 & 0 & -5.616604 \\
\hline 44 & 6 & 0 & -5.459881 \\
\hline 45 & 6 & 0 & 0.157624 \\
\hline 46 & 1 & 0 & 0.428582 \\
\hline 47 & 6 & 0 & 0.724203 \\
\hline 48 & 6 & 0 & -1.570653 \\
\hline 49 & 6 & 0 & -0.596514 \\
\hline 50 & 1 & 0 & 0.532105 \\
\hline 51 & 8 & 0 & -4.552237 \\
\hline 52 & 1 & 0 & -1.753643 \\
\hline 53 & 1 & 0 & -4.492077 \\
\hline 54 & 1 & 0 & -6.600358 \\
\hline 55 & 1 & 0 & -3.562347 \\
\hline 56 & 1 & 0 & -6.509763 \\
\hline 57 & 8 & 0 & -4.777966 \\
\hline 58 & 1 & 0 & -3.993166 \\
\hline 59 & 8 & 0 & -5.536956 \\
\hline 60 & 1 & 0 & -5.511016 \\
\hline 61 & 8 & 0 & -6.514796 \\
\hline 62 & 1 & 0 & -6.578692 \\
\hline 63 & 6 & 0 & -5.138568 \\
\hline 64 & 1 & 0 & -5.864033 \\
\hline 65 & 1 & 0 & -5.209972 \\
\hline 66 & 8 & 0 & -3.869727 \\
\hline 67 & 1 & 0 & -3.180654 \\
\hline 68 & 1 & 0 & -2.520873 \\
\hline 69 & 1 & 0 & -0.982748 \\
\hline 70 & 6 & 0 & 1.214283 \\
\hline 71 & 1 & 0 & 1.409333 \\
\hline 72 & 8 & 0 & -1.095720 \\
\hline 73 & 8 & 0 & 2.430372 \\
\hline 74 & 1 & 0 & 2.214958 \\
\hline 75 & 8 & 0 & 1.663485 \\
\hline 76 & 1 & 0 & 2.523626 \\
\hline 77 & 8 & 0 & -0.361948 \\
\hline 78 & 1 & 0 & -1.197990 \\
\hline & & & \\
\hline cour & ary & le & hol \\
\hline 1 & 6 & 0 & 2.825024 \\
\hline 2 & 6 & 0 & 2.197541 \\
\hline 3 & 6 & 0 & 0.812505 \\
\hline 4 & 6 & 0 & 0.024697 \\
\hline 5 & 6 & 0 & 0.681445 \\
\hline 6 & 6 & 0 & 2.059128 \\
\hline 7 & 1 & 0 & 2.789431 \\
\hline 8 & 1 & 0 & 0.330857 \\
\hline 9 & 1 & 0 & 0.105205 \\
\hline 10 & 1 & 0 & 2.564298 \\
\hline 11 & 6 & 0 & -1.438016 \\
\hline 12 & 1 & 0 & -1.813998 \\
\hline 13 & 6 & 0 & -2.323713 \\
\hline 14 & 1 & 0 & -1.996899 \\
\hline 15 & 6 & 0 & -3.810300 \\
\hline 16 & 1 & 0 & -4.270892 \\
\hline 17 & 1 & 0 & -4.208006 \\
\hline 18 & 8 & 0 & -4.223061 \\
\hline 19 & 1 & 0 & -3.903227 \\
\hline 20 & 8 & 0 & 4.177959 \\
\hline 21 & 1 & 0 & 4.594176 \\
\hline & & & \\
\hline $5 \mathrm{HC}$ & alc & & \\
\hline 1 & 6 & 0 & 2.247421 \\
\hline 2 & 6 & 0 & 1.431134 \\
\hline 3 & 6 & 0 & 0.060911 \\
\hline 4 & 6 & 0 & -0.509962 \\
\hline 5 & 6 & 0 & 0.310361 \\
\hline 6 & 6 & 0 & 1.686291 \\
\hline 7 & 1 & 0 & -0.564880 \\
\hline 8 & 1 & 0 & -0.106665 \\
\hline 9 & 6 & 0 & -1.960011 \\
\hline 10 & 1 & 0 & -2.265030 \\
\hline 11 & 6 & 0 & -2.912554 \\
\hline 12 & 1 & 0 & -2.670619 \\
\hline 13 & 6 & 0 & -4.370359 \\
\hline 14 & 1 & 0 & -4.803202 \\
\hline 15 & 1 & 0 & -4.511522 \\
\hline 16 & 8 & 0 & -5.106608 \\
\hline 17 & 1 & 0 & -4.710435 \\
\hline 18 & 8 & 0 & 3.601220 \\
\hline 19 & 1 & 0 & 3.836099 \\
\hline 20 & 8 & 0 & 2.475267 \\
\hline 21 & 1 & 0 & 3.391663 \\
\hline 22 & 8 & 0 & 2.118903 \\
\hline
\end{tabular}




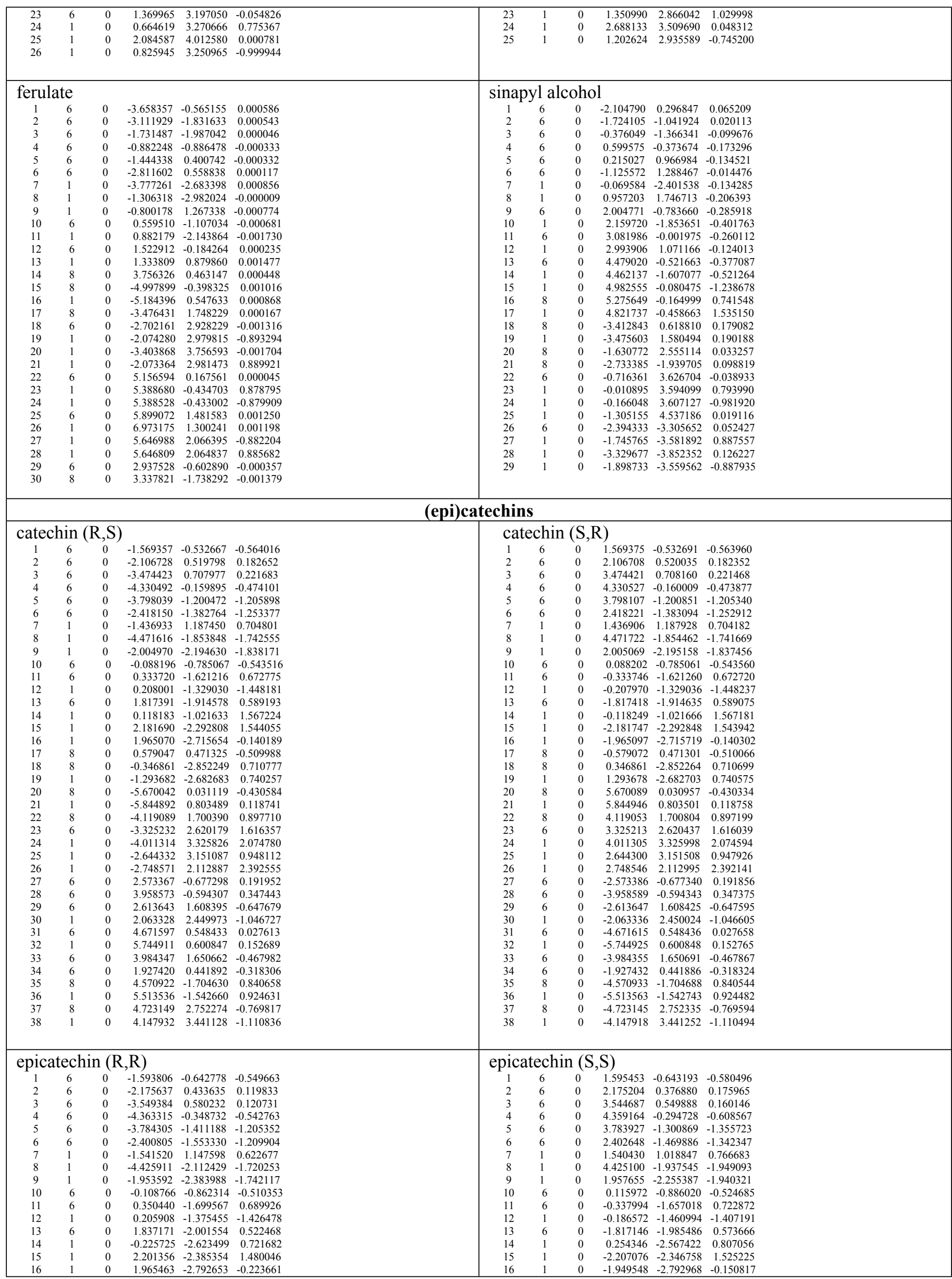




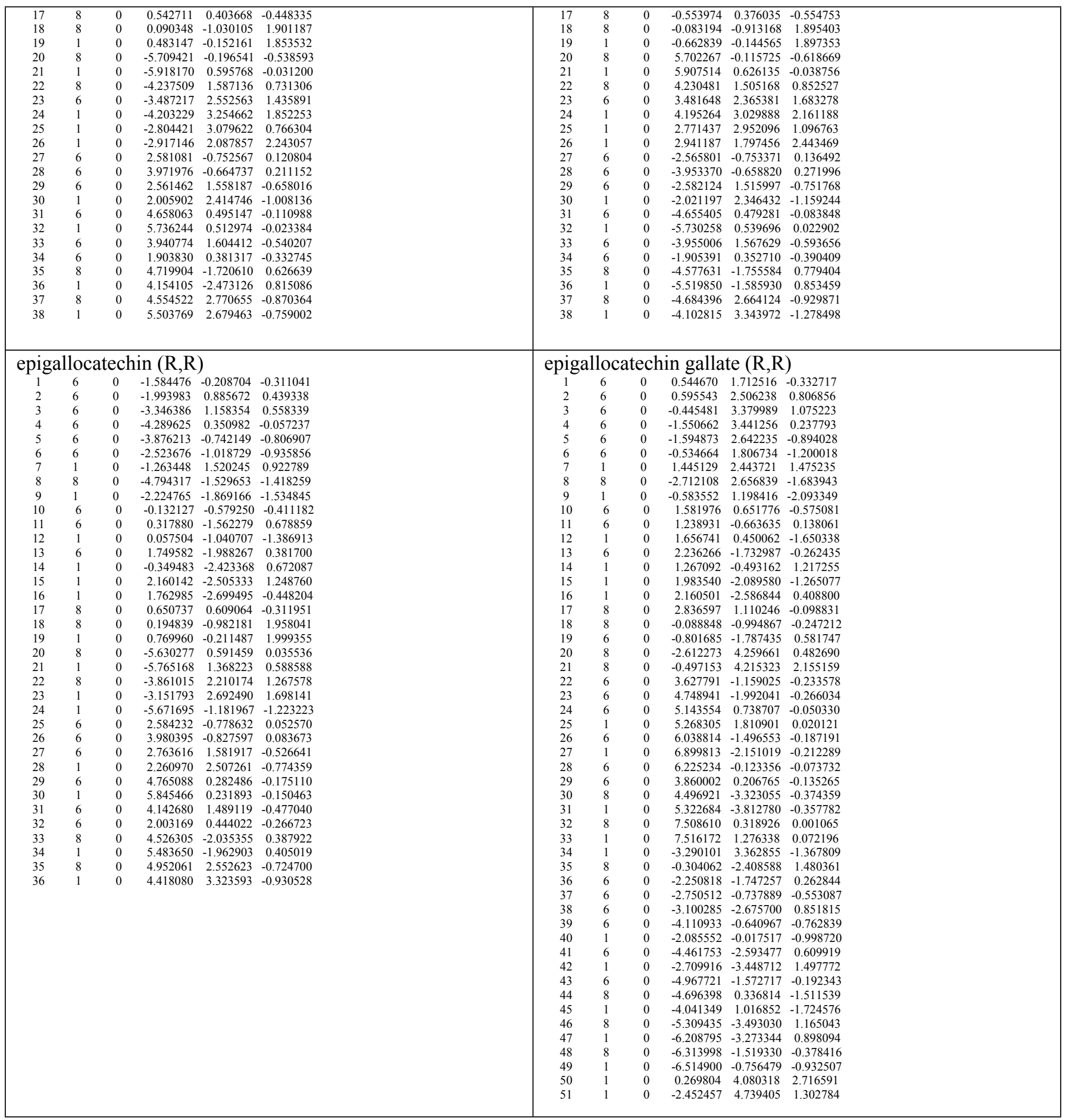

\begin{tabular}{|c|c|c|c|c|c|c|c|c|c|c|c|}
\hline \multicolumn{12}{|c|}{ Flavone-Lignin Dimers: } \\
\hline \multicolumn{6}{|c|}{ H-(S,R)-luteolin } & \multicolumn{6}{|c|}{ H-(S,S)-luteolin } \\
\hline 1 & 6 & 0 & -7.401837 & 0.246522 & 1.131463 & 1 & 6 & 0 & 7.444383 & 0.100806 & 0.356306 \\
\hline 2 & 6 & 0 & -6.767527 & 1.045024 & 0.194825 & 2 & 6 & 0 & 6.637916 & 1.084529 & -0.190713 \\
\hline 3 & 6 & 0 & -5.421680 & 0.790581 & -0.154545 & 3 & 6 & 0 & 5.234175 & 0.919097 & -0.206225 \\
\hline 4 & 6 & 0 & -4.766888 & -0.273412 & 0.467862 & 4 & 6 & 0 & 4.700241 & -0.250066 & 0.337768 \\
\hline 5 & 6 & 0 & -5.381391 & -1.081728 & 1.404171 & 5 & 6 & 0 & 5.486903 & -1.242886 & 0.888588 \\
\hline 6 & 6 & 0 & -6.704479 & -0.804240 & 1.723797 & 6 & 6 & 0 & 6.862219 & -1.047934 & 0.888152 \\
\hline 7 & 6 & 0 & -4.723939 & 1.609875 & -1.127253 & 7 & 6 & 0 & 4.359085 & 1.926408 & -0.775582 \\
\hline 8 & 6 & 0 & -2.793106 & 0.180530 & -0.733315 & 8 & 6 & 0 & 2.513312 & 0.458289 & -0.172392 \\
\hline 9 & 6 & 0 & -3.347590 & 1.232627 & -1.366935 & 9 & 6 & 0 & 2.948766 & 1.605879 & -0.728690 \\
\hline 10 & 1 & 0 & -8.432371 & 0.453448 & 1.388160 & 10 & 1 & 0 & 8.517134 & 0.241895 & 0.361375 \\
\hline 11 & 1 & 0 & -4.856730 & -1.900904 & 1.870264 & 11 & 1 & 0 & 5.052036 & -2.137024 & 1.306872 \\
\hline 12 & 6 & 0 & -1.405731 & -0.283350 & -0.909132 & 12 & 6 & 0 & 1.103441 & 0.041685 & -0.075185 \\
\hline 13 & 6 & 0 & -0.687625 & 0.055048 & -2.056885 & 13 & 6 & 0 & 0.081034 & 0.989593 & -0.143509 \\
\hline 14 & 6 & 0 & -0.805565 & -1.054771 & 0.079952 & 14 & 6 & 0 & 0.792495 & -1.305453 & 0.077069 \\
\hline 15 & 6 & 0 & 0.616597 & -0.380180 & -2.198128 & 15 & 6 & 0 & -1.236446 & 0.579066 & -0.062882 \\
\hline 16 & 1 & 0 & -1.153598 & 0.620266 & -2.850378 & 16 & 1 & 0 & 0.311976 & 2.041672 & -0.221668 \\
\hline
\end{tabular}




\begin{tabular}{|c|c|c|c|}
\hline 17 & 6 & 0 & 0.510752 \\
\hline 18 & 1 & 0 & -1.343070 \\
\hline 19 & 6 & 0 & 1.216886 \\
\hline 20 & 8 & 0 & -3.472235 \\
\hline 21 & 8 & 0 & -5.263983 \\
\hline 22 & 8 & 0 & -7.288167 \\
\hline 23 & 1 & 0 & -8.196380 \\
\hline 24 & 8 & 0 & -7.431424 \\
\hline 25 & 1 & 0 & -6.808698 \\
\hline 26 & 8 & 0 & 2.488614 \\
\hline 27 & 8 & 0 & 1.047562 \\
\hline 28 & 1 & 0 & -2.768350 \\
\hline 29 & 1 & 0 & 1.181852 \\
\hline 30 & 1 & 0 & 2.017540 \\
\hline 31 & 6 & 0 & 3.565986 \\
\hline 32 & 6 & 0 & 3.706442 \\
\hline 33 & 1 & 0 & 2.777500 \\
\hline 34 & 8 & 0 & 3.802890 \\
\hline 35 & 1 & 0 & 4.410894 \\
\hline 36 & 6 & 0 & 4.850194 \\
\hline 37 & 6 & 0 & 4.737909 \\
\hline 38 & 6 & 0 & 6.034161 \\
\hline 39 & 6 & 0 & 5.780824 \\
\hline 40 & 1 & 0 & 3.811673 \\
\hline 41 & 6 & 0 & 7.089011 \\
\hline 42 & 1 & 0 & 6.134046 \\
\hline 43 & 6 & 0 & $\begin{array}{l}0.136570 \\
6.96794\end{array}$ \\
\hline 44 & 1 & 0 & 8.007241 \\
\hline 45 & 8 & 0 & 7.961821 \\
\hline 46 & 1 & 0 & 8.718773 \\
\hline 47 & 1 & 0 & 5.694804 \\
\hline 48 & 6 & 0 & 4.785903 \\
\hline 49 & 1 & 0 & 5.674507 \\
\hline 50 & 1 & 0 & 4.642131 \\
\hline 51 & 8 & 0 & 5.019636 \\
\hline 52 & 1 & 0 & 4.275061 \\
\hline 53 & 1 & 0 & 3.376852 \\
\hline $\mathrm{H}-(\mathrm{S}$ & R)- & irys & beriol \\
\hline 1 & 6 & 0 & 7.440814 \\
\hline 2 & 6 & 0 & 6.713970 \\
\hline 3 & 6 & 0 & 5.366345 \\
\hline 4 & 6 & 0 & 4.804363 \\
\hline 5 & 6 & 0 & 5.511942 \\
\hline 6 & 6 & 0 & 6.834230 \\
\hline 7 & 6 & 0 & 4.573004 \\
\hline 8 & 6 & 0 & 2.744907 \\
\hline 9 & 6 & 0 & 3.207477 \\
\hline 10 & 1 & 0 & 8.471492 \\
\hline 11 & 1 & 0 & 5.058440 \\
\hline 12 & 6 & 0 & 1.378300 \\
\hline 13 & 6 & 0 & 0.590135 \\
\hline 14 & 6 & 0 & 0.864730 \\
\hline 15 & 6 & 0 & -0.703979 \\
\hline 16 & 1 & 0 & 1.009315 \\
\hline 17 & 6 & 0 & -0.435527 \\
\hline 18 & 1 & 0 & 1.470235 \\
\hline 19 & 6 & 0 & -1.218225 \\
\hline 20 & 8 & 0 & 3.512252 \\
\hline 21 & 8 & 0 & 5.028369 \\
\hline 22 & 8 & 0 & 7.509259 \\
\hline 23 & 1 & 0 & 8.408148 \\
\hline 24 & 8 & 0 & $\begin{array}{l}0.70140 \\
.291412\end{array}$ \\
\hline 25 & 1 & 0 & 6.613222 \\
\hline 26 & 8 & 0 & -2.470902 \\
\hline 27 & 1 & 0 & -0.874794 \\
\hline 28 & 1 & 0 & 2.560176 \\
\hline 29 & 8 & 0 & -1.534055 \\
\hline 30 & 6 & 0 & -3.564471 \\
\hline 31 & 6 & 0 & -3.559057 \\
\hline 32 & 1 & 0 & -2.615912 \\
\hline 33 & 8 & 0 & -3.566260 \\
\hline 34 & 1 & 0 & -4.137391 \\
\hline 35 & 6 & 0 & -4.692229 \\
\hline 36 & 6 & 0 & -4.644505 \\
\hline 37 & 6 & 0 & -5.805095 \\
\hline 38 & 6 & 0 & -5.680447 \\
\hline 39 & 1 & 0 & -3.772970 \\
\hline 40 & 6 & 0 & -6.852534 \\
\hline 41 & 1 & 0 & -5.847714 \\
\hline 42 & 6 & 0 & -6.793703 \\
\hline 43 & 1 & 0 & -7.714573 \\
\hline 44 & 8 & 0 & -7.787702 \\
\hline 45 & 1 & 0 & -8.489862 \\
\hline 46 & 1 & 0 & $\begin{array}{l}-5.4642873 \\
-\end{array}$ \\
\hline 47 & 6 & 0 & -4.797112 \\
\hline 48 & 1 & 0 & -5.697191 \\
\hline 49 & 1 & 0 & -4.768594 \\
\hline 50 & 8 & 0 & -4.876958 \\
\hline 51 & 1 & 0 & -4.089488 \\
\hline 52 & 1 & 0 & -3.488250 \\
\hline 53 & 6 & 0 & -1.036740 \\
\hline 54 & 1 & 0 & -0.169055 \\
\hline 55 & 1 & 0 & -0.764209 \\
\hline 56 & 1 & 0 & -1.841036 \\
\hline
\end{tabular}




\begin{tabular}{|c|c|c|}
\hline 1 & 6 & 0 \\
\hline 2 & 6 & 0 \\
\hline 3 & 6 & 0 \\
\hline 4 & 6 & 0 \\
\hline 5 & 6 & 0 \\
\hline 6 & 6 & 0 \\
\hline 7 & 6 & 0 \\
\hline 8 & 6 & 0 \\
\hline 9 & 6 & 0 \\
\hline 10 & 1 & 0 \\
\hline 11 & 1 & 0 \\
\hline 12 & 6 & 0 \\
\hline 13 & 6 & 0 \\
\hline 14 & 6 & 0 \\
\hline 15 & 6 & 0 \\
\hline 16 & 1 & 0 \\
\hline 17 & 6 & 0 \\
\hline 18 & 1 & 0 \\
\hline 19 & 6 & 0 \\
\hline 20 & 8 & 0 \\
\hline 21 & 8 & 0 \\
\hline 22 & 8 & 0 \\
\hline 23 & 1 & 0 \\
\hline 24 & 8 & 0 \\
\hline 25 & 1 & 0 \\
\hline 26 & 8 & 0 \\
\hline 27 & 1 & 0 \\
\hline 28 & 1 & 0 \\
\hline 29 & 1 & 0 \\
\hline 30 & 6 & 0 \\
\hline 31 & 6 & 0 \\
\hline 32 & 1 & 0 \\
\hline 33 & 8 & 0 \\
\hline 34 & 1 & 0 \\
\hline 35 & 6 & 0 \\
\hline 36 & 6 & 0 \\
\hline 37 & 6 & 0 \\
\hline 38 & 6 & 0 \\
\hline 39 & 1 & 0 \\
\hline 40 & 6 & 0 \\
\hline 41 & 1 & 0 \\
\hline 42 & 6 & 0 \\
\hline 43 & 1 & 0 \\
\hline 44 & 8 & 0 \\
\hline 45 & 1 & 0 \\
\hline 46 & 1 & 0 \\
\hline 47 & 6 & 0 \\
\hline 48 & 1 & 0 \\
\hline 49 & 1 & 0 \\
\hline 50 & 8 & 0 \\
\hline 51 & 1 & 0 \\
\hline 52 & 1 & 0 \\
\hline $\mathrm{H}-(\mathrm{s}$ & R) & IC1 \\
\hline 1 & 6 & 0 \\
\hline 2 & 6 & 0 \\
\hline 3 & 6 & 0 \\
\hline 4 & 6 & 0 \\
\hline 5 & 6 & 0 \\
\hline 6 & 6 & 0 \\
\hline 7 & 6 & 0 \\
\hline 8 & 6 & 0 \\
\hline 9 & 6 & 0 \\
\hline 10 & 1 & 0 \\
\hline 11 & 1 & 0 \\
\hline 12 & 6 & 0 \\
\hline 13 & 6 & 0 \\
\hline 14 & 6 & 0 \\
\hline 15 & 6 & 0 \\
\hline 16 & 1 & 0 \\
\hline 17 & 6 & 0 \\
\hline 18 & 1 & 0 \\
\hline 19 & 6 & 0 \\
\hline 20 & 8 & 0 \\
\hline 21 & 8 & 0 \\
\hline 22 & 8 & 0 \\
\hline 23 & 1 & 0 \\
\hline 24 & 8 & 0 \\
\hline 25 & 1 & 0 \\
\hline 26 & 8 & 0 \\
\hline 27 & 8 & 0 \\
\hline 28 & 1 & 0 \\
\hline 29 & 8 & 0 \\
\hline 30 & 6 & 0 \\
\hline 31 & 6 & 0 \\
\hline 32 & 1 & 0 \\
\hline 33 & 8 & 0 \\
\hline 34 & 1 & 0 \\
\hline 35 & 6 & 0 \\
\hline 36 & 6 & 0 \\
\hline 37 & 6 & 0 \\
\hline 38 & 6 & 0 \\
\hline 39 & 1 & 0 \\
\hline 40 & 6 & 0 \\
\hline 41 & 1 & 0 \\
\hline 42 & 6 & 0 \\
\hline 43 & 1 & 0 \\
\hline
\end{tabular}




\begin{tabular}{|c|c|c|}
\hline 44 & 8 & 0 \\
\hline 45 & 1 & 0 \\
\hline 46 & 1 & 0 \\
\hline 47 & 6 & 0 \\
\hline 48 & 1 & 0 \\
\hline 49 & 1 & 0 \\
\hline 50 & 8 & 0 \\
\hline 51 & 1 & 0 \\
\hline 52 & 1 & 0 \\
\hline 53 & 6 & 0 \\
\hline 54 & 1 & 0 \\
\hline 55 & 1 & 0 \\
\hline 56 & 1 & 0 \\
\hline 57 & 6 & 0 \\
\hline 58 & 1 & 0 \\
\hline 59 & 1 & 0 \\
\hline 60 & 1 & 0 \\
\hline $\mathrm{C}-(\mathrm{S}$ & R) & ig \\
\hline 1 & 6 & 0 \\
\hline 2 & 6 & 0 \\
\hline 3 & 6 & 0 \\
\hline 4 & 6 & 0 \\
\hline 5 & 6 & 0 \\
\hline 6 & 6 & 0 \\
\hline 7 & 6 & 0 \\
\hline 8 & 6 & 0 \\
\hline 9 & 6 & 0 \\
\hline 10 & 1 & 0 \\
\hline 11 & 1 & 0 \\
\hline 12 & 6 & 0 \\
\hline 13 & 6 & 0 \\
\hline 14 & 6 & 0 \\
\hline 15 & 6 & 0 \\
\hline 16 & 1 & 0 \\
\hline 17 & 6 & 0 \\
\hline 18 & 1 & 0 \\
\hline 19 & 6 & 0 \\
\hline 20 & 8 & 0 \\
\hline 21 & 8 & 0 \\
\hline 22 & 8 & 0 \\
\hline 23 & 1 & 0 \\
\hline 24 & 8 & 0 \\
\hline 25 & 1 & 0 \\
\hline 26 & 1 & 0 \\
\hline 27 & 8 & 0 \\
\hline 28 & 1 & 0 \\
\hline 29 & 1 & 0 \\
\hline 30 & 6 & 0 \\
\hline 31 & 6 & 0 \\
\hline 32 & 1 & 0 \\
\hline 33 & 8 & 0 \\
\hline 34 & 1 & 0 \\
\hline 35 & 6 & 0 \\
\hline 36 & 6 & 0 \\
\hline 37 & 6 & 0 \\
\hline 38 & 6 & 0 \\
\hline 39 & 1 & 0 \\
\hline 40 & 6 & 0 \\
\hline 41 & 1 & 0 \\
\hline 42 & 6 & 0 \\
\hline 43 & 1 & 0 \\
\hline 44 & 8 & 0 \\
\hline 45 & 1 & 0 \\
\hline 46 & 8 & 0 \\
\hline 47 & 1 & 0 \\
\hline 48 & 6 & 0 \\
\hline 49 & 1 & 0 \\
\hline 50 & 1 & 0 \\
\hline 51 & 8 & 0 \\
\hline 52 & 1 & 0 \\
\hline 53 & 1 & 0 \\
\hline C-(S & R) & tec \\
\hline 1 & 6 & 0 \\
\hline 2 & 6 & 0 \\
\hline 3 & 6 & 0 \\
\hline 4 & 6 & 0 \\
\hline 5 & 6 & 0 \\
\hline 6 & 6 & 0 \\
\hline 7 & 6 & 0 \\
\hline 8 & 6 & 0 \\
\hline 9 & 6 & 0 \\
\hline 10 & 1 & 0 \\
\hline 11 & 1 & 0 \\
\hline 12 & 6 & 0 \\
\hline 13 & 6 & 0 \\
\hline 14 & 6 & 0 \\
\hline 15 & 6 & 0 \\
\hline 16 & 1 & 0 \\
\hline 17 & 6 & 0 \\
\hline 18 & 1 & 0 \\
\hline 19 & 6 & 0 \\
\hline 20 & 8 & 0 \\
\hline 21 & 8 & 0 \\
\hline 22 & 8 & 0 \\
\hline 23 & 1 & 0 \\
\hline 24 & 8 & 0 \\
\hline
\end{tabular}




\begin{tabular}{|c|}
\hline 25 \\
\hline 26 \\
\hline 27 \\
\hline 28 \\
\hline 29 \\
\hline 30 \\
\hline 31 \\
\hline 32 \\
\hline 33 \\
\hline 34 \\
\hline 35 \\
\hline 36 \\
\hline 37 \\
\hline 38 \\
\hline 39 \\
\hline 40 \\
\hline 41 \\
\hline 42 \\
\hline 43 \\
\hline 44 \\
\hline 45 \\
\hline 46 \\
\hline 47 \\
\hline 48 \\
\hline 49 \\
\hline 50 \\
\hline 51 \\
\hline 52 \\
\hline 53 \\
\hline 54 \\
\hline $\mathrm{C}-(\mathrm{S}$ \\
\hline 1 \\
\hline 2 \\
\hline 3 \\
\hline 4 \\
\hline 5 \\
\hline 6 \\
\hline 7 \\
\hline 8 \\
\hline 9 \\
\hline 10 \\
\hline 11 \\
\hline 12 \\
\hline 13 \\
\hline 14 \\
\hline 15 \\
\hline 16 \\
\hline 17 \\
\hline 18 \\
\hline 19 \\
\hline 20 \\
\hline 21 \\
\hline 22 \\
\hline 23 \\
\hline 24 \\
\hline 25 \\
\hline 26 \\
\hline 27 \\
\hline 28 \\
\hline 29 \\
\hline 30 \\
\hline 31 \\
\hline 32 \\
\hline 33 \\
\hline 34 \\
\hline 35 \\
\hline 36 \\
\hline 37 \\
\hline 38 \\
\hline 39 \\
\hline 40 \\
\hline 41 \\
\hline 42 \\
\hline 43 \\
\hline 44 \\
\hline 45 \\
\hline 46 \\
\hline 47 \\
\hline 48 \\
\hline 49 \\
\hline 50 \\
\hline 51 \\
\hline 52 \\
\hline 53 \\
\hline 54 \\
\hline 55 \\
\hline 56 \\
\hline 57 \\
\hline $\mathrm{C}-(\mathrm{S}$ \\
\hline 1 \\
\hline 2 \\
\hline 3 \\
\hline 4 \\
\hline 5 \\
\hline 6 \\
\hline 7 \\
\hline 8 \\
\hline 9 \\
\hline
\end{tabular}




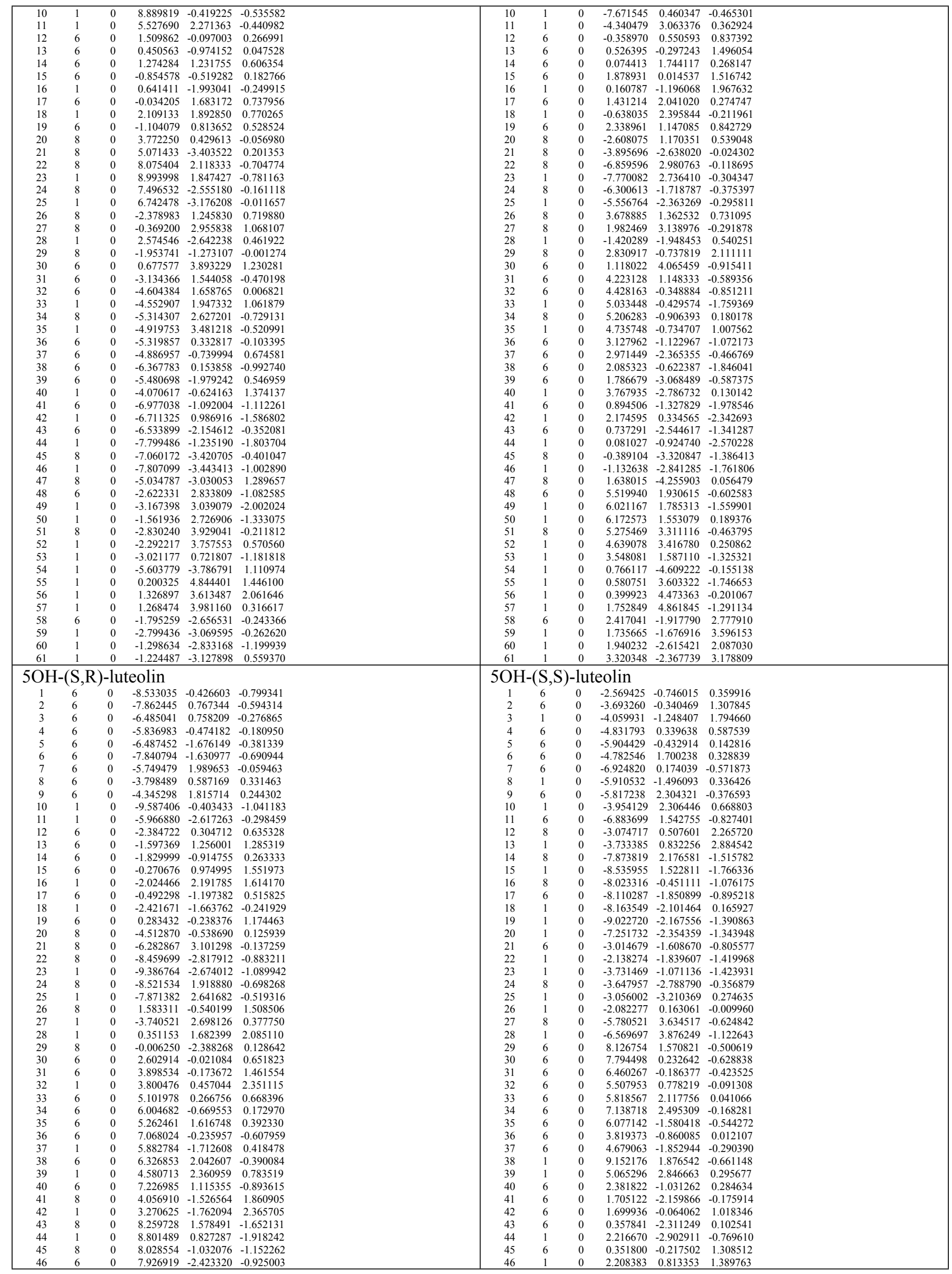




\begin{tabular}{|c|}
\hline 47 \\
\hline 48 \\
\hline 49 \\
\hline 50 \\
\hline 51 \\
\hline 52 \\
\hline 53 \\
\hline 54 \\
\hline 55 \\
\hline 56 \\
\hline 57 \\
\hline 58 \\
\hline $5 \mathrm{OH}$ \\
\hline 1 \\
\hline 2 \\
\hline 3 \\
\hline 4 \\
\hline 5 \\
\hline 6 \\
\hline 7 \\
\hline 8 \\
\hline 9 \\
\hline 10 \\
\hline 11 \\
\hline 12 \\
\hline 13 \\
\hline 14 \\
\hline 15 \\
\hline 16 \\
\hline 17 \\
\hline 18 \\
\hline 19 \\
\hline 20 \\
\hline 21 \\
\hline 22 \\
\hline 23 \\
\hline 24 \\
\hline 25 \\
\hline 26 \\
\hline 27 \\
\hline 28 \\
\hline 29 \\
\hline 30 \\
\hline 31 \\
\hline 32 \\
\hline 33 \\
\hline 34 \\
\hline 35 \\
\hline 36 \\
\hline 37 \\
\hline 38 \\
\hline 39 \\
\hline 40 \\
\hline 41 \\
\hline 42 \\
\hline 43 \\
\hline 44 \\
\hline 45 \\
\hline 46 \\
\hline 47 \\
\hline 48 \\
\hline 49 \\
\hline 50 \\
\hline 51 \\
\hline 52 \\
\hline 53 \\
\hline 54 \\
\hline 55 \\
\hline 56 \\
\hline 57 \\
\hline 58 \\
\hline 59 \\
\hline 60 \\
\hline 61 \\
\hline $5 \mathrm{OH}$ \\
\hline 1 \\
\hline 2 \\
\hline 3 \\
\hline 4 \\
\hline 5 \\
\hline 6 \\
\hline 7 \\
\hline 8 \\
\hline 9 \\
\hline 10 \\
\hline 11 \\
\hline 12 \\
\hline 13 \\
\hline 14 \\
\hline 15 \\
\hline 16 \\
\hline 17 \\
\hline 18 \\
\hline 19 \\
\hline 20 \\
\hline 21 \\
\hline 22 \\
\hline 23 \\
\hline
\end{tabular}




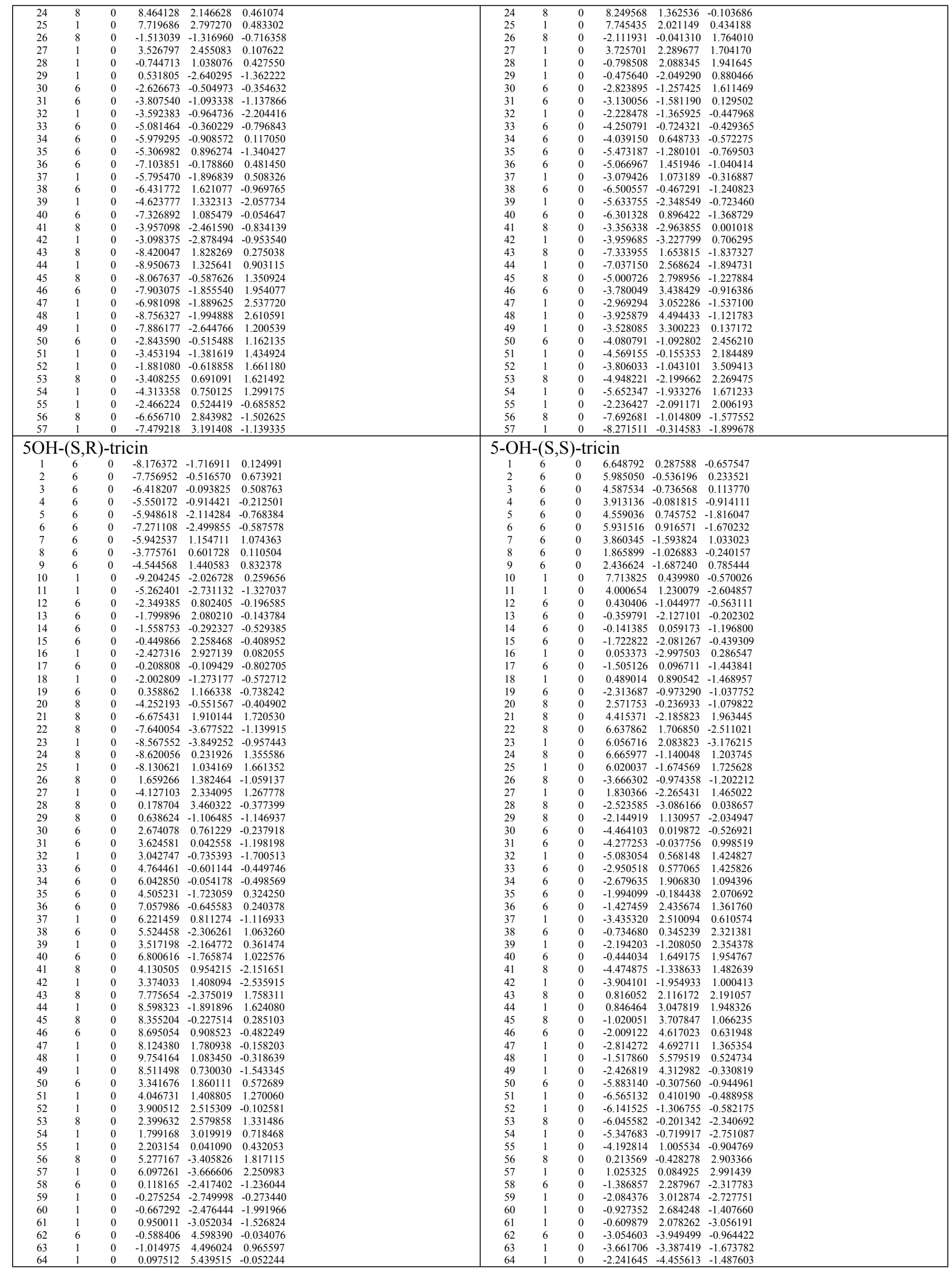




\begin{tabular}{|c|c|}
\hline 65 & 1 \\
\hline $\mathrm{G}-(\mathrm{S}$ & R)- \\
\hline 1 & 6 \\
\hline 2 & 6 \\
\hline 3 & 6 \\
\hline 4 & 6 \\
\hline 5 & 6 \\
\hline 6 & 6 \\
\hline 7 & 6 \\
\hline 8 & 6 \\
\hline 9 & 6 \\
\hline 10 & 1 \\
\hline 11 & 1 \\
\hline 12 & 6 \\
\hline 13 & 6 \\
\hline 14 & 6 \\
\hline 15 & 6 \\
\hline 16 & 1 \\
\hline 17 & 6 \\
\hline 18 & 1 \\
\hline 19 & 6 \\
\hline 20 & 8 \\
\hline 21 & 8 \\
\hline 22 & 8 \\
\hline 23 & 1 \\
\hline 24 & 8 \\
\hline 25 & 1 \\
\hline 26 & 8 \\
\hline 27 & 1 \\
\hline 28 & 1 \\
\hline 29 & 8 \\
\hline 30 & 6 \\
\hline 31 & 6 \\
\hline 32 & 1 \\
\hline 33 & 6 \\
\hline 34 & 6 \\
\hline 35 & 6 \\
\hline 36 & 6 \\
\hline 37 & 1 \\
\hline 38 & 6 \\
\hline 39 & 1 \\
\hline 40 & 6 \\
\hline 41 & 1 \\
\hline 42 & 8 \\
\hline 43 & 1 \\
\hline 44 & 8 \\
\hline 45 & 1 \\
\hline 46 & 8 \\
\hline 47 & 6 \\
\hline 48 & 1 \\
\hline 49 & 1 \\
\hline 50 & 1 \\
\hline 51 & 6 \\
\hline 52 & 1 \\
\hline 53 & 1 \\
\hline 54 & 8 \\
\hline 55 & 1 \\
\hline 56 & 1 \\
\hline 57 & 1 \\
\hline $\mathrm{G}-(\mathrm{S}, \mathrm{H}$ & -ch \\
\hline 1 & 6 \\
\hline 2 & 6 \\
\hline 3 & 6 \\
\hline 4 & 6 \\
\hline 5 & 6 \\
\hline 6 & 6 \\
\hline 7 & 6 \\
\hline 8 & 6 \\
\hline 9 & 6 \\
\hline 10 & 1 \\
\hline 11 & 1 \\
\hline 12 & 6 \\
\hline 13 & 6 \\
\hline 14 & 6 \\
\hline 15 & 6 \\
\hline 16 & 1 \\
\hline 17 & 6 \\
\hline 18 & 1 \\
\hline 19 & 6 \\
\hline 20 & 8 \\
\hline 21 & 8 \\
\hline 22 & 8 \\
\hline 23 & 1 \\
\hline 24 & 8 \\
\hline 25 & 1 \\
\hline 26 & 8 \\
\hline 27 & 1 \\
\hline 28 & 1 \\
\hline 29 & 8 \\
\hline 30 & 6 \\
\hline 31 & 6 \\
\hline 32 & 1 \\
\hline 33 & 6 \\
\hline 34 & 6 \\
\hline 35 & 6 \\
\hline 36 & 6 \\
\hline 37 & 1 \\
\hline
\end{tabular}




\begin{tabular}{|c|c|c|}
\hline 38 & 6 & 0 \\
\hline 39 & 1 & 0 \\
\hline 40 & 6 & 0 \\
\hline 41 & 1 & 0 \\
\hline 42 & 8 & 0 \\
\hline 43 & 1 & 0 \\
\hline 44 & 8 & 0 \\
\hline 45 & 1 & 0 \\
\hline 46 & 8 & 0 \\
\hline 47 & 6 & 0 \\
\hline 48 & 1 & 0 \\
\hline 49 & 1 & 0 \\
\hline 50 & 1 & 0 \\
\hline 51 & 6 & 0 \\
\hline 52 & 1 & 0 \\
\hline 53 & 1 & 0 \\
\hline 54 & 8 & 0 \\
\hline 55 & 1 & 0 \\
\hline 56 & 1 & 0 \\
\hline 57 & 6 & 0 \\
\hline 58 & 1 & 0 \\
\hline 59 & 1 & 0 \\
\hline 60 & 1 & 0 \\
\hline G-(S & 2)-a & gen \\
\hline 1 & 6 & 0 \\
\hline 2 & 6 & 0 \\
\hline 3 & 6 & 0 \\
\hline 4 & 6 & 0 \\
\hline 5 & 6 & 0 \\
\hline 6 & 6 & 0 \\
\hline 7 & 6 & 0 \\
\hline 8 & 6 & 0 \\
\hline 9 & 6 & 0 \\
\hline 10 & 1 & 0 \\
\hline 11 & 1 & 0 \\
\hline 12 & 6 & 0 \\
\hline 13 & 6 & 0 \\
\hline 14 & 6 & 0 \\
\hline 15 & 6 & 0 \\
\hline 16 & 1 & 0 \\
\hline 17 & 6 & 0 \\
\hline 18 & 1 & 0 \\
\hline 19 & 6 & 0 \\
\hline 20 & 8 & 0 \\
\hline 21 & 8 & 0 \\
\hline 22 & 8 & 0 \\
\hline 23 & 1 & 0 \\
\hline 24 & 8 & 0 \\
\hline 25 & 1 & 0 \\
\hline 26 & 8 & 0 \\
\hline 27 & 1 & 0 \\
\hline 28 & 1 & 0 \\
\hline 29 & 1 & 0 \\
\hline 30 & 6 & 0 \\
\hline 31 & 6 & 0 \\
\hline 32 & 1 & 0 \\
\hline 33 & 6 & 0 \\
\hline 34 & 6 & 0 \\
\hline 35 & 6 & 0 \\
\hline 36 & 6 & 0 \\
\hline 37 & 1 & 0 \\
\hline 38 & 6 & 0 \\
\hline 39 & 1 & 0 \\
\hline 40 & 6 & 0 \\
\hline 41 & 1 & 0 \\
\hline 42 & 8 & 0 \\
\hline 43 & 1 & 0 \\
\hline 44 & 8 & 0 \\
\hline 45 & 1 & 0 \\
\hline 46 & 8 & 0 \\
\hline 47 & 6 & 0 \\
\hline 48 & 1 & 0 \\
\hline 49 & 1 & 0 \\
\hline 50 & 1 & 0 \\
\hline 51 & 6 & 0 \\
\hline 52 & 1 & 0 \\
\hline 53 & 1 & 0 \\
\hline 54 & 8 & 0 \\
\hline 55 & 1 & 0 \\
\hline 56 & 1 & 0 \\
\hline G-(S & R)- $t$ & \\
\hline 1 & 6 & 0 \\
\hline 2 & 6 & 0 \\
\hline 3 & 6 & 0 \\
\hline 4 & 6 & 0 \\
\hline 5 & 6 & 0 \\
\hline 6 & 6 & 0 \\
\hline 7 & 6 & 0 \\
\hline 8 & 6 & 0 \\
\hline 9 & 6 & 0 \\
\hline 10 & 1 & 0 \\
\hline 11 & 1 & 0 \\
\hline 12 & 6 & 0 \\
\hline 13 & 6 & 0 \\
\hline 14 & 6 & 0 \\
\hline 15 & 6 & 0 \\
\hline
\end{tabular}




\begin{tabular}{|c|c|}
\hline 16 & 1 \\
\hline 17 & 6 \\
\hline 18 & 1 \\
\hline 19 & 6 \\
\hline 20 & 8 \\
\hline 21 & 8 \\
\hline 22 & 8 \\
\hline 23 & 1 \\
\hline 24 & 8 \\
\hline 25 & 1 \\
\hline 26 & 8 \\
\hline 27 & 1 \\
\hline 28 & 8 \\
\hline 29 & 8 \\
\hline 30 & 6 \\
\hline 31 & 6 \\
\hline 32 & 1 \\
\hline 33 & 6 \\
\hline 34 & 6 \\
\hline 35 & 6 \\
\hline 36 & 6 \\
\hline 37 & 1 \\
\hline 38 & 6 \\
\hline 39 & 1 \\
\hline 40 & 6 \\
\hline 41 & 1 \\
\hline 42 & 8 \\
\hline 43 & 1 \\
\hline 44 & 8 \\
\hline 45 & 1 \\
\hline 46 & 8 \\
\hline 47 & 6 \\
\hline 48 & 1 \\
\hline 49 & 1 \\
\hline 50 & 1 \\
\hline 51 & 6 \\
\hline 52 & 1 \\
\hline 53 & 1 \\
\hline 54 & 8 \\
\hline 55 & 1 \\
\hline 56 & 1 \\
\hline 57 & 6 \\
\hline 58 & 1 \\
\hline 59 & 1 \\
\hline 60 & 1 \\
\hline 61 & 6 \\
\hline 62 & 1 \\
\hline 63 & 1 \\
\hline 64 & 1 \\
\hline Fs-( & R)- \\
\hline 1 & 6 \\
\hline 2 & 6 \\
\hline 3 & 6 \\
\hline 4 & 6 \\
\hline 5 & 6 \\
\hline 6 & 6 \\
\hline 7 & 6 \\
\hline 8 & 6 \\
\hline 9 & 6 \\
\hline 10 & 1 \\
\hline 11 & 1 \\
\hline 12 & 6 \\
\hline 13 & 6 \\
\hline 14 & 6 \\
\hline 15 & 6 \\
\hline 16 & 1 \\
\hline 17 & 6 \\
\hline 18 & 1 \\
\hline 19 & 6 \\
\hline 20 & 8 \\
\hline 21 & 8 \\
\hline 22 & 8 \\
\hline 23 & 1 \\
\hline 24 & 8 \\
\hline 25 & 1 \\
\hline 26 & 8 \\
\hline 27 & 1 \\
\hline 28 & 1 \\
\hline 29 & 8 \\
\hline 30 & 6 \\
\hline 31 & 1 \\
\hline 32 & 6 \\
\hline 33 & 1 \\
\hline 34 & 6 \\
\hline 35 & 6 \\
\hline 36 & 6 \\
\hline 37 & 6 \\
\hline 38 & 1 \\
\hline 39 & 6 \\
\hline 40 & 1 \\
\hline 41 & 6 \\
\hline 42 & 1 \\
\hline 43 & 8 \\
\hline 44 & 8 \\
\hline 45 & 1 \\
\hline 46 & 8 \\
\hline 47 & 1 \\
\hline
\end{tabular}




\begin{tabular}{|c|c|}
\hline & \\
\hline 48 & 8 \\
\hline 49 & 6 \\
\hline 50 & 1 \\
\hline 51 & 1 \\
\hline 52 & 1 \\
\hline 53 & 6 \\
\hline 54 & 6 \\
\hline 55 & 1 \\
\hline 56 & 1 \\
\hline 57 & 6 \\
\hline 58 & 1 \\
\hline 59 & 1 \\
\hline 60 & 1 \\
\hline 61 & 8 \\
\hline 62 & 1 \\
\hline Fs-(S & ) $-\mathrm{c}$ \\
\hline 1 & 6 \\
\hline 2 & 6 \\
\hline 3 & 6 \\
\hline 4 & 6 \\
\hline 5 & 6 \\
\hline 6 & 6 \\
\hline 7 & 6 \\
\hline 8 & 6 \\
\hline 9 & 6 \\
\hline 10 & 1 \\
\hline 11 & 1 \\
\hline 12 & 6 \\
\hline 13 & 6 \\
\hline 14 & 6 \\
\hline 15 & 6 \\
\hline 16 & 1 \\
\hline 17 & 6 \\
\hline 18 & 1 \\
\hline 19 & 6 \\
\hline 20 & 8 \\
\hline 21 & 8 \\
\hline 22 & 8 \\
\hline 23 & 1 \\
\hline 24 & 8 \\
\hline 25 & 1 \\
\hline 26 & 8 \\
\hline 27 & 1 \\
\hline 28 & 1 \\
\hline 29 & 8 \\
\hline 30 & 6 \\
\hline 31 & 1 \\
\hline 32 & 6 \\
\hline 33 & 1 \\
\hline 34 & 6 \\
\hline 35 & 6 \\
\hline 36 & 6 \\
\hline 37 & 6 \\
\hline 38 & 1 \\
\hline 39 & 6 \\
\hline 40 & 1 \\
\hline 41 & 6 \\
\hline 42 & 1 \\
\hline 43 & 8 \\
\hline 44 & 8 \\
\hline 45 & 1 \\
\hline 46 & 8 \\
\hline 47 & 1 \\
\hline 48 & 8 \\
\hline 49 & 6 \\
\hline 50 & 1 \\
\hline 51 & 1 \\
\hline 52 & 1 \\
\hline 53 & 6 \\
\hline 54 & 6 \\
\hline 55 & 1 \\
\hline 56 & 1 \\
\hline 57 & 6 \\
\hline 58 & 1 \\
\hline 59 & 1 \\
\hline 60 & 1 \\
\hline 61 & 8 \\
\hline 62 & 6 \\
\hline 63 & 1 \\
\hline 64 & 1 \\
\hline 65 & 1 \\
\hline Fs- $(S$ & R)- \\
\hline 1 & 6 \\
\hline 2 & 6 \\
\hline 3 & 6 \\
\hline 4 & 6 \\
\hline 5 & 6 \\
\hline 6 & 6 \\
\hline 7 & 6 \\
\hline 8 & 6 \\
\hline 9 & 6 \\
\hline 10 & 1 \\
\hline 11 & 1 \\
\hline 12 & 6 \\
\hline 13 & 6 \\
\hline 14 & 6 \\
\hline
\end{tabular}




\begin{tabular}{|c|}
\hline 15 \\
\hline 16 \\
\hline 17 \\
\hline 18 \\
\hline 19 \\
\hline 20 \\
\hline 21 \\
\hline 22 \\
\hline 23 \\
\hline 24 \\
\hline 25 \\
\hline 26 \\
\hline 27 \\
\hline 28 \\
\hline 29 \\
\hline 30 \\
\hline 31 \\
\hline 32 \\
\hline 33 \\
\hline 34 \\
\hline 35 \\
\hline 36 \\
\hline 37 \\
\hline 38 \\
\hline 39 \\
\hline 40 \\
\hline 41 \\
\hline 42 \\
\hline 43 \\
\hline 44 \\
\hline 45 \\
\hline 46 \\
\hline 47 \\
\hline 48 \\
\hline 49 \\
\hline 50 \\
\hline 51 \\
\hline 52 \\
\hline 53 \\
\hline 54 \\
\hline 55 \\
\hline 56 \\
\hline 57 \\
\hline 58 \\
\hline 59 \\
\hline 60 \\
\hline 61 \\
\hline Fs-(S,R \\
\hline 1 \\
\hline 2 \\
\hline 3 \\
\hline 4 \\
\hline 5 \\
\hline 6 \\
\hline 7 \\
\hline 8 \\
\hline 9 \\
\hline 10 \\
\hline 11 \\
\hline 12 \\
\hline 13 \\
\hline 14 \\
\hline 15 \\
\hline 16 \\
\hline 17 \\
\hline 18 \\
\hline 19 \\
\hline 20 \\
\hline 21 \\
\hline 22 \\
\hline 23 \\
\hline 24 \\
\hline 25 \\
\hline 26 \\
\hline 27 \\
\hline 28 \\
\hline 29 \\
\hline 30 \\
\hline 31 \\
\hline 32 \\
\hline 33 \\
\hline 34 \\
\hline 35 \\
\hline 36 \\
\hline 37 \\
\hline 38 \\
\hline 39 \\
\hline 40 \\
\hline 41 \\
\hline 42 \\
\hline 43 \\
\hline 44 \\
\hline 45 \\
\hline 46 \\
\hline 47 \\
\hline 48 \\
\hline 49 \\
\hline 50 \\
\hline
\end{tabular}




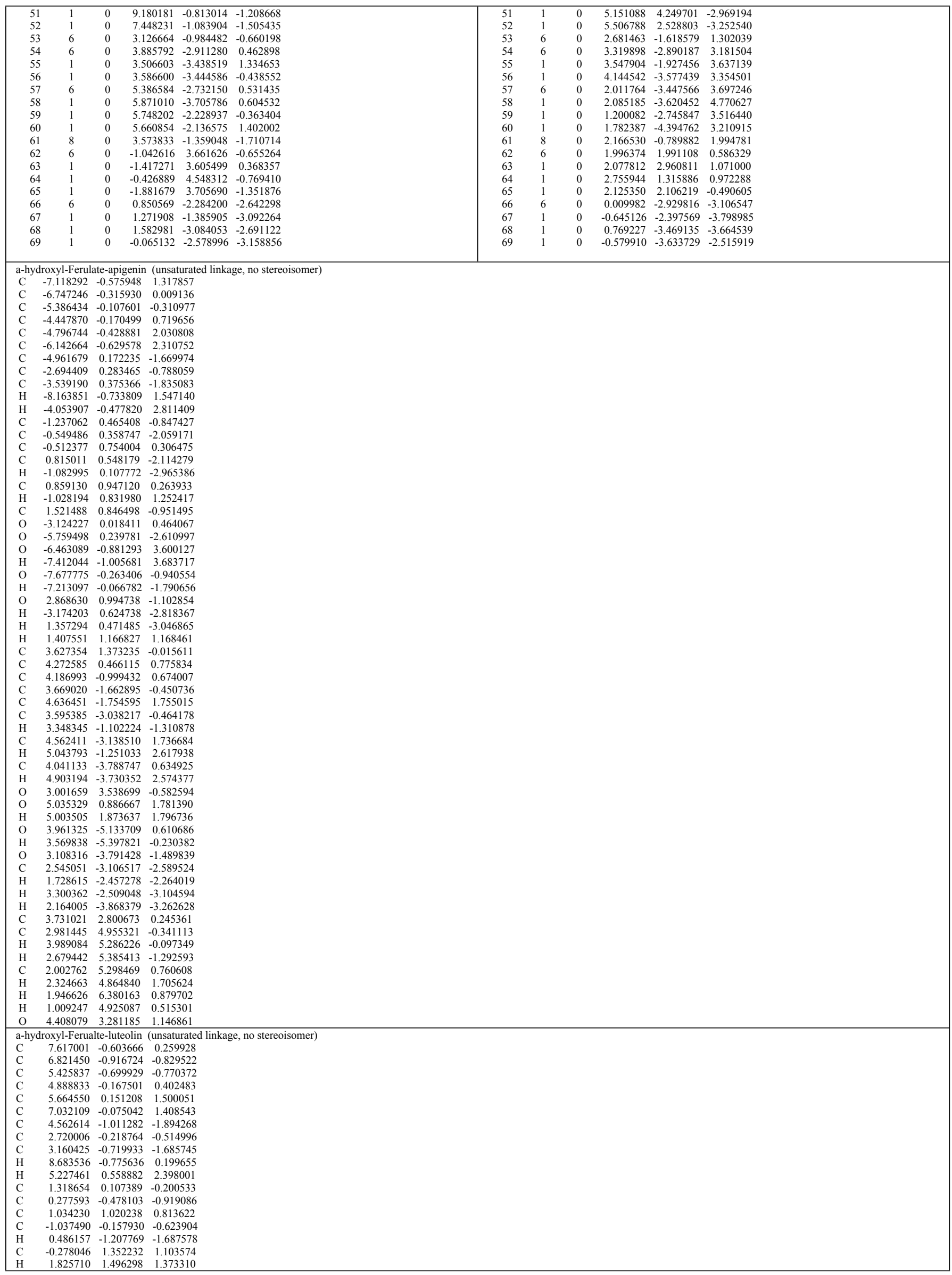




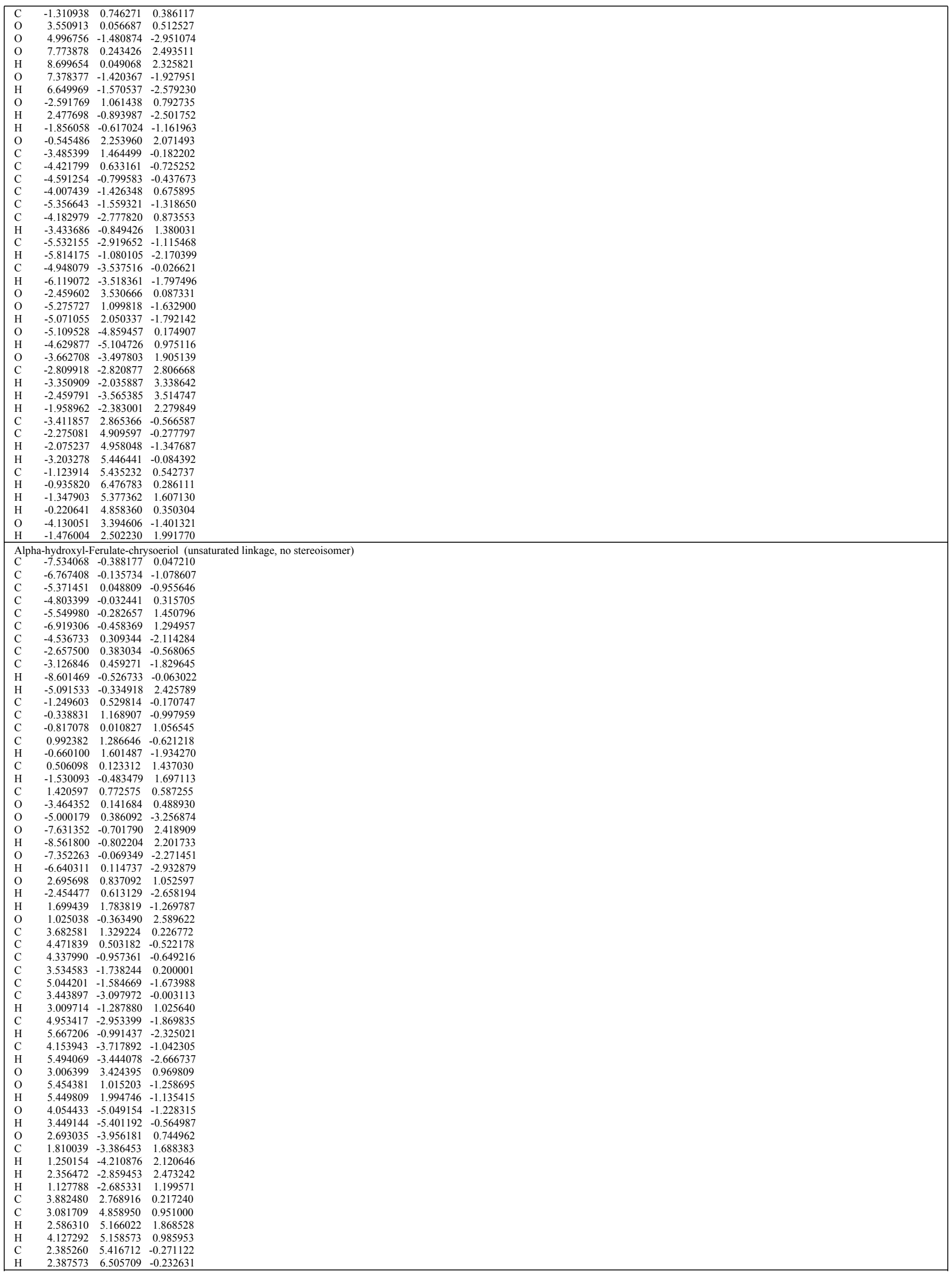




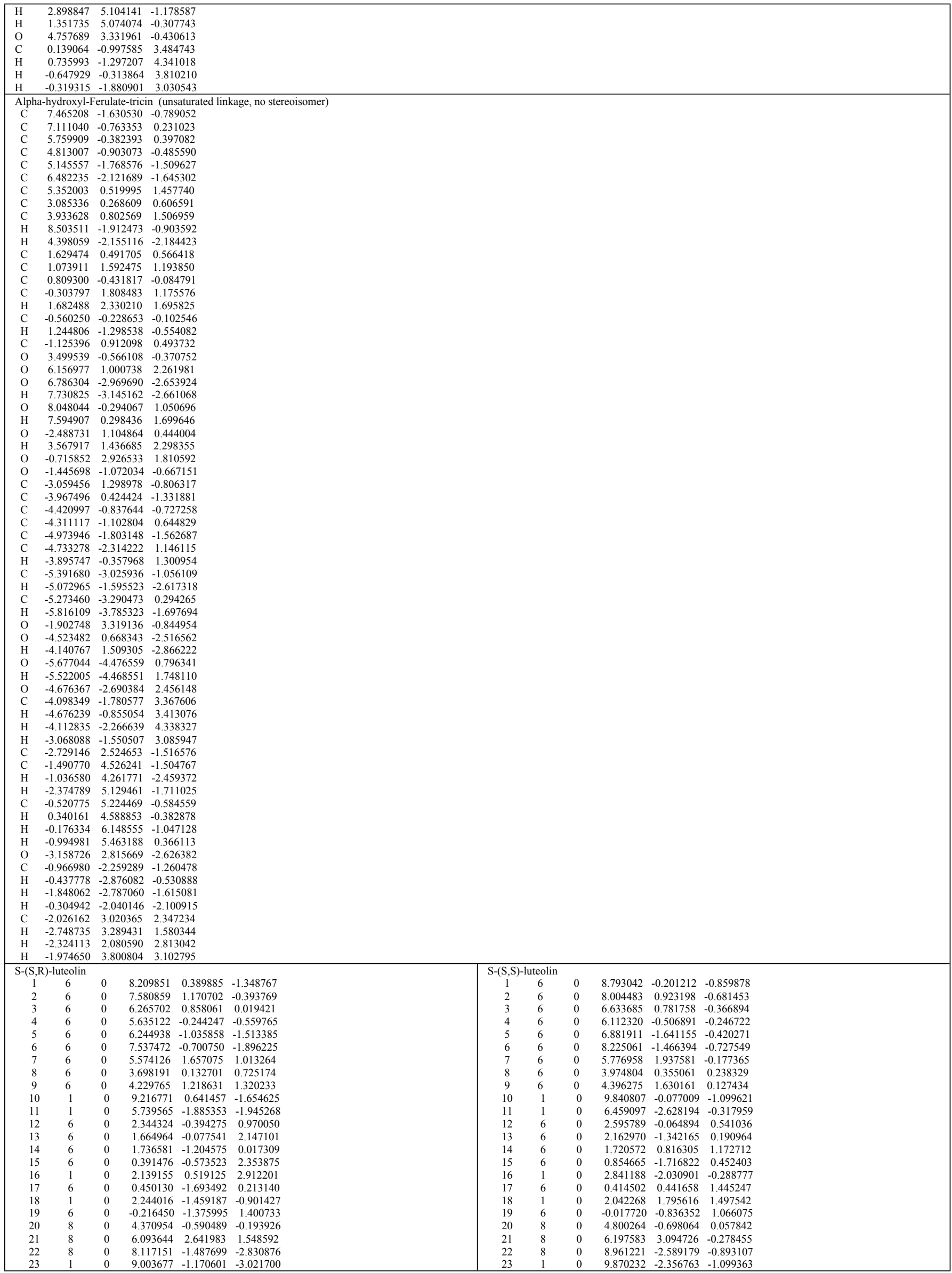




\begin{tabular}{|c|}
\hline 24 \\
\hline 25 \\
\hline 26 \\
\hline 27 \\
\hline 28 \\
\hline 29 \\
\hline 30 \\
\hline 31 \\
\hline 32 \\
\hline 33 \\
\hline 34 \\
\hline 35 \\
\hline 36 \\
\hline 37 \\
\hline 38 \\
\hline 39 \\
\hline 40 \\
\hline 41 \\
\hline 42 \\
\hline 43 \\
\hline 44 \\
\hline 45 \\
\hline 46 \\
\hline 47 \\
\hline 48 \\
\hline 49 \\
\hline 50 \\
\hline 51 \\
\hline 52 \\
\hline 53 \\
\hline 54 \\
\hline 55 \\
\hline 56 \\
\hline 57 \\
\hline 58 \\
\hline 59 \\
\hline 60 \\
\hline 61 \\
\hline S-(S,R) \\
\hline 1 \\
\hline 2 \\
\hline 3 \\
\hline 4 \\
\hline 5 \\
\hline 6 \\
\hline 7 \\
\hline 8 \\
\hline 9 \\
\hline 10 \\
\hline 11 \\
\hline 12 \\
\hline 13 \\
\hline 14 \\
\hline 15 \\
\hline 16 \\
\hline 17 \\
\hline 18 \\
\hline 19 \\
\hline 20 \\
\hline 21 \\
\hline 22 \\
\hline 23 \\
\hline 24 \\
\hline 25 \\
\hline 26 \\
\hline 27 \\
\hline 28 \\
\hline 29 \\
\hline 30 \\
\hline 31 \\
\hline 32 \\
\hline 33 \\
\hline 34 \\
\hline 35 \\
\hline 36 \\
\hline 37 \\
\hline 38 \\
\hline 39 \\
\hline 40 \\
\hline 41 \\
\hline 42 \\
\hline 43 \\
\hline 44 \\
\hline 45 \\
\hline 46 \\
\hline 47 \\
\hline 48 \\
\hline 49 \\
\hline 50 \\
\hline 51 \\
\hline 52 \\
\hline 53 \\
\hline 54 \\
\hline 55 \\
\hline 56 \\
\hline 57 \\
\hline 58 \\
\hline 59 \\
\hline
\end{tabular}




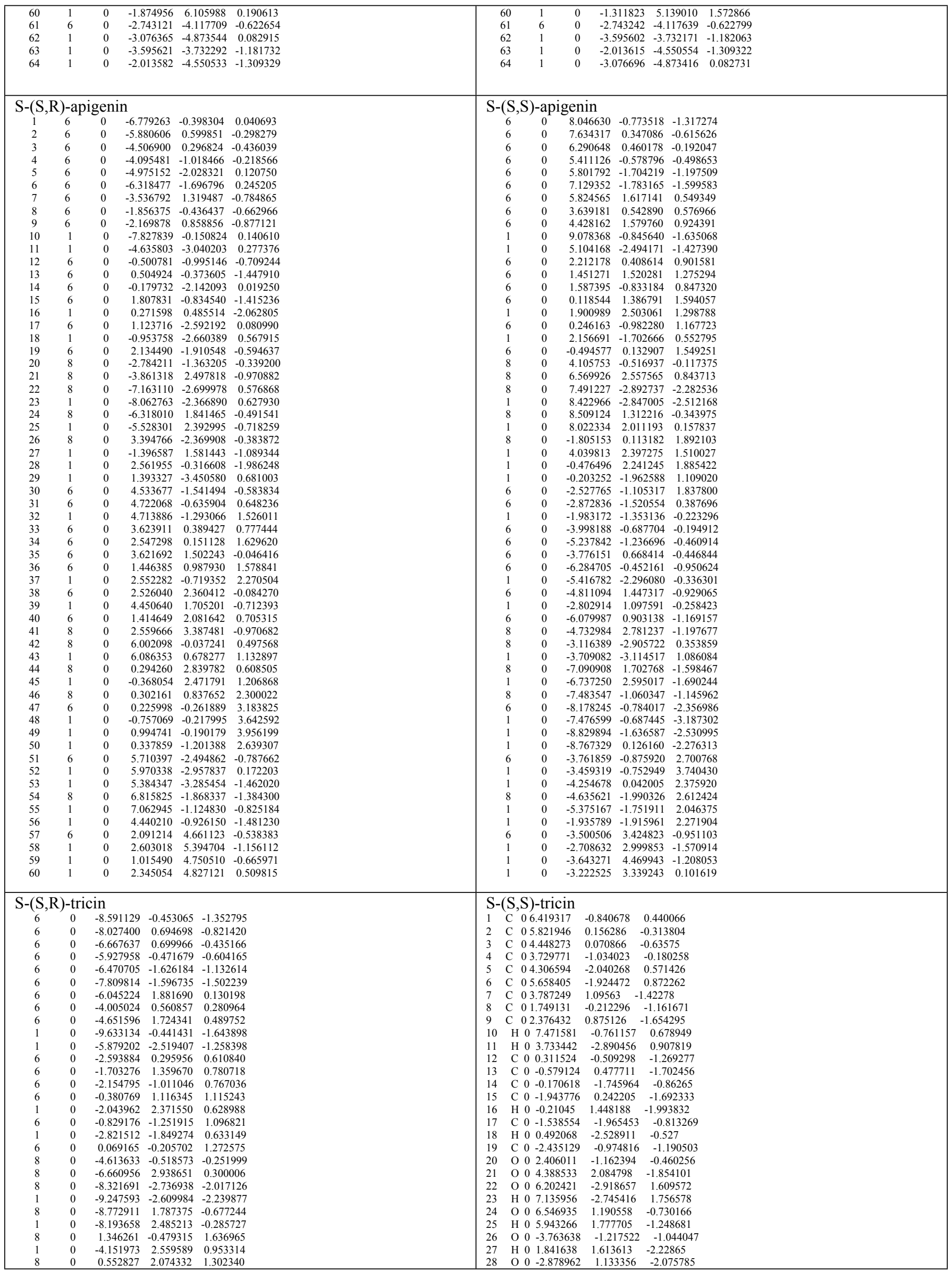




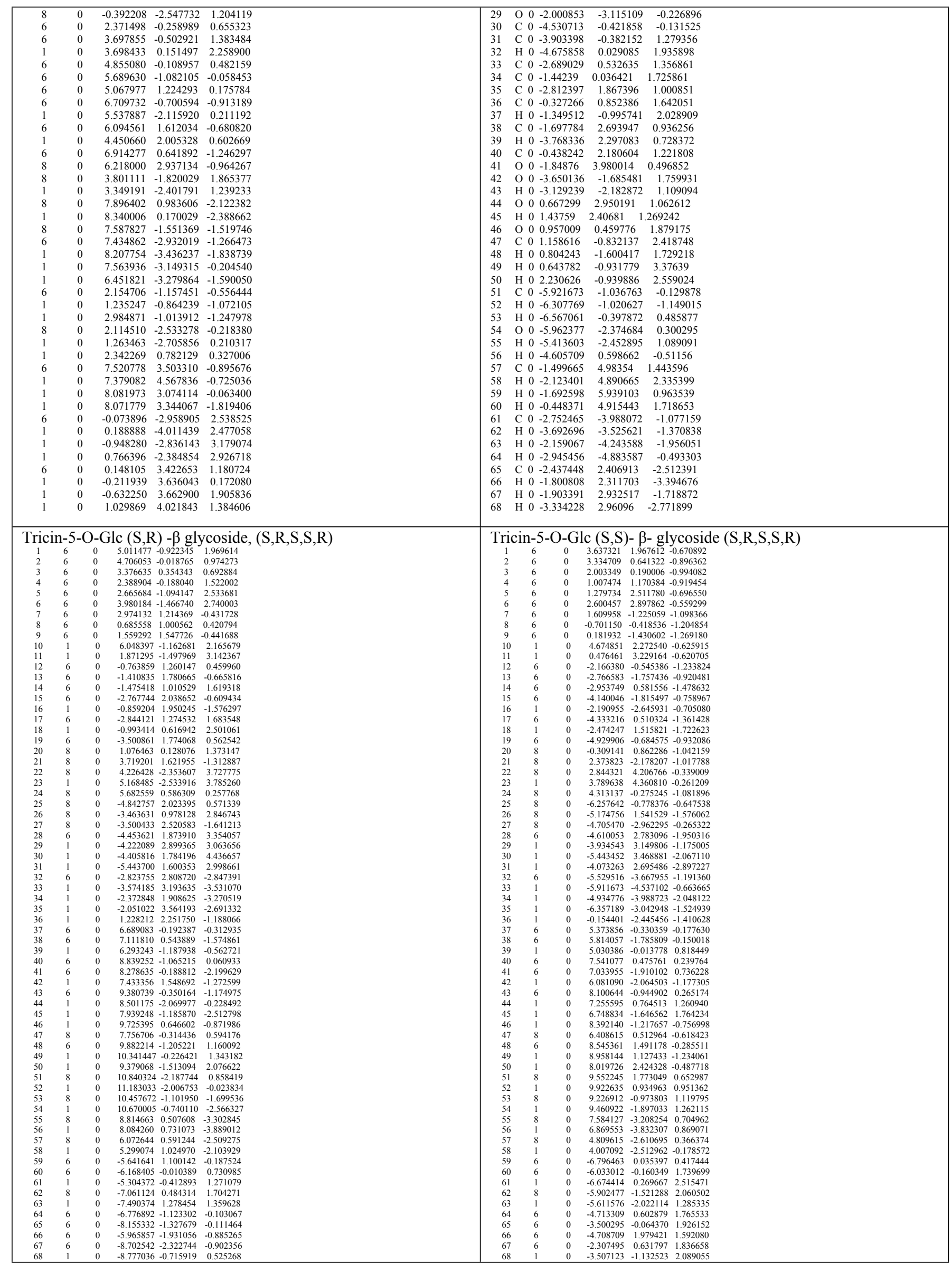




\begin{tabular}{|c|c|c|c|c|c|c|c|c|c|c|c|}
\hline $\begin{array}{l}69 \\
70 \\
71 \\
72 \\
73 \\
74 \\
75 \\
76 \\
77 \\
78 \\
79 \\
80 \\
81 \\
82 \\
83 \\
84 \\
85\end{array}$ & $\begin{array}{l}6 \\
1 \\
6 \\
1 \\
8 \\
1 \\
8 \\
6 \\
1 \\
1 \\
1 \\
6 \\
1 \\
1 \\
8 \\
1 \\
1\end{array}$ & $\begin{array}{l}0 \\
0 \\
0 \\
0 \\
0 \\
0 \\
0 \\
0 \\
0 \\
0 \\
0 \\
0 \\
0 \\
0 \\
0 \\
0 \\
0\end{array}$ & 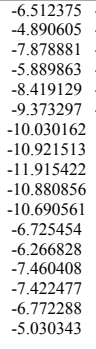 & $\begin{array}{l}-2.930944 \\
-1.795023 \\
-3.129056 \\
-3.5711938 \\
-4.103982 \\
-4.096241 \\
-2.6222744 \\
-1.867195 \\
-2.255772 \\
-0.809589 \\
-1.979077 \\
1.973032 \\
2.564072 \\
1.280879 \\
2.728050 \\
3.305349 \\
0.643680\end{array}$ & $\begin{array}{c}-1.681314 \\
-0.867623 \\
-1.697748 \\
-2.290030 \\
-2.467772 \\
-2.333902 \\
-0.990091 \\
-0.198452 \\
-0.3987767 \\
-0.467594 \\
0.862751 \\
-0.851259 \\
-1.614584 \\
-1.322100 \\
0.098448 \\
0.512731 \\
-0.967426\end{array}$ & $\begin{array}{l}69 \\
70 \\
71 \\
72 \\
73 \\
74 \\
75 \\
76 \\
77 \\
78 \\
79 \\
80 \\
81 \\
82 \\
83 \\
84 \\
85\end{array}$ & $\begin{array}{l}6 \\
1 \\
6 \\
1 \\
8 \\
1 \\
8 \\
6 \\
1 \\
1 \\
1 \\
6 \\
1 \\
1 \\
8 \\
1 \\
1\end{array}$ & $\begin{array}{l}0 \\
0 \\
0 \\
0 \\
0 \\
0 \\
0 \\
0 \\
0 \\
0 \\
0 \\
0 \\
0 \\
0 \\
0 \\
0 \\
0\end{array}$ & $\begin{array}{l}-3.513381 \\
-5.6438379 \\
-2.309341 \\
-3.498557 \\
-1.140082 \\
-0.415318 \\
-1.060688 \\
-0.964457 \\
-1.4185513 \\
0.095560 \\
-1.451127 \\
-8.241138 \\
-8.270778 \\
-8.7435511 \\
-8.934996 \\
-8.410975 \\
-6.756806\end{array}$ & $\begin{array}{l}2.6855977 \\
2.517822 \\
2.015352 \\
3.759722 \\
2.694497 \\
2.063628 \\
0.081603 \\
-1.289752 \\
-1.485903 \\
-1.529257 \\
-1.905813 \\
-0.411122 \\
-1.463542 \\
0.177719 \\
-0.193707 \\
-0.578232 \\
1.081292\end{array}$ & $\begin{array}{l}.527383 \\
1.495029 \\
1.623872 \\
1.399013 \\
1.516687 \\
1.606758 \\
1.906713 \\
2.235539 \\
3.208913 \\
2.268617 \\
1.476913 \\
0.518661 \\
0.817762 \\
1.285931 \\
-0.687690 \\
-1.396044 \\
0.113309\end{array}$ \\
\hline & & & & & & & & & & & \\
\hline Trici & $1-6$ & Ara & $8 \mathrm{Glc}(\mathrm{s}$ & S,R) & & Tric & $1-6$ & Ara & 8 Glc & S,S) & \\
\hline 1 & & & & & & & & & & & \\
\hline $\begin{array}{l}2 \\
3\end{array}$ & 6 & $0_{0}$ & $\begin{array}{l}3.248937 \\
1881917\end{array}$ & $\begin{array}{l}-1.9773322 \\
1.68589\end{array}$ & 0.359703 & 2 & 6 & 0 & $\begin{array}{l}3.216231 \\
1810979\end{array}$ & $\begin{array}{l}-2.143677 \\
-2\end{array}$ & $\begin{array}{r}-0.732645 \\
\end{array}$ \\
\hline 4 & $\begin{array}{l}0 \\
6\end{array}$ & 0 & $\begin{array}{l}1.8191917 \\
1.444944\end{array}$ & $\begin{array}{l}-1.66588888 \\
-0.377959\end{array}$ & $\begin{array}{l}0.4935516 \\
0.154740\end{array}$ & $\begin{array}{l}3 \\
4\end{array}$ & $\begin{array}{l}6 \\
6\end{array}$ & $\begin{array}{l}0 \\
0\end{array}$ & $\begin{array}{l}1.819877 \\
1.222953\end{array}$ & $\begin{array}{l}-2.00889997 \\
-0.817943\end{array}$ & $\begin{array}{l}-0.877650 \\
-0.992019\end{array}$ \\
\hline 5 & 6 & 0 & 2.327369 & 0.600111 & -0.283766 & 5 & 6 & 0 & 1.955834 & 0.361368 & -0.947274 \\
\hline 6 & 6 & 0 & $\begin{array}{l}3.672922 \\
0.92012\end{array}$ & 0.265911 & $\begin{array}{l}-0.430517 \\
0.06907\end{array}$ & 6 & 6 & 0 & $\begin{array}{l}3.337704 \\
0.9007\end{array}$ & 0.275670 & $\begin{array}{l}-0.793105 \\
\end{array}$ \\
\hline $\begin{array}{l}7 \\
8\end{array}$ & ${ }_{6}^{6}$ & $\begin{array}{l}0 \\
0\end{array}$ & $\begin{array}{r}0.932947 \\
-0.72382\end{array}$ & $\begin{array}{l}-2.644900 \\
-0.940770\end{array}$ & $\begin{array}{l}0.986972 \\
0.667546\end{array}$ & $\begin{array}{l}7 \\
8\end{array}$ & $\begin{array}{l}6 \\
6\end{array}$ & $\begin{array}{l}0 \\
0 \\
0\end{array}$ & $\begin{array}{l}0.992070 \\
-0.923303\end{array}$ & $\begin{array}{r}-3.264155 \\
-176000\end{array}$ & $\begin{array}{l}-0.9111222 \\
-1133911\end{array}$ \\
\hline 9 & $\begin{array}{l}0 \\
6\end{array}$ & 0 & $\begin{array}{l}-0.7272382 \\
-0.435142\end{array}$ & $\begin{array}{l}-0.9447770 \\
-2.191208\end{array}$ & $\begin{array}{l}0.6676466 \\
1.055708\end{array}$ & $\begin{array}{l}8 \\
9\end{array}$ & $\begin{array}{l}6 \\
6\end{array}$ & $\begin{array}{l}0 \\
0 \\
0\end{array}$ & $\begin{array}{l}-0.923303 \\
-0.425948\end{array}$ & $\begin{array}{l}-1.768004 \\
-3.021571\end{array}$ & $\begin{array}{l}-1.1339318 \\
-1.045766\end{array}$ \\
\hline 10 & 6 & 0 & -2.146677 & -0.423769 & 0.646702 & 10 & 6 & 0 & $\begin{array}{l}-2.343015 \\
-24015\end{array}$ & -1.390874 & $\begin{array}{l}-1.201961 \\
\end{array}$ \\
\hline 11 & 6 & 0 & -3.060076 & -0.821667 & 1.625454 & 11 & 6 & 0 & $\begin{array}{r}-3.327350 \\
\end{array}$ & -2.372761 & -1.185363 \\
\hline 12 & $\begin{array}{l}6 \\
6\end{array}$ & $\begin{array}{l}0 \\
0 \\
0\end{array}$ & $\begin{array}{l}-2.542848 \\
-4.377978\end{array}$ & $\begin{array}{c}0.418015 \\
-0.39665\end{array}$ & $\begin{array}{r}-0.380654 \\
1558262\end{array}$ & ${ }_{12}^{12}$ & $\begin{array}{l}6 \\
6\end{array}$ & $\begin{array}{l}0 \\
0\end{array}$ & $\begin{array}{r}-2.6999523 \\
-6557936\end{array}$ & $\begin{array}{l}-0.037157 \\
-2004774\end{array}$ & $\begin{array}{l}-1.211682 \\
-1093191\end{array}$ \\
\hline $\begin{array}{l}13 \\
14\end{array}$ & $\begin{array}{l}6 \\
1\end{array}$ & $\begin{array}{l}0 \\
0\end{array}$ & $\begin{array}{l}-4.3779755 \\
-2.727845\end{array}$ & $\begin{array}{r}-0.396651 \\
-1.453831\end{array}$ & $\begin{array}{l}1.558262 \\
2.434726\end{array}$ & $\begin{array}{l}13 \\
14\end{array}$ & $\begin{array}{l}6 \\
1\end{array}$ & $\begin{array}{l}0 \\
0 \\
0\end{array}$ & $\begin{array}{l}-4.657936 \\
-3.096300\end{array}$ & $\begin{array}{l}-2.004774 \\
-3.427627\end{array}$ & $\begin{array}{l}-1.093191 \\
-1.18269\end{array}$ \\
\hline 15 & 6 & 0 & -3.870059 & $\begin{array}{r}-1.435831 \\
0.815814\end{array}$ & $\begin{array}{r}2.436720 \\
-0.467356\end{array}$ & $\begin{array}{l}14 \\
15\end{array}$ & 6 & $\begin{array}{l}0 \\
0\end{array}$ & $\begin{array}{r}-3.0960000 \\
-4.038477\end{array}$ & $\begin{array}{c}-3.42627 \gamma \\
0.326502\end{array}$ & $\begin{array}{l}-1.188629 \\
-1.176775\end{array}$ \\
\hline $\begin{array}{l}16 \\
11\end{array}$ & $\frac{1}{6}$ & $\begin{array}{l}0 \\
0\end{array}$ & $\begin{array}{r}-1.847988 \\
-4803677\end{array}$ & $\begin{array}{l}0.7631111 \\
0.388932\end{array}$ & $\begin{array}{r}-1.132940 \\
0477855\end{array}$ & 16 & 1 & 0 & $\begin{array}{r}-1.937312 \\
-50244\end{array}$ & 0.729102 & $\begin{array}{r}-1.221976 \\
1.055704\end{array}$ \\
\hline $\begin{array}{l}17 \\
18\end{array}$ & $\begin{array}{l}6 \\
8\end{array}$ & $\begin{array}{l}0 \\
0\end{array}$ & $\begin{array}{r}-4.8036777 \\
0.13551\end{array}$ & $\begin{array}{r}0.389832 \\
-0.036641\end{array}$ & $\begin{array}{l}0.472857 \\
0.238725\end{array}$ & $\begin{array}{l}17 \\
18\end{array}$ & $\begin{array}{l}6 \\
8\end{array}$ & $\begin{array}{l}0 \\
0\end{array}$ & $\begin{array}{l}-5.0244411 \\
-0.116334\end{array}$ & $\begin{array}{l}-0.6643066 \\
-0.685525\end{array}$ & $\begin{array}{l}-1.055794 \\
-1.139912\end{array}$ \\
\hline 19 & 8 & 0 & 1.289074 & $\begin{array}{l}-0.0050641 \\
-3.786290\end{array}$ & 1.299095 & $\begin{array}{l}18 \\
19\end{array}$ & 8 & $\begin{array}{l}0 \\
0\end{array}$ & $\begin{array}{r}-0.1166354 \\
1.480737\end{array}$ & $\begin{array}{l}-0.684525 \\
-4.393647\end{array}$ & $\begin{array}{l}-1.139912 \\
-0.811297\end{array}$ \\
\hline 20 & 8 & 0 & $\begin{array}{l}4.516351 \\
5.1371\end{array}$ & 1.217009 & -0.872388 & 20 & 8 & 0 & 4.036392 & 1.421065 & -0.748339 \\
\hline $\begin{array}{l}21 \\
22\end{array}$ & $\begin{array}{l}1 \\
8\end{array}$ & $\begin{array}{l}0 \\
0\end{array}$ & $\begin{array}{l}5.441371 \\
3.713393\end{array}$ & $\begin{array}{c}0.927902 \\
-3.18938\end{array}$ & $\begin{array}{c}-0.769874 \\
0.678953\end{array}$ & 21 & $\begin{array}{l}1 \\
8\end{array}$ & 0 & $\begin{array}{l}4.965815 \\
3847020\end{array}$ & $\begin{array}{l}1.255629 \\
-3.317775\end{array}$ & $\begin{array}{r}-0.504651 \\
\end{array}$ \\
\hline 23 & $\begin{array}{l}0 \\
1\end{array}$ & 0 & 2.930178 & $\begin{array}{l}-3.1883581 \\
-3.714773\end{array}$ & $\begin{array}{l}0.6878953 \\
0.994329\end{array}$ & 23 & $\begin{array}{l}8 \\
1\end{array}$ & $\begin{array}{l}0 \\
0\end{array}$ & $\begin{array}{l}3.84 / 720 \\
3.150908\end{array}$ & $\begin{array}{l}-3.31 \\
-4.018683\end{array}$ & $\begin{array}{l}-0.635918 \\
-0.675580\end{array}$ \\
\hline 24 & 8 & 0 & -6.118835 & 0.723424 & 0.393875 & 24 & 8 & 0 & -6.336058 & $\begin{array}{r}-0.360000 \\
\end{array}$ & -0.884204 \\
\hline $\begin{array}{l}25 \\
26\end{array}$ & $\begin{array}{l}8 \\
8\end{array}$ & $\begin{array}{l}0 \\
0\end{array}$ & $\begin{array}{l}-4.288008 \\
-53227089\end{array}$ & $\begin{array}{r}1.568247 \\
-0.72765\end{array}$ & $\begin{array}{r}-1.527725 \\
2464247\end{array}$ & $\begin{array}{l}25 \\
26\end{array}$ & $\begin{array}{l}8 \\
8\end{array}$ & $\begin{array}{l}0 \\
0 \\
0\end{array}$ & $\begin{array}{r}-4.493698 \\
-5600799\end{array}$ & $\begin{array}{r}1.590452 \\
-2.975844\end{array}$ & $\begin{array}{l}-1.191527 \\
-0881979\end{array}$ \\
\hline 27 & $\begin{array}{l}0 \\
6\end{array}$ & 0 & $\begin{array}{l}-5.321789 \\
-4.126723\end{array}$ & $\begin{array}{l}-0.101163 \\
2.972015\end{array}$ & $\begin{array}{l}2.464247 / \\
-1.337474\end{array}$ & $\begin{array}{l}26 \\
27\end{array}$ & $\begin{array}{l}8 \\
6\end{array}$ & $\begin{array}{l}0 \\
0\end{array}$ & $\begin{array}{l}-5.6000 / 945 \\
-3.5395\end{array}$ & $\begin{array}{r}-2.9758444 \\
2.642596\end{array}$ & $\begin{array}{l}-0.881919 \\
-1.223237\end{array}$ \\
\hline 28 & 1 & 0 & -3.066309 & 3.230065 & -1.311747 & 28 & 1 & 0 & -2.921561 & 2.624941 & -0.324970 \\
\hline 29 & 1 & 0 & $\begin{array}{l}-4.608403 \\
\end{array}$ & 3.284781 & -0.407484 & & 1 & 0 & -4.116071 & 3.561859 & -1.267312 \\
\hline $\begin{array}{l}30 \\
31\end{array}$ & $\frac{1}{6}$ & $\begin{array}{l}0 \\
0\end{array}$ & $\begin{array}{r}-4.615593 \\
\end{array}$ & $\begin{array}{r}3.456862 \\
-152112\end{array}$ & $\begin{array}{r}-2.177484 \\
355011\end{array}$ & 30 & $\frac{1}{6}$ & 0 & $\begin{array}{l}-2.895128 \\
-65888915\end{array}$ & $\begin{array}{r}2.569470 \\
3.11590\end{array}$ & $\begin{array}{l}-2.102046 \\
\end{array}$ \\
\hline $\begin{array}{l}31 \\
32\end{array}$ & $\begin{array}{l}6 \\
1\end{array}$ & $\begin{array}{l}0 \\
0\end{array}$ & $\begin{array}{r}-4.953406 \\
-4.179168\end{array}$ & $\begin{array}{l}-1.521122 \\
-1.043095\end{array}$ & $\begin{array}{l}3.550119 \\
4.154822\end{array}$ & $\begin{array}{l}31 \\
32\end{array}$ & $\begin{array}{l}6 \\
1\end{array}$ & $\begin{array}{l}0 \\
0\end{array}$ & $\begin{array}{l}-6.5888915 \\
-7.181498\end{array}$ & $\begin{array}{l}-3.1117596 \\
-3.985175\end{array}$ & $\begin{array}{r}-1.910035 \\
-1.640270\end{array}$ \\
\hline 33 & 1 & 0 & -4.596152 & $\begin{array}{l}-1.043095 \\
-2.492917\end{array}$ & 3.201087 & 33 & 1 & $\begin{array}{l}0 \\
0\end{array}$ & $\begin{array}{l}-1.1818988 \\
-6.098086\end{array}$ & $\begin{array}{l}-3.985115 \\
-3.288373\end{array}$ & $\begin{array}{l}-1.640270 \\
-2.86818\end{array}$ \\
\hline 34 & 1 & 0 & -5.848894 & -1.654821 & 4.149550 & 34 & 1 & 0 & -7.223032 & -2.232830 & -1.958021 \\
\hline $\begin{array}{l}35 \\
36\end{array}$ & 1 & 0 & -1.201691 & -2.889030 & 1.359167 & 35 & 1 & 0 & -1.079128 & -3.878712 & -1.054293 \\
\hline $\begin{array}{l}36 \\
37\end{array}$ & $\begin{array}{l}8 \\
8\end{array}$ & $\begin{array}{l}0 \\
0\end{array}$ & 5.427904 & -1.365669 & -0.340539 & 36 & 8 & 0 & 5.326170 & -1.067758 & -0.646107 \\
\hline 38 & $\begin{array}{l}8 \\
6\end{array}$ & $\begin{array}{l}0 \\
0\end{array}$ & $\begin{array}{l}1.901131 \\
6392903\end{array}$ & 1.858965 & $\begin{array}{r}-0.604345 \\
0.59205\end{array}$ & 37 & 8 & 0 & $\begin{array}{l}1.350518 \\
5.55908\end{array}$ & 1.573889 & -1.100015 \\
\hline 39 & 6 & 0 & $\begin{array}{l}6.3992903 \\
7.341237\end{array}$ & $\begin{array}{l}-0.899034 \\
-2.051034\end{array}$ & $\begin{array}{l}0.595205 \\
0.85886\end{array}$ & $\begin{array}{l}38 \\
39 \\
39\end{array}$ & 6 & ${ }_{0}^{0}$ & $\begin{array}{l}5.959498 \\
7149832\end{array}$ & $\begin{array}{r}-0.786929 \\
1736139\end{array}$ & $\begin{array}{l}0.581626 \\
0694972\end{array}$ \\
\hline 40 & 1 & 0 & 5.881428 & -0.601018 & 1.513991 & 40 & $\begin{array}{l}0 \\
1\end{array}$ & 0 & $\begin{array}{l}7.149832 \\
5.241536\end{array}$ & $\begin{array}{l}-1.736138 \\
-0.924852\end{array}$ & $\begin{array}{l}0.694972 \\
1.396604\end{array}$ \\
\hline 41 & 6 & 0 & 8.271213 & 0.005828 & -0.603423 & 41 & 6 & 0 & 7.651502 & 0.865904 & $\begin{array}{l}1.062061 \\
\end{array}$ \\
\hline 42 & 6 & 0 & 8.012517 & -2.488094 & -0.472589 & 42 & 6 & 0 & 8.240413 & -1.208554 & -0.225899 \\
\hline 43 & 1 & 0 & $\begin{array}{l}8.129697 \\
8215947\end{array}$ & $\begin{array}{l}-1.705037 \\
\end{array}$ & $\begin{array}{l}1.508375 \\
1.11205\end{array}$ & 43 & 1 & 0 & $\begin{array}{r}7.529719 \\
\end{array}$ & -1.726705 & $\begin{array}{l}1.719791 \\
0.27890\end{array}$ \\
\hline $\begin{array}{l}44 \\
45\end{array}$ & 6 & $\begin{array}{l}0 \\
0\end{array}$ & $\begin{array}{l}8.219547 \\
9.091315\end{array}$ & $\begin{array}{l}-1.284285 \\
-0.025945\end{array}$ & $\begin{array}{r}-1.412305 \\
01110404\end{array}$ & $\begin{array}{l}44 \\
45\end{array}$ & 6 & $\begin{array}{l}0 \\
0\end{array}$ & $\begin{array}{l}8.789858 \\
7.674400\end{array}$ & $\begin{array}{l}0.091858 \\
0.620332\end{array}$ & 0.378504 \\
\hline $\begin{array}{l}45 \\
46\end{array}$ & $\begin{array}{l}1 \\
1\end{array}$ & $\begin{array}{l}0 \\
0\end{array}$ & $\begin{array}{l}9.0991315 \\
7.381807\end{array}$ & $\begin{array}{r}-0.0235444 \\
-3.234233\end{array}$ & $\begin{array}{r}0.119048 \\
-0.961240\end{array}$ & $\begin{array}{l}45 \\
46\end{array}$ & $\begin{array}{l}1 \\
1\end{array}$ & $\begin{array}{l}0 \\
0\end{array}$ & $\begin{array}{l}7.674400 \\
7.81689\end{array}$ & $\begin{array}{r}0.626339 \\
-1003159\end{array}$ & $\begin{array}{r}2.127437 \\
-1212034\end{array}$ \\
\hline $\begin{array}{l}x_{0} \\
47\end{array}$ & 6 & 0 & 2.260035 & 2.877902 & $\begin{array}{c}-0.901240 \\
0.347693\end{array}$ & $\begin{array}{l}\text { } 10 \\
47\end{array}$ & 6 & 0 & 0.531333 & $\begin{array}{l}-1.008139 \\
1.98482\end{array}$ & $\begin{array}{r}-1.2121234 \\
0.011914\end{array}$ \\
\hline 48 & 6 & 0 & 2.431466 & 4.165110 & -0.447505 & 48 & 6 & 0 & 1.369347 & 2.790427 & 1.006006 \\
\hline 49 & 1 & 0 & 3.217649 & 2.598785 & 0.789299 & 49 & 1 & 0 & 0.131668 & 1.098423 & 0.499938 \\
\hline 50 & 6 & 0 & 2.105772 & 5.330339 & 0.457336 & 50 & 6 & 0 & $\begin{array}{l}0.719215 \\
\end{array}$ & 4.177851 & $\begin{array}{l}0.467540 \\
0.4750\end{array}$ \\
\hline 51 & 6 & 0 & $\begin{array}{l}0.257784 \\
0620792\end{array}$ & $\begin{array}{l}3.867407 \\
524766\end{array}$ & $\begin{array}{l}1.352224 \\
078568\end{array}$ & 51 & 6 & 0 & $\begin{array}{r}-0.338865 \\
\end{array}$ & $\begin{array}{r}3.985627 \\
4876095\end{array}$ & $\begin{array}{l}-1.021203 \\
\end{array}$ \\
\hline $\begin{array}{l}52 \\
53\end{array}$ & 6 & $\begin{array}{l}0 \\
0\end{array}$ & $\begin{array}{c}0.629792 \\
-0.003348\end{array}$ & $\begin{array}{l}5.247665 \\
4017537\end{array}$ & $\begin{array}{l}0.785680 \\
2.399897\end{array}$ & $\begin{array}{l}52 \\
53\end{array}$ & ${ }^{6}$ & $\begin{array}{l}0 \\
0\end{array}$ & $\begin{array}{r}0.460422 \\
-1.347014\end{array}$ & $\begin{array}{l}4.876095 \\
4.395544\end{array}$ & $\begin{array}{c}-0.071762 \\
-107984\end{array}$ \\
\hline 54 & 8 & 0 & 7.031726 & 0.240789 & 0.081179 & 54 & 8 & 0 & $\begin{array}{r}-1.0471714 \\
6.369550\end{array}$ & $\begin{array}{l}4.3995844 \\
0.560823\end{array}$ & $\begin{array}{c}-1.079848 \\
0.515265\end{array}$ \\
\hline 55 & 8 & 0 & 1.360833 & 2.950563 & 1.396184 & 55 & 8 & 0 & -0.556989 & 2.677402 & -0.464103 \\
\hline 56 & 1 & 0 & 2.693561 & 5.257126 & 1.380915 & 56 & 1 & 0 & 2.076450 & 4.770818 & 1.314691 \\
\hline $\begin{array}{l}57 \\
58\end{array}$ & 1 & 0 & $\begin{array}{l}1.732978 \\
0.028027\end{array}$ & $\begin{array}{l}4.164171 \\
3\end{array}$ & $\begin{array}{r}-1.291858 \\
064000\end{array}$ & 57 & 1 & 0 & $\begin{array}{l}2.294706 \\
\end{array}$ & 2.235343 & 1.206219 \\
\hline $\begin{array}{l}58 \\
59\end{array}$ & 6 & $\begin{array}{l}0 \\
0\end{array}$ & $\begin{array}{l}-0.928297 \\
-1.779903\end{array}$ & $\begin{array}{l}3.242384 \\
3.920494\end{array}$ & $\begin{array}{l}0.642000 \\
0.728649\end{array}$ & $\begin{array}{l}58 \\
59\end{array}$ & ${ }_{1}^{6}$ & $\begin{array}{l}0 \\
0\end{array}$ & $\begin{array}{l}0.223080 \\
1.289592\end{array}$ & $\begin{array}{l}3.892850 \\
3.675705\end{array}$ & $\begin{array}{r}-2.438671 \\
-2427767\end{array}$ \\
\hline $\begin{array}{l}59 \\
60\end{array}$ & 1 & 0 & $\begin{array}{l}-1.1 / 79003 \\
-1.186961\end{array}$ & $\begin{array}{l}3.920494 \\
2.300087\end{array}$ & $\begin{array}{l}0.78649 \\
1.134026\end{array}$ & $\begin{array}{l}59 \\
60\end{array}$ & $\begin{array}{l}1 \\
1\end{array}$ & $\begin{array}{l}0 \\
0\end{array}$ & $\begin{array}{l}1.285992 \\
0.079317\end{array}$ & $\begin{array}{l}3.657908 \\
\end{array}$ & $\begin{array}{l}-2.42 / 76 / 1 \\
-2.920101\end{array}$ \\
\hline 61 & 8 & 0 & $\begin{array}{l}-1.180901 \\
-0.692002\end{array}$ & 3.046450 & $\begin{array}{r}1.1 .7402020 \\
-0.736038\end{array}$ & $\begin{array}{l}60 \\
61\end{array}$ & 8 & 0 & -0.489476 & $\begin{array}{l}4.8008090 \\
2.941147\end{array}$ & $\begin{array}{l}-2.920101 \\
-3.199711\end{array}$ \\
\hline 62 & 1 & 0 & -0.000628 & 2.377916 & -0.830901 & 62 & 1 & 0 & -0.258898 & 2.076637 & -2.845036 \\
\hline $\begin{array}{l}62 \\
63\end{array}$ & 1 & 0 & $\begin{array}{c}-0.0008028 \\
0.08137\end{array}$ & 5.411521 & $\begin{array}{l}-0.835910 \\
-0.152780\end{array}$ & $\begin{array}{l}62 \\
63 \\
\end{array}$ & $\begin{array}{l}1 \\
1\end{array}$ & 0 & $\begin{array}{l}-0.238888 \\
0.788623\end{array}$ & $\begin{array}{l}2.0757271 \\
\end{array}$ & $\begin{array}{r}-2.843030 \\
-0.627033\end{array}$ \\
\hline 64 & 8 & 0 & $\begin{array}{l}0.252881 \\
0.52166\end{array}$ & 6.218185 & 1.739102 & 64 & 8 & 0 & -0.352112 & 5.331658 & 0.988426 \\
\hline $\begin{array}{l}65 \\
66\end{array}$ & $\begin{array}{l}1 \\
8\end{array}$ & $\begin{array}{l}0 \\
0\end{array}$ & $\begin{array}{l}0.521668 \\
2.3229298\end{array}$ & $\begin{array}{l}7.076560 \\
6560031\end{array}$ & $\begin{array}{c}1.396480 \\
-0.175394\end{array}$ & 65 & ${ }_{8}^{1}$ & $\begin{array}{l}0 \\
0\end{array}$ & $\begin{array}{r}-0.368230 \\
2705058\end{array}$ & $\begin{array}{l}4.641647 \\
\end{array}$ & $\begin{array}{l}1.662435 \\
053720\end{array}$ \\
\hline $\begin{array}{l}60 \\
67\end{array}$ & 1 & $\begin{array}{l}0 \\
0\end{array}$ & $\begin{array}{l}2.3229297 \\
3.212771\end{array}$ & $\begin{array}{l}6.565031 \\
6.551251\end{array}$ & $\begin{array}{l}-0.1753944 \\
-0.556769\end{array}$ & $\begin{array}{l}66 \\
67\end{array}$ & $\begin{array}{l}8 \\
1\end{array}$ & $\begin{array}{l}0 \\
0\end{array}$ & $\begin{array}{l}2.705058 \\
3.056492\end{array}$ & $\begin{array}{l}4.138655 \\
3.247265\end{array}$ & $\begin{array}{l}-0.5372266 \\
-0.649402\end{array}$ \\
\hline 68 & 8 & 0 & 3.760539 & 4.302004 & -0.897529 & 68 & 8 & 0 & $\begin{array}{l}3.0305942 \\
0.627923\end{array}$ & $\begin{array}{l}3.247265 \\
2.984002\end{array}$ & $\begin{array}{c}-0.649402 \\
2.194068\end{array}$ \\
\hline 69 & 1 & 0 & 4.025253 & 3.488078 & -1.340621 & 69 & 1 & 0 & 0.229485 & 2.147128 & 2.471969 \\
\hline 70 & 1 & 0 & $\begin{array}{l}8.427238 \\
7357520\end{array}$ & 0.855520 & -1.262857 & 70 & 1 & 0 & 7.767540 & 1.939769 & 0.949540 \\
\hline $\begin{array}{l}71 \\
72 \\
72\end{array}$ & $\begin{array}{l}1 \\
8\end{array}$ & $\begin{array}{l}0 \\
0 \\
0\end{array}$ & $\begin{array}{l}7.357522 \\
9371982\end{array}$ & $\begin{array}{l}-1.225812 \\
-1.407725\end{array}$ & $\begin{array}{l}-2.079269 \\
-2.199193\end{array}$ & $\begin{array}{l}71 \\
72\end{array}$ & $\begin{array}{l}1 \\
8\end{array}$ & $\begin{array}{l}0 \\
0\end{array}$ & $\begin{array}{l}9.246392 \\
9732207\end{array}$ & 0.692487 & -0.413328 \\
\hline 73 & $\begin{array}{l}8 \\
1\end{array}$ & 0 & $\begin{array}{l}9.371982 \\
9.977527\end{array}$ & $\begin{array}{l}-1.400 / 23 \\
-2.000372\end{array}$ & $\begin{array}{l}-2.199193 \\
-1.739291\end{array}$ & $\begin{array}{l}12 \\
73\end{array}$ & $\begin{array}{l}8 \\
1\end{array}$ & $\begin{array}{l}0 \\
0\end{array}$ & $\begin{array}{r}9.73272707 \\
10.261621\end{array}$ & $\begin{array}{l}-0.1866777 \\
-0.931700\end{array}$ & $\begin{array}{l}1.384681 \\
1.075158\end{array}$ \\
\hline 74 & 8 & 0 & 9.289286 & -3.035492 & -0.193732 & 74 & $\begin{array}{l}1 \\
8\end{array}$ & 0 & 9.301462 & $\begin{array}{l}-0.9312650 \\
-2.12636\end{array}$ & $\begin{array}{r}1.333552 \\
-0.335\end{array}$ \\
\hline 75 & 1 & 0 & 9.178259 & -3.808634 & 0.366064 & 75 & 1 & 0 & 8.915108 & -3.010033 & -0.341049 \\
\hline 76 & 8 & 0 & 6.692044 & -3.111147 & 1.483002 & 76 & 8 & 0 & 6.791401 & -3.059889 & 0.403824 \\
\hline 77 & 1 & 0 & 5.837571 & -3.272187 & 1.055048 & 77 & 1 & 0 & 6.054374 & -3.068617 & -0.223446 \\
\hline 78 & 6 & 0 & -6.850162 & 0.295435 & -0.767835 & 78 & 6 & 0 & -6.726759 & 0.359306 & 0.291850 \\
\hline $\begin{array}{l}79 \\
80\end{array}$ & 6 & 0 & $\begin{array}{r}-7.270644 \\
7.932000\end{array}$ & $\begin{array}{r}-1.172678 \\
1.273684\end{array}$ & $\begin{array}{l}-0.656106 \\
\end{array}$ & 79 & 6 & 0 & $\begin{array}{r}-6.231029 \\
\end{array}$ & -0.334620 & 1.576920 \\
\hline $\begin{array}{l}80 \\
81\end{array}$ & 8 & $\begin{array}{l}0 \\
0 \\
0\end{array}$ & $\begin{array}{l}-7.932009 \\
-7.986297\end{array}$ & $\begin{array}{l}-1.273684 \\
-1.425183\end{array}$ & $\begin{array}{r}0.212898 \\
-1.859203\end{array}$ & $\begin{array}{l}80 \\
81\end{array}$ & $\begin{array}{l}1 \\
8\end{array}$ & $\begin{array}{l}0 \\
0\end{array}$ & $\begin{array}{l}-6.7864977 \\
\end{array}$ & $\begin{array}{l}0.128788 \\
169746\end{array}$ & $\begin{array}{l}2.397698 \\
1590009\end{array}$ \\
\hline $\begin{array}{l}11 \\
82\end{array}$ & $\begin{array}{l}0 \\
1\end{array}$ & 0 & $\begin{array}{l}-7.986297 \\
-8.105819\end{array}$ & $\begin{array}{l}-1.425183 \\
-2.373913\end{array}$ & $\begin{array}{l}-1.859203 \\
-1.946453\end{array}$ & $\begin{array}{l}81 \\
82\end{array}$ & $\begin{array}{l}8 \\
1\end{array}$ & $\begin{array}{l}0 \\
0\end{array}$ & $\begin{array}{l}-6.60001617 \\
-6.331623\end{array}$ & $\begin{array}{l}-1.694766 \\
-2.122185\end{array}$ & $\begin{array}{l}1.590005 \\
0.760598\end{array}$ \\
\hline 83 & 6 & 0 & $\begin{array}{l}-8.100819 \\
-6.111987\end{array}$ & $\begin{array}{l}-2.123209 \\
-\end{array}$ & $\begin{array}{l}-1.9440433 \\
-0.48090\end{array}$ & 83 & 6 & 0 & $\begin{array}{l}-0.031039 \\
-4.748469\end{array}$ & -0.118069 & 1.860641 \\
\hline 84 & 6 & 0 & -5.041566 & -2.094856 & -1.383682 & 84 & 6 & 0 & -3.888654 & -1.207418 & 1.987696 \\
\hline 85 & 6 & 0 & -6.075277 & -3.004215 & 0.584322 & 85 & 6 & 0 & -4.241565 & 1.166542 & 2.000701 \\
\hline 86 & 6 & 0 & -3.933372 & -2.886731 & -1.169006 & 86 & 6 & 0 & -2.541107 & -1.001872 & 2.219400 \\
\hline 87 & 1 & 0 & -5.073035 & -1.413141 & $\begin{array}{l}-2.222999 \\
\end{array}$ & 87 & 1 & 0 & -4.285391 & -2.207465 & 1.893266 \\
\hline $\begin{array}{l}88 \\
89\end{array}$ & 6 & $\begin{array}{l}0 \\
0\end{array}$ & $\begin{array}{l}-4.974018 \\
-6.890988\end{array}$ & $\begin{array}{l}-3.833735 \\
-3028025\end{array}$ & $\begin{array}{l}0.786231 \\
1286410\end{array}$ & $\begin{array}{l}88 \\
89\end{array}$ & ${ }^{6}$ & $\begin{array}{l}0 \\
0\end{array}$ & $\begin{array}{l}-2.8900036 \\
-4890702\end{array}$ & $\begin{array}{l}1.377410 \\
2.023936\end{array}$ & $\begin{array}{l}2.256934 \\
1.912414\end{array}$ \\
\hline 90 & 6 & 0 & $\begin{array}{l}-0.899080 \\
-3.896103\end{array}$ & $\begin{array}{r}-3.028029 \\
-3.763026\end{array}$ & $\begin{array}{r}1.286410 \\
-0.070678\end{array}$ & 90 & 6 & $\begin{array}{l}0 \\
0\end{array}$ & $\begin{array}{l}-4.8970828 \\
-2.044640\end{array}$ & $\begin{array}{l}2.023936 \\
0.294650\end{array}$ & $\begin{array}{l}1.912415 \\
2.371182\end{array}$ \\
\hline 91 & 1 & 0 & -4.933948 & -4.532812 & 1.610687 & 91 & 1 & 0 & -2.487920 & 2.375823 & 2.372627 \\
\hline 92 & 8 & 0 & -2.801144 & -4.529529 & 0.145920 & 92 & 8 & 0 & -0.713025 & 0.465081 & 2.645732 \\
\hline 93 & 1 & 0 & $\begin{array}{l}-2.166749 \\
\end{array}$ & $\begin{array}{r}-4.360590 \\
\end{array}$ & -0.561401 & 93 & 1 & 0 & -0.322592 & -0.410724 & 2.760879 \\
\hline $\begin{array}{l}94 \\
95\end{array}$ & $\begin{array}{l}8 \\
6\end{array}$ & $\begin{array}{l}0 \\
0\end{array}$ & $\begin{array}{l}-2.8075577 \\
-2.77545\end{array}$ & $\begin{array}{l}-2.904579 \\
-2.047115\end{array}$ & $\begin{array}{r}-1.934141 \\
-3.058014\end{array}$ & $\begin{array}{l}94 \\
95\end{array}$ & $\begin{array}{l}8 \\
6\end{array}$ & $\begin{array}{l}0 \\
0\end{array}$ & $\begin{array}{l}-1.5916424 \\
-2013998\end{array}$ & $\begin{array}{l}-1.974489 \\
-3.318799\end{array}$ & $\begin{array}{r}2.323275 \\
2.197811\end{array}$ \\
\hline $\begin{array}{l}93 \\
96\end{array}$ & $\begin{array}{l}6 \\
1\end{array}$ & $\begin{array}{l}0 \\
0\end{array}$ & $\begin{array}{l}-2.27 / 744 / 1 \\
-3.559873\end{array}$ & $\begin{array}{l}-2.04 / 113 \\
-2.312279\end{array}$ & $\begin{array}{l}-3.050814 \\
-3.76881\end{array}$ & $\begin{array}{l}95 \\
96\end{array}$ & $\begin{array}{l}6 \\
1\end{array}$ & $\begin{array}{l}0 \\
0\end{array}$ & $\begin{array}{l}-2.0130988 \\
-1.118712\end{array}$ & $\begin{array}{r}-3.318191 \\
-3.929584\end{array}$ & $\begin{array}{l}2.198111 \\
2.264127\end{array}$ \\
\hline 97 & 1 & 0 & -1.801889 & -2.185685 & -3.520116 & $\begin{array}{l}90 \\
97\end{array}$ & 1 & 0 & -2.498204 & $\begin{array}{l}-3.479067 \\
-\end{array}$ & 1.232432 \\
\hline 98 & 1 & 0 & -2.895108 & -1.004871 & -2.754129 & 98 & 1 & 0 & -2.705234 & -3.582823 & 3.000255 \\
\hline 99 & 6 & 0 & -8.049808 & $\begin{array}{l}1.219594 \\
\end{array}$ & -0.837883 & 99 & 6 & 0 & -8.243586 & 0.449297 & 0.229095 \\
\hline
\end{tabular}




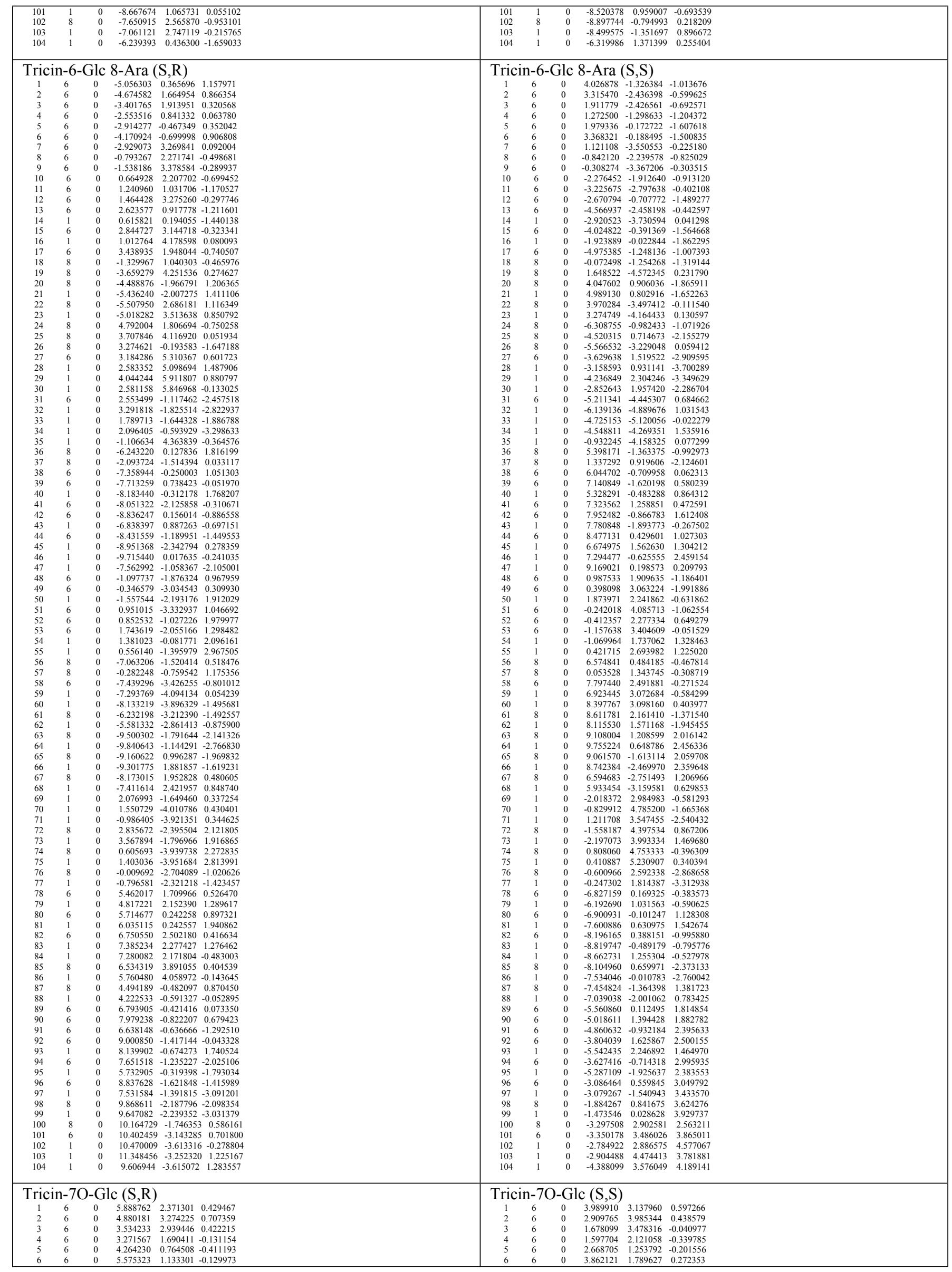




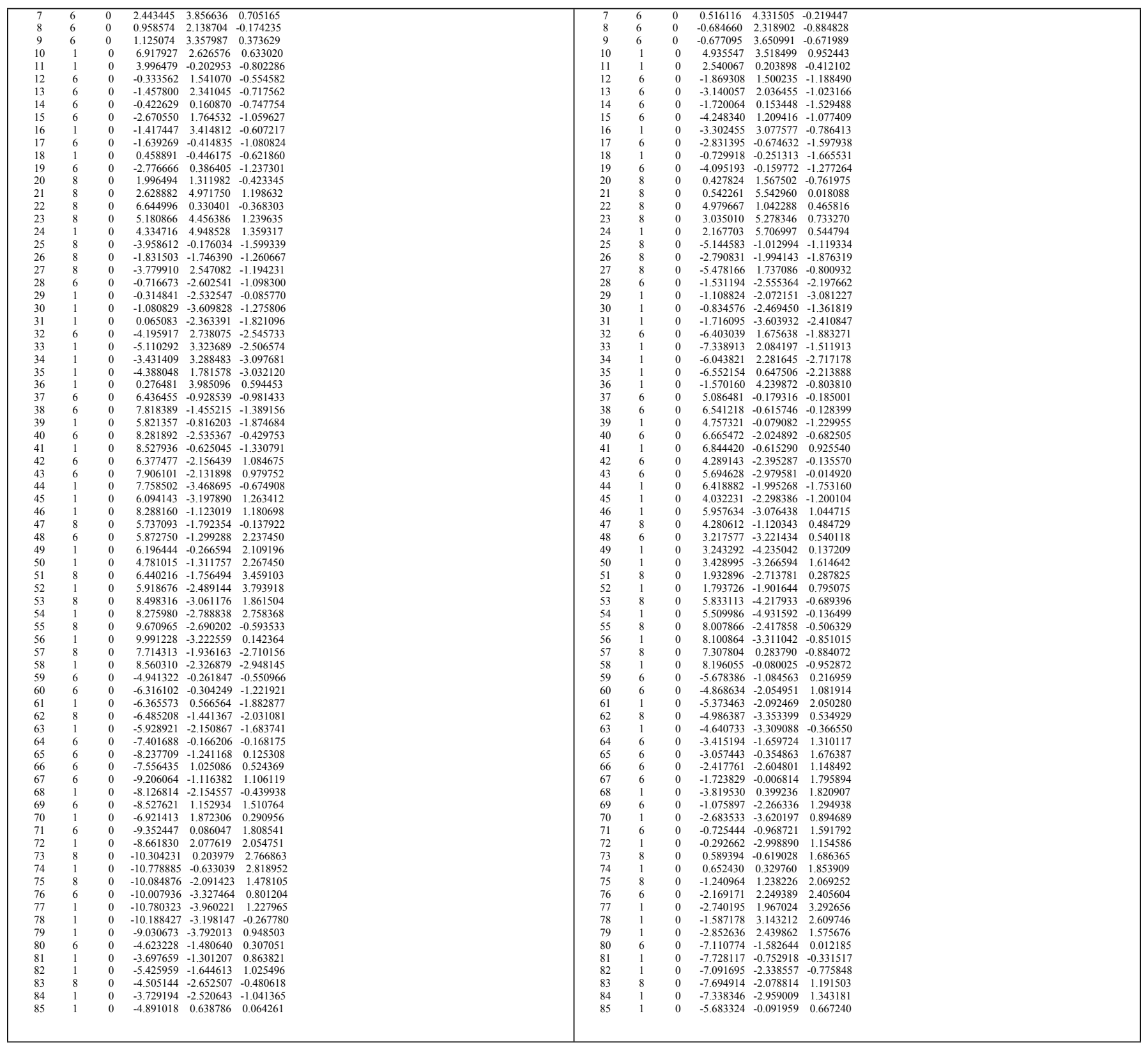

Flavanol-lignin dimers

\begin{tabular}{|c|c|c|c|c|c|c|c|c|c|c|c|}
\hline \multicolumn{6}{|c|}{ 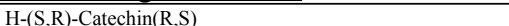 } & \multicolumn{6}{|c|}{ H-(S,S)-Catechin(R,S) } \\
\hline 1 & 6 & 0 & -0.633328 & 1.929098 & -0.530539 & 1 & 6 & 0 & -0.507370 & 0.497654 & 1.903375 \\
\hline 2 & 6 & 0 & 0.368420 & 2.034267 & 0.436697 & 2 & 6 & 0 & 0.513093 & -0.442415 & 2.073257 \\
\hline 3 & 6 & 0 & 1.687328 & 1.718063 & 0.140982 & 3 & 6 & 0 & 1.802863 & -0.169116 & 1.643423 \\
\hline 4 & 6 & 0 & 2.016093 & 1.262090 & -1.146359 & 4 & 6 & 0 & 2.079630 & 1.064604 & 1.040424 \\
\hline 5 & 6 & 0 & 1.018554 & 1.154662 & -2.095650 & 5 & 6 & 0 & 1.075665 & 1.995982 & 0.886632 \\
\hline 6 & 6 & 0 & -0.298585 & 1.482500 & -1.798538 & 6 & 6 & 0 & -0.219232 & 1.714770 & 1.307766 \\
\hline 7 & 1 & 0 & 0.127007 & 2.390608 & 1.426640 & 7 & 1 & 0 & 0.300102 & -1.392001 & 2.541288 \\
\hline 8 & 1 & 0 & 1.299194 & 0.807960 & -3.081380 & 8 & 1 & 0 & 1.317068 & 2.945916 & 0.425764 \\
\hline 9 & 1 & 0 & -1.061780 & 1.399563 & -2.559139 & 9 & 1 & 0 & -0.998792 & 2.451534 & 1.180986 \\
\hline 10 & 6 & 0 & -2.044131 & 2.403539 & -0.240012 & 10 & 6 & 0 & -1.902103 & 0.207663 & 2.423216 \\
\hline 11 & 1 & 0 & -2.119769 & 3.470365 & -0.455641 & 11 & 1 & 0 & -1.939283 & 0.377696 & 3.500087 \\
\hline 12 & 6 & 0 & -2.541078 & 2.173393 & 1.187312 & 12 & 6 & 0 & -2.411025 & -1.208049 & 2.141841 \\
\hline 13 & 6 & 0 & -2.540368 & 0.681761 & 1.489528 & 13 & 6 & 0 & -2.438430 & -1.440167 & 0.638148 \\
\hline 14 & 1 & 0 & -1.901051 & 2.699979 & 1.892572 & 14 & 1 & 0 & -1.760794 & -1.938600 & 2.619139 \\
\hline 15 & 1 & 0 & -1.512909 & 0.348815 & 1.657642 & 15 & 1 & 0 & -1.416660 & -1.595135 & 0.283300 \\
\hline 16 & 1 & 0 & -3.098389 & 0.501548 & 2.408432 & 16 & 1 & 0 & -3.000934 & -2.349170 & 0.424724 \\
\hline 17 & 8 & 0 & -2.982993 & 1.811926 & -1.144071 & 17 & 8 & 0 & -2.852174 & 1.136794 & 1.897648 \\
\hline 18 & 8 & 0 & -3.820157 & 2.750265 & 1.340675 & 18 & 8 & 0 & -3.676385 & -1.383468 & 2.740616 \\
\hline 19 & 1 & 0 & -4.381564 & 2.448506 & 0.619510 & 19 & 1 & 0 & -4.247356 & -0.656983 & 2.471250 \\
\hline 20 & 8 & 0 & 3.298429 & 0.994327 & -1.526085 & 20 & 8 & 0 & 3.362693 & 1.317516 & 0.636278 \\
\hline 21 & 8 & 0 & 2.709028 & 1.828588 & 1.021395 & 21 & 8 & 0 & 2.849322 & -1.024026 & 1.749515 \\
\hline 22 & 6 & 0 & 2.399369 & 2.177592 & 2.352454 & 22 & 6 & 0 & 2.578671 & -2.336779 & 2.197740 \\
\hline 23 & 1 & 0 & 3.337417 & 2.147326 & 2.899076 & 23 & 1 & 0 & 3.516719 & -2.880146 & 2.128202 \\
\hline 24 & 1 & 0 & 1.697496 & 1.461591 & 2.787994 & 24 & 1 & 0 & 1.830779 & -2.814617 & 1.560656 \\
\hline 25 & 1 & 0 & 1.980907 & 3.185235 & 2.408653 & 25 & 1 & 0 & 2.238390 & -2.333078 & 3.235444 \\
\hline 26 & 6 & 0 & -3.152864 & -0.074117 & 0.342274 & 26 & 6 & 0 & -3.056442 & -0.257220 & -0.055903 \\
\hline 27 & 6 & 0 & -3.586867 & -1.391740 & 0.475893 & 27 & 6 & 0 & -3.508844 & -0.325288 & -1.372793 \\
\hline 28 & 6 & 0 & -3.333017 & 0.516997 & -0.905171 & 28 & 6 & 0 & -3.228650 & 0.959274 & 0.598176 \\
\hline
\end{tabular}




\begin{tabular}{|c|}
\hline \\
\hline 29 \\
\hline 30 \\
\hline 31 \\
\hline 32 \\
\hline 33 \\
\hline 34 \\
\hline 35 \\
\hline 36 \\
\hline 37 \\
\hline 38 \\
\hline 39 \\
\hline 40 \\
\hline 41 \\
\hline 42 \\
\hline 43 \\
\hline 44 \\
\hline 45 \\
\hline 46 \\
\hline 47 \\
\hline 48 \\
\hline 49 \\
\hline 50 \\
\hline 51 \\
\hline 52 \\
\hline 53 \\
\hline 54 \\
\hline 55 \\
\hline 56 \\
\hline 57 \\
\hline 58 \\
\hline 59 \\
\hline 60 \\
\hline $\mathrm{H}-(\mathrm{S}, \mathrm{R}$ \\
\hline 1 \\
\hline 2 \\
\hline 3 \\
\hline 4 \\
\hline 5 \\
\hline 6 \\
\hline 7 \\
\hline 8 \\
\hline 9 \\
\hline 10 \\
\hline 11 \\
\hline 12 \\
\hline 13 \\
\hline 14 \\
\hline 15 \\
\hline 16 \\
\hline 17 \\
\hline 18 \\
\hline 19 \\
\hline 20 \\
\hline 21 \\
\hline 22 \\
\hline 23 \\
\hline 24 \\
\hline 25 \\
\hline 26 \\
\hline 27 \\
\hline 28 \\
\hline 29 \\
\hline 30 \\
\hline 31 \\
\hline 32 \\
\hline 33 \\
\hline 34 \\
\hline 35 \\
\hline 36 \\
\hline 37 \\
\hline 38 \\
\hline 39 \\
\hline 40 \\
\hline 41 \\
\hline 42 \\
\hline 43 \\
\hline 44 \\
\hline 45 \\
\hline 46 \\
\hline 47 \\
\hline 48 \\
\hline 49 \\
\hline 50 \\
\hline 51 \\
\hline 52 \\
\hline 53 \\
\hline 54 \\
\hline 55 \\
\hline 56 \\
\hline 57 \\
\hline 58 \\
\hline 59 \\
\hline 60 \\
\hline $\mathrm{H}-(\mathrm{S}, \mathrm{R}$ \\
\hline 1 \\
\hline 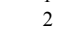 \\
\hline 3 \\
\hline
\end{tabular}




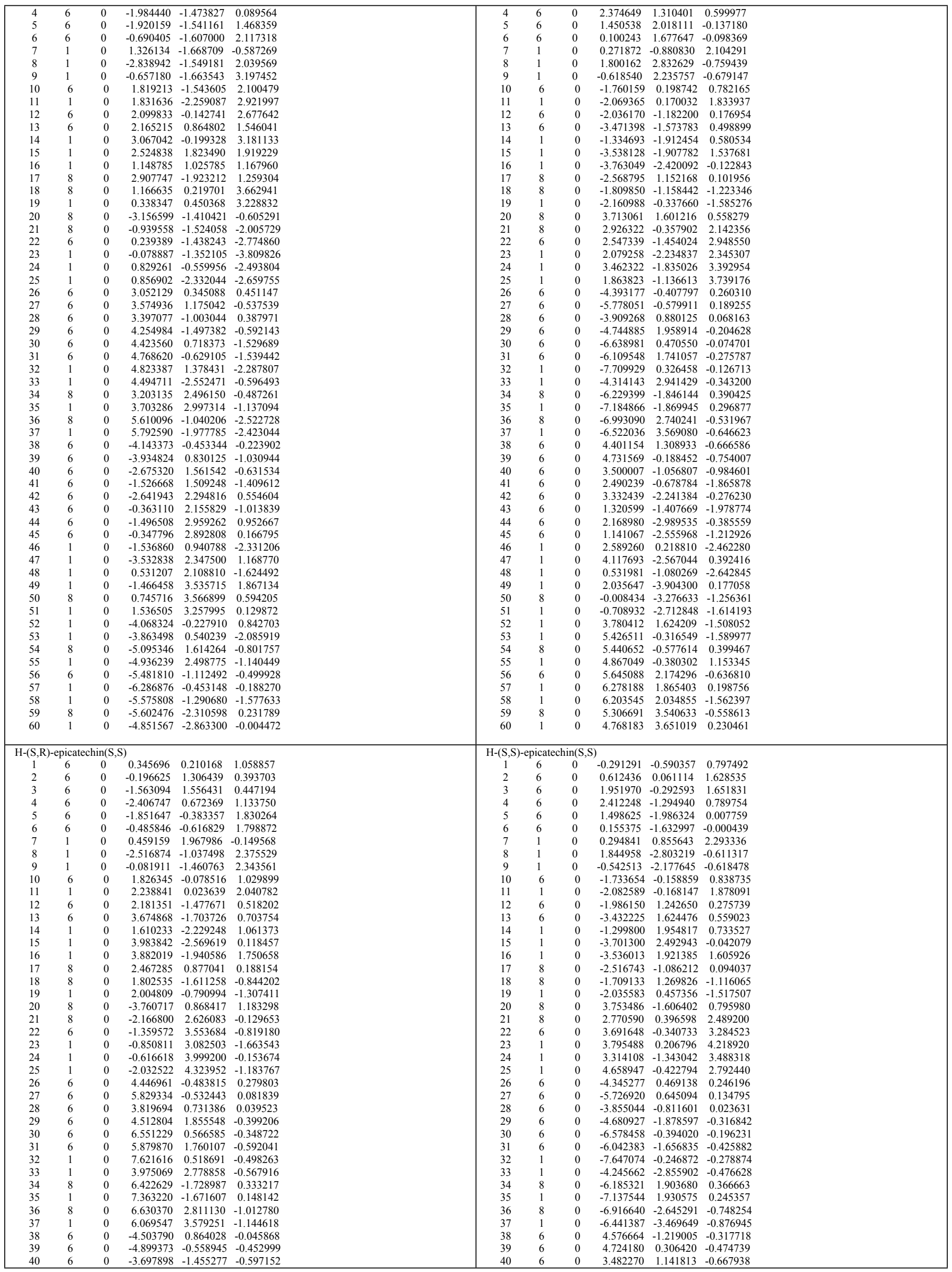




\begin{tabular}{|c|c|}
\hline 41 & 6 \\
\hline 42 & 6 \\
\hline 43 & 6 \\
\hline 44 & 6 \\
\hline 45 & 6 \\
\hline 46 & 1 \\
\hline 47 & 1 \\
\hline 48 & 1 \\
\hline 49 & 1 \\
\hline 50 & 8 \\
\hline 51 & 1 \\
\hline 52 & 1 \\
\hline 53 & 1 \\
\hline 54 & 8 \\
\hline 55 & 1 \\
\hline 56 & 6 \\
\hline 57 & 1 \\
\hline 58 & 1 \\
\hline 59 & 8 \\
\hline 60 & 1 \\
\hline H-(S,R & piga \\
\hline 1 & 6 \\
\hline 2 & 6 \\
\hline 3 & 6 \\
\hline 4 & 6 \\
\hline 5 & 6 \\
\hline 6 & 6 \\
\hline 7 & 1 \\
\hline 8 & 8 \\
\hline 9 & 1 \\
\hline 10 & 6 \\
\hline 11 & 1 \\
\hline 12 & 6 \\
\hline 13 & 6 \\
\hline 14 & 1 \\
\hline 15 & 1 \\
\hline 16 & 1 \\
\hline 17 & 8 \\
\hline 18 & 8 \\
\hline 19 & 1 \\
\hline 20 & 8 \\
\hline 21 & 8 \\
\hline 22 & 1 \\
\hline 23 & 1 \\
\hline 24 & 6 \\
\hline 25 & 6 \\
\hline 26 & 6 \\
\hline 27 & 6 \\
\hline 28 & 6 \\
\hline 29 & 6 \\
\hline 30 & 1 \\
\hline 31 & 1 \\
\hline 32 & 8 \\
\hline 33 & 1 \\
\hline 34 & 8 \\
\hline 35 & 1 \\
\hline 36 & 6 \\
\hline 37 & 6 \\
\hline 38 & 6 \\
\hline 39 & 6 \\
\hline 40 & 6 \\
\hline 41 & 6 \\
\hline 42 & 6 \\
\hline 43 & 6 \\
\hline 44 & 1 \\
\hline 45 & 1 \\
\hline 46 & 1 \\
\hline 47 & 1 \\
\hline 48 & 8 \\
\hline 49 & 1 \\
\hline 50 & 1 \\
\hline 51 & 1 \\
\hline 52 & 8 \\
\hline 53 & 1 \\
\hline 54 & 6 \\
\hline 55 & 1 \\
\hline 56 & 1 \\
\hline 57 & 8 \\
\hline 58 & 1 \\
\hline $\mathrm{H}-(\mathrm{S}, \mathrm{R}$ & $\overline{\text { piga }}$ \\
\hline 1 & 6 \\
\hline 2 & 6 \\
\hline 3 & 6 \\
\hline 4 & 6 \\
\hline 5 & 6 \\
\hline 6 & 6 \\
\hline 7 & 1 \\
\hline 8 & 8 \\
\hline 9 & 1 \\
\hline 10 & 6 \\
\hline 11 & 1 \\
\hline 12 & 6 \\
\hline 13 & 6 \\
\hline 14 & 1 \\
\hline 15 & 1 \\
\hline 16 & 1 \\
\hline 17 & 8 \\
\hline
\end{tabular}




\begin{tabular}{|c|}
\hline 18 \\
\hline 19 \\
\hline 20 \\
\hline 21 \\
\hline 22 \\
\hline 23 \\
\hline 24 \\
\hline 25 \\
\hline 26 \\
\hline 27 \\
\hline 28 \\
\hline 29 \\
\hline 30 \\
\hline 31 \\
\hline 32 \\
\hline 33 \\
\hline 34 \\
\hline 35 \\
\hline 36 \\
\hline 37 \\
\hline 38 \\
\hline 39 \\
\hline 40 \\
\hline 41 \\
\hline 42 \\
\hline 43 \\
\hline 44 \\
\hline 45 \\
\hline 46 \\
\hline 47 \\
\hline 48 \\
\hline 49 \\
\hline 50 \\
\hline 51 \\
\hline 52 \\
\hline 53 \\
\hline 54 \\
\hline 55 \\
\hline 56 \\
\hline 57 \\
\hline 58 \\
\hline 59 \\
\hline 60 \\
\hline 61 \\
\hline 62 \\
\hline 63 \\
\hline 64 \\
\hline 65 \\
\hline 66 \\
\hline 67 \\
\hline 68 \\
\hline 69 \\
\hline 70 \\
\hline 71 \\
\hline 72 \\
\hline 73 \\
\hline $\mathrm{C}-(\mathrm{S}, \mathrm{R}$ \\
\hline 1 \\
\hline 2 \\
\hline 3 \\
\hline 4 \\
\hline 5 \\
\hline 6 \\
\hline 7 \\
\hline 8 \\
\hline 9 \\
\hline 10 \\
\hline 11 \\
\hline 12 \\
\hline 13 \\
\hline 14 \\
\hline 15 \\
\hline 16 \\
\hline 17 \\
\hline 18 \\
\hline 19 \\
\hline 20 \\
\hline 21 \\
\hline 22 \\
\hline 23 \\
\hline 24 \\
\hline 25 \\
\hline 26 \\
\hline 27 \\
\hline 28 \\
\hline 29 \\
\hline 30 \\
\hline 31 \\
\hline 32 \\
\hline 33 \\
\hline 34 \\
\hline 35 \\
\hline 36 \\
\hline 37 \\
\hline 38 \\
\hline 39 \\
\hline 40 \\
\hline 41 \\
\hline
\end{tabular}




\begin{tabular}{|c|c|c|}
\hline 42 & 6 & 0 \\
\hline 43 & 6 & 0 \\
\hline 44 & 6 & 0 \\
\hline 45 & 6 & 0 \\
\hline 46 & 1 & 0 \\
\hline 47 & 1 & 0 \\
\hline 48 & 1 & 0 \\
\hline 49 & 8 & 0 \\
\hline 50 & 8 & 0 \\
\hline 51 & 1 & 0 \\
\hline 52 & 1 & 0 \\
\hline 53 & 1 & 0 \\
\hline 54 & 8 & 0 \\
\hline 55 & 1 & 0 \\
\hline 56 & 6 & 0 \\
\hline 57 & 1 & 0 \\
\hline 58 & 1 & 0 \\
\hline 59 & 8 & 0 \\
\hline 60 & 1 & 0 \\
\hline 61 & 1 & 0 \\
\hline C-(S,R & atec & $\mathrm{S}, \mathrm{R}$ \\
\hline 1 & 6 & 0 \\
\hline 2 & 6 & 0 \\
\hline 3 & 6 & 0 \\
\hline 4 & 6 & 0 \\
\hline 5 & 6 & 0 \\
\hline 6 & 6 & 0 \\
\hline 7 & 1 & 0 \\
\hline 8 & 1 & 0 \\
\hline 9 & 1 & 0 \\
\hline 10 & 6 & 0 \\
\hline 11 & 1 & 0 \\
\hline 12 & 6 & 0 \\
\hline 13 & 6 & 0 \\
\hline 14 & 1 & 0 \\
\hline 15 & 1 & 0 \\
\hline 16 & 1 & 0 \\
\hline 17 & 8 & 0 \\
\hline 18 & 8 & 0 \\
\hline 19 & 1 & 0 \\
\hline 20 & 8 & 0 \\
\hline 21 & 8 & 0 \\
\hline 22 & 6 & 0 \\
\hline 23 & 1 & 0 \\
\hline 24 & 1 & 0 \\
\hline 25 & 1 & 0 \\
\hline 26 & 6 & 0 \\
\hline 27 & 6 & 0 \\
\hline 28 & 6 & 0 \\
\hline 29 & 6 & 0 \\
\hline 30 & 6 & 0 \\
\hline 31 & 6 & 0 \\
\hline 32 & 1 & 0 \\
\hline 33 & 1 & 0 \\
\hline 34 & 8 & 0 \\
\hline 35 & 1 & 0 \\
\hline 36 & 8 & 0 \\
\hline 37 & 1 & 0 \\
\hline 38 & 6 & 0 \\
\hline 39 & 6 & 0 \\
\hline 40 & 6 & 0 \\
\hline 41 & 6 & 0 \\
\hline 42 & 6 & 0 \\
\hline 43 & 6 & 0 \\
\hline 44 & 6 & 0 \\
\hline 45 & 6 & 0 \\
\hline 46 & 1 & 0 \\
\hline 47 & 1 & 0 \\
\hline 48 & 1 & 0 \\
\hline 49 & 8 & 0 \\
\hline 50 & 8 & 0 \\
\hline 51 & 1 & 0 \\
\hline 52 & 1 & 0 \\
\hline 53 & 1 & 0 \\
\hline 54 & 8 & 0 \\
\hline 55 & 1 & 0 \\
\hline 56 & 6 & 0 \\
\hline 57 & 1 & 0 \\
\hline 58 & 1 & 0 \\
\hline 59 & 8 & 0 \\
\hline 60 & 1 & 0 \\
\hline 61 & 1 & 0 \\
\hline C-(S,R & pica & $\operatorname{in}(\mathrm{P}$ \\
\hline 1 & 6 & 0 \\
\hline 2 & 6 & 0 \\
\hline 3 & 6 & 0 \\
\hline 4 & 6 & 0 \\
\hline 5 & 6 & 0 \\
\hline 6 & 6 & 0 \\
\hline 7 & 1 & 0 \\
\hline 8 & 1 & 0 \\
\hline 9 & 1 & 0 \\
\hline 10 & 6 & 0 \\
\hline 11 & 1 & 0 \\
\hline 12 & 6 & 0 \\
\hline 13 & 6 & 0 \\
\hline
\end{tabular}




\begin{tabular}{|c|}
\hline 14 \\
\hline 15 \\
\hline 16 \\
\hline 17 \\
\hline 18 \\
\hline 19 \\
\hline 20 \\
\hline 21 \\
\hline 22 \\
\hline 23 \\
\hline 24 \\
\hline 25 \\
\hline 26 \\
\hline 27 \\
\hline 28 \\
\hline 29 \\
\hline 30 \\
\hline 31 \\
\hline 32 \\
\hline 33 \\
\hline 34 \\
\hline 35 \\
\hline 36 \\
\hline 37 \\
\hline 38 \\
\hline 39 \\
\hline 40 \\
\hline 41 \\
\hline 42 \\
\hline 43 \\
\hline 44 \\
\hline 45 \\
\hline 46 \\
\hline 47 \\
\hline 48 \\
\hline 49 \\
\hline 50 \\
\hline 51 \\
\hline 52 \\
\hline 53 \\
\hline 54 \\
\hline 55 \\
\hline 56 \\
\hline 57 \\
\hline 58 \\
\hline 59 \\
\hline 60 \\
\hline 61 \\
\hline C-(S,R \\
\hline 1 \\
\hline 2 \\
\hline 3 \\
\hline 4 \\
\hline 5 \\
\hline 6 \\
\hline 7 \\
\hline 8 \\
\hline 9 \\
\hline 10 \\
\hline 11 \\
\hline 12 \\
\hline 13 \\
\hline 14 \\
\hline 15 \\
\hline 16 \\
\hline 17 \\
\hline 18 \\
\hline 19 \\
\hline 20 \\
\hline 21 \\
\hline 22 \\
\hline 23 \\
\hline 24 \\
\hline 25 \\
\hline 26 \\
\hline 27 \\
\hline 28 \\
\hline 29 \\
\hline 30 \\
\hline 31 \\
\hline 32 \\
\hline 33 \\
\hline 34 \\
\hline 35 \\
\hline 36 \\
\hline 37 \\
\hline 38 \\
\hline 39 \\
\hline 40 \\
\hline 41 \\
\hline 42 \\
\hline 43 \\
\hline 44 \\
\hline 45 \\
\hline 46 \\
\hline 47 \\
\hline 48 \\
\hline 49 \\
\hline
\end{tabular}




\begin{tabular}{|c|c|c|}
\hline 50 & 8 & 0 \\
\hline 51 & 1 & 0 \\
\hline 52 & 1 & 0 \\
\hline 53 & 1 & 0 \\
\hline 54 & 8 & 0 \\
\hline 55 & 1 & 0 \\
\hline 56 & 6 & 0 \\
\hline 57 & 1 & 0 \\
\hline 58 & 1 & 0 \\
\hline 59 & 8 & 0 \\
\hline 60 & 1 & 0 \\
\hline 61 & 1 & 0 \\
\hline C-(S,R & $\overline{\text { piga }}$ & $\overline{\text { atec }}$ \\
\hline 1 & 6 & 0 \\
\hline 2 & 6 & 0 \\
\hline 3 & 6 & 0 \\
\hline 4 & 6 & 0 \\
\hline 5 & 6 & 0 \\
\hline 6 & 6 & 0 \\
\hline 7 & 1 & 0 \\
\hline 8 & 8 & 0 \\
\hline 9 & 1 & 0 \\
\hline 10 & 6 & 0 \\
\hline 11 & 1 & 0 \\
\hline 12 & 6 & 0 \\
\hline 13 & 6 & 0 \\
\hline 14 & 1 & 0 \\
\hline 15 & 1 & 0 \\
\hline 16 & 1 & 0 \\
\hline 17 & 8 & 0 \\
\hline 18 & 8 & 0 \\
\hline 19 & 1 & 0 \\
\hline 20 & 8 & 0 \\
\hline 21 & 8 & 0 \\
\hline 22 & 1 & 0 \\
\hline 23 & 1 & 0 \\
\hline 24 & 6 & 0 \\
\hline 25 & 6 & 0 \\
\hline 26 & 6 & 0 \\
\hline 27 & 6 & 0 \\
\hline 28 & 6 & 0 \\
\hline 29 & 6 & 0 \\
\hline 30 & 1 & 0 \\
\hline 31 & 1 & 0 \\
\hline 32 & 8 & 0 \\
\hline 33 & 1 & 0 \\
\hline 34 & 8 & 0 \\
\hline 35 & 1 & 0 \\
\hline 36 & 6 & 0 \\
\hline 37 & 6 & 0 \\
\hline 38 & 6 & 0 \\
\hline 39 & 6 & 0 \\
\hline 40 & 6 & 0 \\
\hline 41 & 6 & 0 \\
\hline 42 & 6 & 0 \\
\hline 43 & 6 & 0 \\
\hline 44 & 1 & 0 \\
\hline 45 & 1 & 0 \\
\hline 46 & 1 & 0 \\
\hline 47 & 8 & 0 \\
\hline 48 & 8 & 0 \\
\hline 49 & 1 & 0 \\
\hline 50 & 1 & 0 \\
\hline 51 & 1 & 0 \\
\hline 52 & 8 & 0 \\
\hline 53 & 1 & 0 \\
\hline 54 & 6 & 0 \\
\hline 55 & 1 & 0 \\
\hline 56 & 1 & 0 \\
\hline 57 & 8 & 0 \\
\hline 58 & 1 & 0 \\
\hline 59 & 1 & 0 \\
\hline C-(S,R & piga & atec \\
\hline 1 & 6 & 0 \\
\hline 2 & 6 & 0 \\
\hline 3 & 6 & 0 \\
\hline 4 & 6 & 0 \\
\hline 5 & 6 & 0 \\
\hline 6 & 6 & 0 \\
\hline 7 & 1 & 0 \\
\hline 8 & 8 & 0 \\
\hline 9 & 1 & 0 \\
\hline 10 & 6 & 0 \\
\hline 11 & 1 & 0 \\
\hline 12 & 6 & 0 \\
\hline 13 & 6 & 0 \\
\hline 14 & 1 & 0 \\
\hline 15 & 1 & 0 \\
\hline 16 & 1 & 0 \\
\hline 17 & 8 & 0 \\
\hline 18 & 8 & 0 \\
\hline 19 & 6 & 0 \\
\hline 20 & 8 & 0 \\
\hline 21 & 8 & 0 \\
\hline 22 & 1 & 0 \\
\hline 23 & 1 & 0 \\
\hline
\end{tabular}




\begin{tabular}{|c|c|}
\hline 24 & 6 \\
\hline 25 & 6 \\
\hline 26 & 6 \\
\hline 27 & 6 \\
\hline 28 & 6 \\
\hline 29 & 6 \\
\hline 30 & 1 \\
\hline 31 & 1 \\
\hline 32 & 8 \\
\hline 33 & 1 \\
\hline 34 & 8 \\
\hline 35 & 1 \\
\hline 36 & 6 \\
\hline 37 & 6 \\
\hline 38 & 6 \\
\hline 39 & 6 \\
\hline 40 & 6 \\
\hline 41 & 6 \\
\hline 42 & 6 \\
\hline 43 & 6 \\
\hline 44 & 1 \\
\hline 45 & 1 \\
\hline 46 & 1 \\
\hline 47 & 8 \\
\hline 48 & 8 \\
\hline 49 & 1 \\
\hline 50 & 1 \\
\hline 51 & 1 \\
\hline 52 & 8 \\
\hline 53 & 1 \\
\hline 54 & 6 \\
\hline 55 & 1 \\
\hline 56 & 1 \\
\hline 57 & 8 \\
\hline 58 & 1 \\
\hline 59 & 8 \\
\hline 60 & 6 \\
\hline 61 & 6 \\
\hline 62 & 6 \\
\hline 63 & 6 \\
\hline 64 & 1 \\
\hline 65 & 6 \\
\hline 66 & 1 \\
\hline 67 & 6 \\
\hline 68 & 8 \\
\hline 69 & 1 \\
\hline 70 & 8 \\
\hline 71 & 1 \\
\hline 72 & 8 \\
\hline 73 & 1 \\
\hline 74 & 1 \\
\hline $5 \mathrm{OH}-($ & $-\mathrm{C}_{2}$ \\
\hline 1 & 6 \\
\hline 2 & 6 \\
\hline 3 & 6 \\
\hline 4 & 6 \\
\hline 5 & 6 \\
\hline 6 & 6 \\
\hline 7 & 1 \\
\hline 8 & 1 \\
\hline 9 & 1 \\
\hline 10 & 6 \\
\hline 11 & 1 \\
\hline 12 & 6 \\
\hline 13 & 6 \\
\hline 14 & 1 \\
\hline 15 & 1 \\
\hline 16 & 1 \\
\hline 17 & 8 \\
\hline 18 & 8 \\
\hline 19 & 1 \\
\hline 20 & 8 \\
\hline 21 & 8 \\
\hline 22 & 6 \\
\hline 23 & 1 \\
\hline 24 & 1 \\
\hline 25 & 1 \\
\hline 26 & 6 \\
\hline 27 & 6 \\
\hline 28 & 6 \\
\hline 29 & 6 \\
\hline 30 & 6 \\
\hline 31 & 6 \\
\hline 32 & 1 \\
\hline 33 & 1 \\
\hline 34 & 8 \\
\hline 35 & 1 \\
\hline 36 & 8 \\
\hline 37 & 1 \\
\hline 38 & 6 \\
\hline 39 & 6 \\
\hline 40 & 6 \\
\hline 41 & 6 \\
\hline 42 & 6 \\
\hline 43 & 6 \\
\hline 44 & 6 \\
\hline 45 & 6 \\
\hline
\end{tabular}




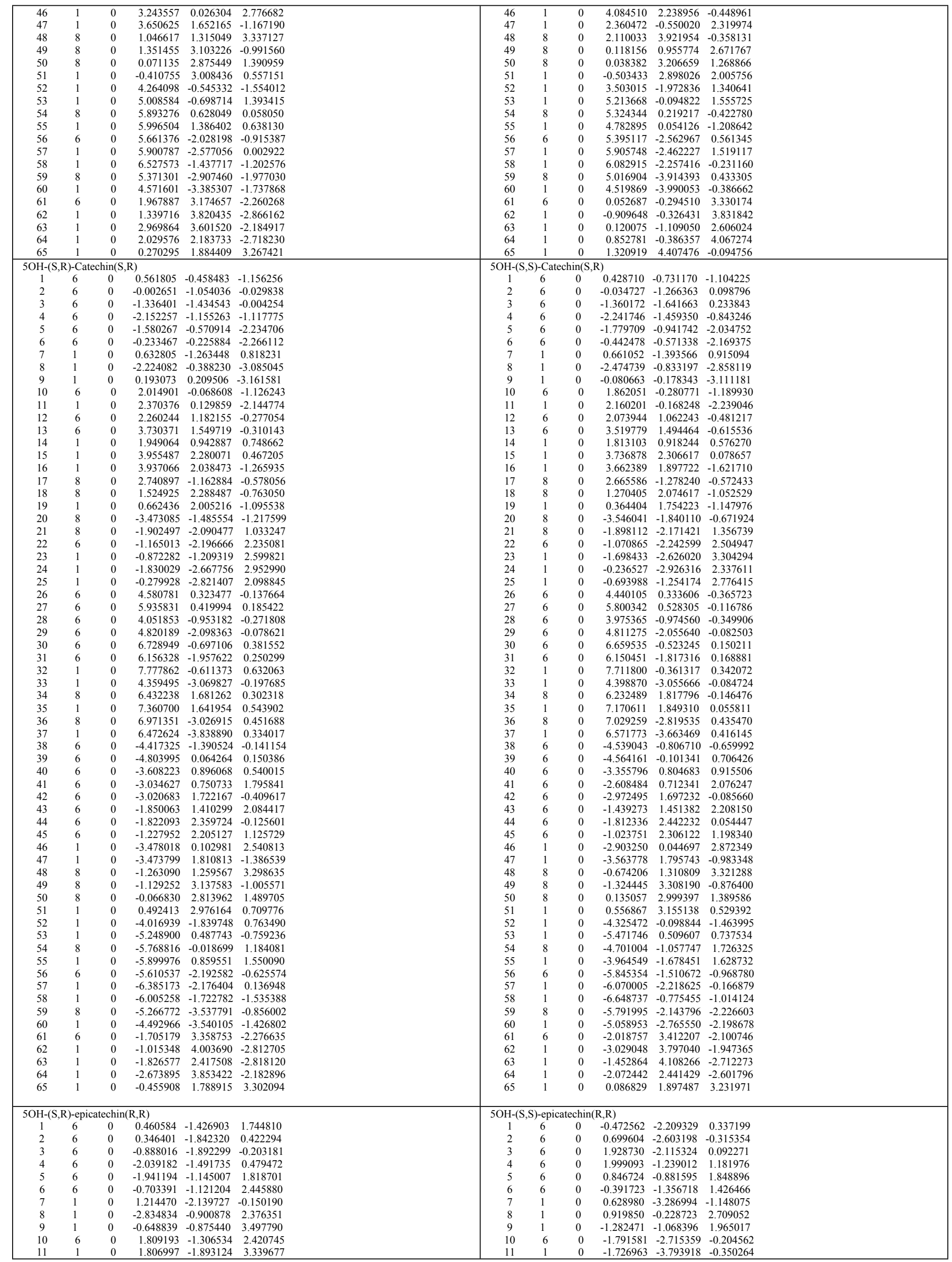




\begin{tabular}{|c|}
\hline 12 \\
\hline 13 \\
\hline 14 \\
\hline 15 \\
\hline 16 \\
\hline 17 \\
\hline 18 \\
\hline 19 \\
\hline 20 \\
\hline 21 \\
\hline 22 \\
\hline 23 \\
\hline 24 \\
\hline 25 \\
\hline 26 \\
\hline 27 \\
\hline 28 \\
\hline 29 \\
\hline 30 \\
\hline 31 \\
\hline 32 \\
\hline 33 \\
\hline 34 \\
\hline 35 \\
\hline 36 \\
\hline 37 \\
\hline 38 \\
\hline 39 \\
\hline 40 \\
\hline 41 \\
\hline 42 \\
\hline 43 \\
\hline 44 \\
\hline 45 \\
\hline 46 \\
\hline 47 \\
\hline 48 \\
\hline 49 \\
\hline 50 \\
\hline 51 \\
\hline 52 \\
\hline 53 \\
\hline 54 \\
\hline 55 \\
\hline 56 \\
\hline 57 \\
\hline 58 \\
\hline 59 \\
\hline 60 \\
\hline 61 \\
\hline 62 \\
\hline 63 \\
\hline 64 \\
\hline 65 \\
\hline \\
\hline \\
\hline $5 \mathrm{OH}-(\mathrm{S}$ \\
\hline 1 \\
\hline 2 \\
\hline 3 \\
\hline 4 \\
\hline 5 \\
\hline 6 \\
\hline 7 \\
\hline 8 \\
\hline 9 \\
\hline 10 \\
\hline 11 \\
\hline 12 \\
\hline 13 \\
\hline 14 \\
\hline 15 \\
\hline 16 \\
\hline 17 \\
\hline 18 \\
\hline 19 \\
\hline 20 \\
\hline 21 \\
\hline 22 \\
\hline 23 \\
\hline 24 \\
\hline 25 \\
\hline 26 \\
\hline 27 \\
\hline 28 \\
\hline 29 \\
\hline 30 \\
\hline 31 \\
\hline 32 \\
\hline 33 \\
\hline 34 \\
\hline 35 \\
\hline 36 \\
\hline 37 \\
\hline 38 \\
\hline 39 \\
\hline 40 \\
\hline 41 \\
\hline
\end{tabular}




\begin{tabular}{|c|}
\hline \\
\hline 42 \\
\hline 43 \\
\hline 44 \\
\hline 45 \\
\hline 46 \\
\hline 47 \\
\hline 48 \\
\hline 49 \\
\hline 50 \\
\hline 51 \\
\hline 52 \\
\hline 53 \\
\hline 54 \\
\hline 55 \\
\hline 56 \\
\hline 57 \\
\hline 58 \\
\hline 59 \\
\hline 60 \\
\hline 61 \\
\hline 62 \\
\hline 63 \\
\hline 64 \\
\hline 65 \\
\hline $5 \mathrm{OH}-\mathrm{C}$ \\
\hline 1 \\
\hline 2 \\
\hline 3 \\
\hline 4 \\
\hline 5 \\
\hline 6 \\
\hline 7 \\
\hline 8 \\
\hline 9 \\
\hline 10 \\
\hline 11 \\
\hline 12 \\
\hline 13 \\
\hline 14 \\
\hline 15 \\
\hline 16 \\
\hline 17 \\
\hline 18 \\
\hline 19 \\
\hline 20 \\
\hline 21 \\
\hline 22 \\
\hline 23 \\
\hline 24 \\
\hline 25 \\
\hline 26 \\
\hline 27 \\
\hline 28 \\
\hline 29 \\
\hline 30 \\
\hline 31 \\
\hline 32 \\
\hline 33 \\
\hline 34 \\
\hline 35 \\
\hline 36 \\
\hline 37 \\
\hline 38 \\
\hline 39 \\
\hline 40 \\
\hline 41 \\
\hline 42 \\
\hline 43 \\
\hline 44 \\
\hline 45 \\
\hline 46 \\
\hline 47 \\
\hline 48 \\
\hline 49 \\
\hline 50 \\
\hline 51 \\
\hline 52 \\
\hline 53 \\
\hline 54 \\
\hline 55 \\
\hline 56 \\
\hline 57 \\
\hline 58 \\
\hline 59 \\
\hline 60 \\
\hline 61 \\
\hline 62 \\
\hline 63 \\
\hline $5 \mathrm{OH}-($ \\
\hline 1 \\
\hline 2 \\
\hline 3 \\
\hline 4 \\
\hline 5 \\
\hline 6 \\
\hline 7 \\
\hline 8 \\
\hline
\end{tabular}




\begin{tabular}{|c|}
\hline 9 \\
\hline 10 \\
\hline 11 \\
\hline 12 \\
\hline 13 \\
\hline 14 \\
\hline 15 \\
\hline 16 \\
\hline 17 \\
\hline 18 \\
\hline 19 \\
\hline 20 \\
\hline 21 \\
\hline 22 \\
\hline 23 \\
\hline 24 \\
\hline 25 \\
\hline 26 \\
\hline 27 \\
\hline 28 \\
\hline 29 \\
\hline 30 \\
\hline 31 \\
\hline 32 \\
\hline 33 \\
\hline 34 \\
\hline 35 \\
\hline 36 \\
\hline 37 \\
\hline 38 \\
\hline 39 \\
\hline 40 \\
\hline 41 \\
\hline 42 \\
\hline 43 \\
\hline 44 \\
\hline 45 \\
\hline 46 \\
\hline 47 \\
\hline 48 \\
\hline 49 \\
\hline 50 \\
\hline 51 \\
\hline 52 \\
\hline 53 \\
\hline 54 \\
\hline 55 \\
\hline 56 \\
\hline 57 \\
\hline 58 \\
\hline 59 \\
\hline 60 \\
\hline 61 \\
\hline 62 \\
\hline 63 \\
\hline 64 \\
\hline 65 \\
\hline 66 \\
\hline 67 \\
\hline 68 \\
\hline 69 \\
\hline 70 \\
\hline 71 \\
\hline 72 \\
\hline 73 \\
\hline 74 \\
\hline 75 \\
\hline 76 \\
\hline 77 \\
\hline 78 \\
\hline G-(S,R) \\
\hline 1 \\
\hline 2 \\
\hline 3 \\
\hline 4 \\
\hline 5 \\
\hline 6 \\
\hline 7 \\
\hline 8 \\
\hline 9 \\
\hline 10 \\
\hline 11 \\
\hline 12 \\
\hline 13 \\
\hline 14 \\
\hline 15 \\
\hline 16 \\
\hline 17 \\
\hline 18 \\
\hline 19 \\
\hline 20 \\
\hline 21 \\
\hline 22 \\
\hline 23 \\
\hline 24 \\
\hline 25 \\
\hline 26 \\
\hline 27 \\
\hline
\end{tabular}




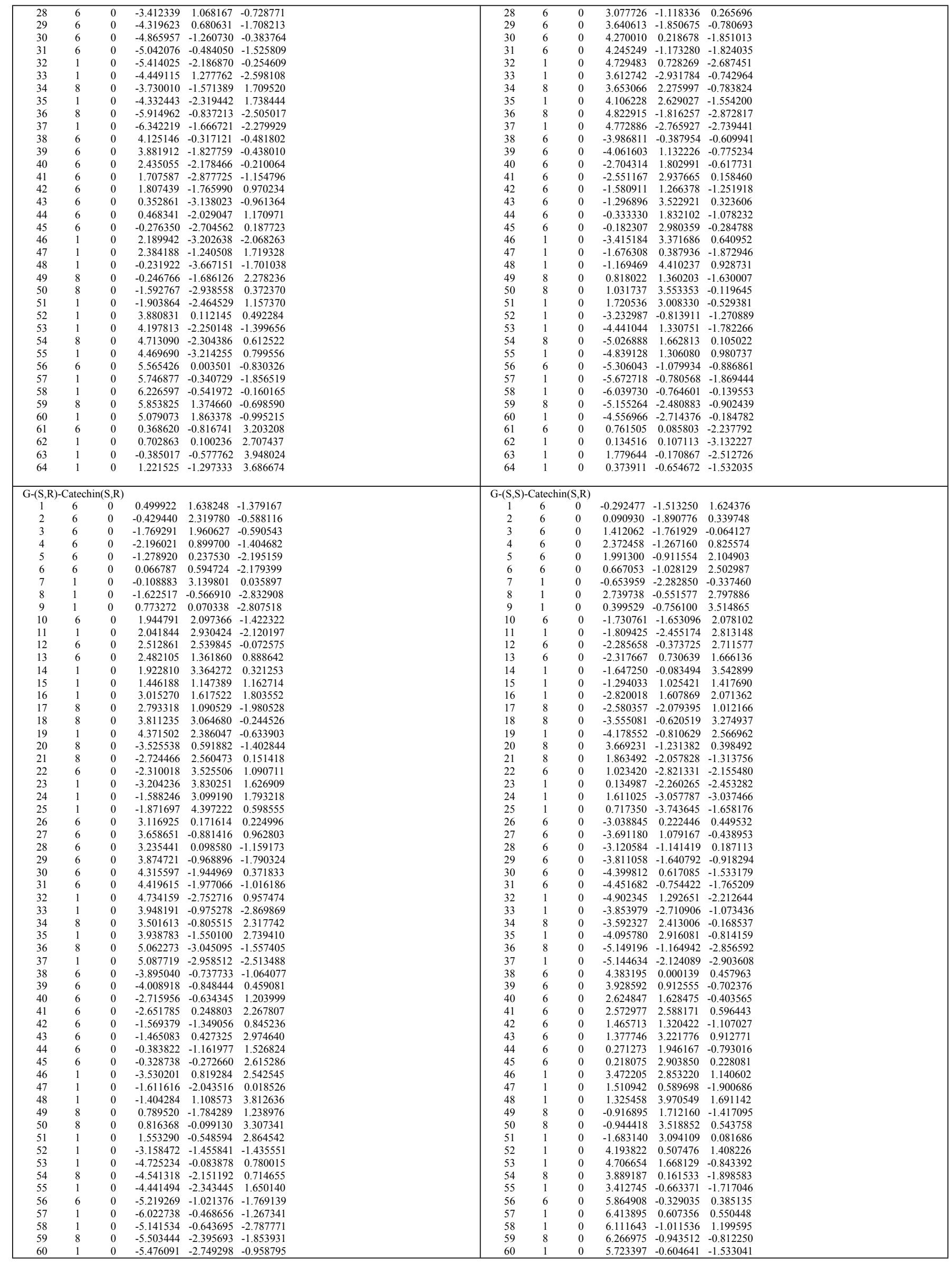




\begin{tabular}{|c|c|c|c|c|c|}
\hline $\begin{array}{l}61 \\
62 \\
63 \\
64\end{array}$ & $\begin{array}{l}6 \\
1 \\
1 \\
1\end{array}$ & $\begin{array}{l}0 \\
0 \\
0 \\
0\end{array}$ & $\begin{array}{l}0.856140 \\
1.886061 \\
0.197464 \\
0.582952\end{array}$ & $\begin{array}{l}-2.487601 \\
-2.810573 \\
-3.358671 \\
-1.829629\end{array}$ & $\begin{array}{r}0.012762 \\
-0.100931 \\
0.027282 \\
-0.816123\end{array}$ \\
\hline G-(S,F & pica & in $(R$ & & & \\
\hline 1 & 6 & 0 & 0.515400 & -0.847488 & 1.898763 \\
\hline 2 & 6 & 0 & 0.486961 & -1.427221 & 0.630679 \\
\hline 3 & 6 & 0 & -0.716587 & -1.755149 & 0.023839 \\
\hline 4 & 6 & 0 & -1.918990 & -1.519480 & 0.706298 \\
\hline 5 & 6 & 0 & -1.890861 & -0.984803 & 1.979452 \\
\hline 6 & 6 & 0 & -0.680208 & -0.637584 & 2.573352 \\
\hline 7 & 1 & 0 & 1.411644 & -1.625067 & 0.113760 \\
\hline 8 & 1 & 0 & -2.824009 & -0.851959 & 2.512487 \\
\hline 9 & 1 & 0 & -0.669549 & -0.227854 & 3.575577 \\
\hline 10 & 6 & 0 & 1.816252 & -0.391852 & 2.526387 \\
\hline 11 & 1 & 0 & 1.770139 & -0.569038 & 3.600616 \\
\hline 12 & 6 & 0 & 2.070872 & 1.108549 & 2.300447 \\
\hline 13 & 6 & 0 & 2.226761 & 1.371162 & 0.815524 \\
\hline 14 & 1 & 0 & 2.993799 & 1.359728 & 2.825317 \\
\hline 15 & 1 & 0 & 2.585195 & 2.392317 & 0.666396 \\
\hline 16 & 1 & 0 & 1.233133 & 1.297031 & 0.358934 \\
\hline 17 & 8 & 0 & 2.941365 & -1.132865 & 2.074253 \\
\hline 18 & 8 & 0 & 1.060272 & 1.903911 & 2.874170 \\
\hline 19 & 1 & 0 & 0.251854 & 1.751341 & 2.372423 \\
\hline 20 & 8 & 0 & -3.081899 & -1.888091 & 0.088892 \\
\hline 21 & 8 & 0 & -0.821529 & -2.294277 & -1.209311 \\
\hline 22 & 6 & 0 & 0.366488 & -2.418426 & -1.962788 \\
\hline 23 & 1 & 0 & 0.068357 & -2.794795 & -2.937084 \\
\hline 24 & 1 & 0 & 0.861300 & -1.449161 & -2.074874 \\
\hline 25 & 1 & 0 & 1.060235 & -3.122793 & -1.498613 \\
\hline 26 & 6 & 0 & 3.171609 & 0.376873 & 0.201553 \\
\hline 27 & 6 & 0 & 3.740839 & 0.578242 & -1.058224 \\
\hline 28 & 6 & 0 & 3.512615 & -0.792839 & 0.882126 \\
\hline 29 & 6 & 0 & 4.435585 & -1.697818 & 0.378148 \\
\hline 30 & 6 & 0 & 4.671043 & -0.308956 & -1.583405 \\
\hline 31 & 6 & 0 & 5.021312 & -1.434631 & -0.850119 \\
\hline 32 & 1 & 0 & 5.095346 & -0.110107 & -2.558673 \\
\hline 33 & 1 & 0 & 4.682743 & -2.591316 & 0.930911 \\
\hline 34 & 8 & 0 & 3.422990 & 1.651401 & -1.821484 \\
\hline 35 & 1 & 0 & 2.593844 & 2.065905 & -1.543866 \\
\hline 36 & 8 & 0 & 5.927135 & -2.337447 & -1.312607 \\
\hline 37 & 1 & 0 & 6.269791 & -2.042980 & -2.159694 \\
\hline 38 & 6 & 0 & -4.095538 & -0.900789 & -0.068175 \\
\hline 39 & 6 & 0 & -3.875864 & -0.124661 & -1.365775 \\
\hline 40 & 6 & 0 & -2.582122 & 0.655631 & -1.395552 \\
\hline 41 & 6 & 0 & -1.652488 & 0.465543 & -2.402330 \\
\hline 42 & 6 & 0 & -2.338065 & 1.616670 & -0.408487 \\
\hline 43 & 6 & 0 & -0.488603 & 1.232193 & -2.454759 \\
\hline 44 & 6 & 0 & -1.176719 & 2.359549 & -0.439612 \\
\hline 45 & 6 & 0 & -0.259755 & 2.185205 & -1.487199 \\
\hline 46 & 1 & 0 & -1.829262 & -0.290216 & -3.156381 \\
\hline 47 & 1 & 0 & -3.066957 & 1.771266 & 0.374798 \\
\hline 48 & 1 & 0 & 0.237592 & 1.108311 & -3.247034 \\
\hline 49 & 8 & 0 & -0.800674 & 3.293652 & 0.478849 \\
\hline 50 & 8 & 0 & 0.857682 & 2.966158 & -1.530472 \\
\hline 51 & 1 & 0 & 0.813075 & 3.588357 & -0.792624 \\
\hline 52 & 1 & 0 & -4.085389 & -0.209517 & 0.779005 \\
\hline 53 & 1 & 0 & -3.864874 & -0.849368 & -2.187947 \\
\hline 54 & 8 & 0 & -4.998696 & 0.741074 & -1.471307 \\
\hline 55 & 1 & 0 & -4.842716 & 1.357166 & -2.191291 \\
\hline 56 & 6 & 0 & -5.411498 & -1.655444 & -0.059619 \\
\hline 57 & 1 & 0 & -6.236666 & -0.953354 & -0.143255 \\
\hline 58 & 1 & 0 & -5.438483 & -2.331103 & -0.922668 \\
\hline 59 & 8 & 0 & -5.569719 & -2.360174 & 1.149203 \\
\hline 60 & 1 & 0 & -4.807989 & -2.940423 & 1.241006 \\
\hline 61 & 6 & 0 & -1.647703 & 3.492969 & 1.594016 \\
\hline 62 & 1 & 0 & -2.608077 & 3.907744 & 1.282501 \\
\hline 63 & 1 & 0 & -1.820209 & 2.550794 & 2.123176 \\
\hline 64 & 1 & 0 & -1.133696 & 4.189694 & 2.248371 \\
\hline G-(S,R & pica & in $(S$ & & & \\
\hline 1 & 6 & 0 & -0.123804 & -1.524846 & -0.659235 \\
\hline 2 & 6 & 0 & 0.564190 & $-0.642424-$ & -1.493944 \\
\hline 3 & 6 & 0 & 1.947276 & -0.582215 & -1.489688 \\
\hline 4 & 6 & 0 & 2.663612 & -1.387528 & -0.587026 \\
\hline 5 & 6 & 0 & 1.981078 & -2.293415 & 0.199526 \\
\hline 6 & 6 & 0 & 0.591525 & -2.368551 & 0.172461 \\
\hline 7 & 1 & 0 & -0.006089 & 0.008480 & -2.140753 \\
\hline 8 & 1 & 0 & 2.560753 & -2.914463 & 0.869640 \\
\hline 9 & 1 & 0 & 0.082778 & -3.057764 & 0.831225 \\
\hline 10 & 6 & 0 & -1.631094 & -1.506102 & -0.725130 \\
\hline 11 & 1 & 0 & -1.962650 & -1.811527 & -1.724925 \\
\hline 12 & 6 & 0 & -2.360554 & -2.361586 & 0.303991 \\
\hline 13 & 6 & 0 & -3.854940 & -2.310590 & 0.009213 \\
\hline 14 & 1 & 0 & -2.011274 & -3.393058 & 0.233562 \\
\hline 15 & 1 & 0 & -4.397496 & -2.700538 & 0.870230 \\
\hline 16 & 1 & 0 & -4.089429 & -2.963342 & -0.836046 \\
\hline 17 & 8 & 0 & -2.021304 & -0.137577 & -0.536122 \\
\hline 18 & 8 & 0 & -2.080789 & -1.904904 & 1.610863 \\
\hline 19 & 1 & 0 & -1.840447 & -0.969738 & 1.565550 \\
\hline 20 & 8 & 0 & 4.024233 & -1.338094 & -0.476174 \\
\hline 21 & 8 & 0 & 2.676995 & 0.221964 & -2.297771 \\
\hline 22 & 6 & 0 & 1.978587 & 1.083613 & -3.169573 \\
\hline 23 & 1 & 0 & 2.735234 & 1.648262 & -3.706402 \\
\hline 24 & 1 & 0 & 1.374786 & 0.516065 & -3.881349 \\
\hline 25 & 1 & 0 & 1.336350 & 1.766784 & -2.608540 \\
\hline 26 & 6 & 0 & -4.287357 & -0.899205 & -0.279241 \\
\hline
\end{tabular}




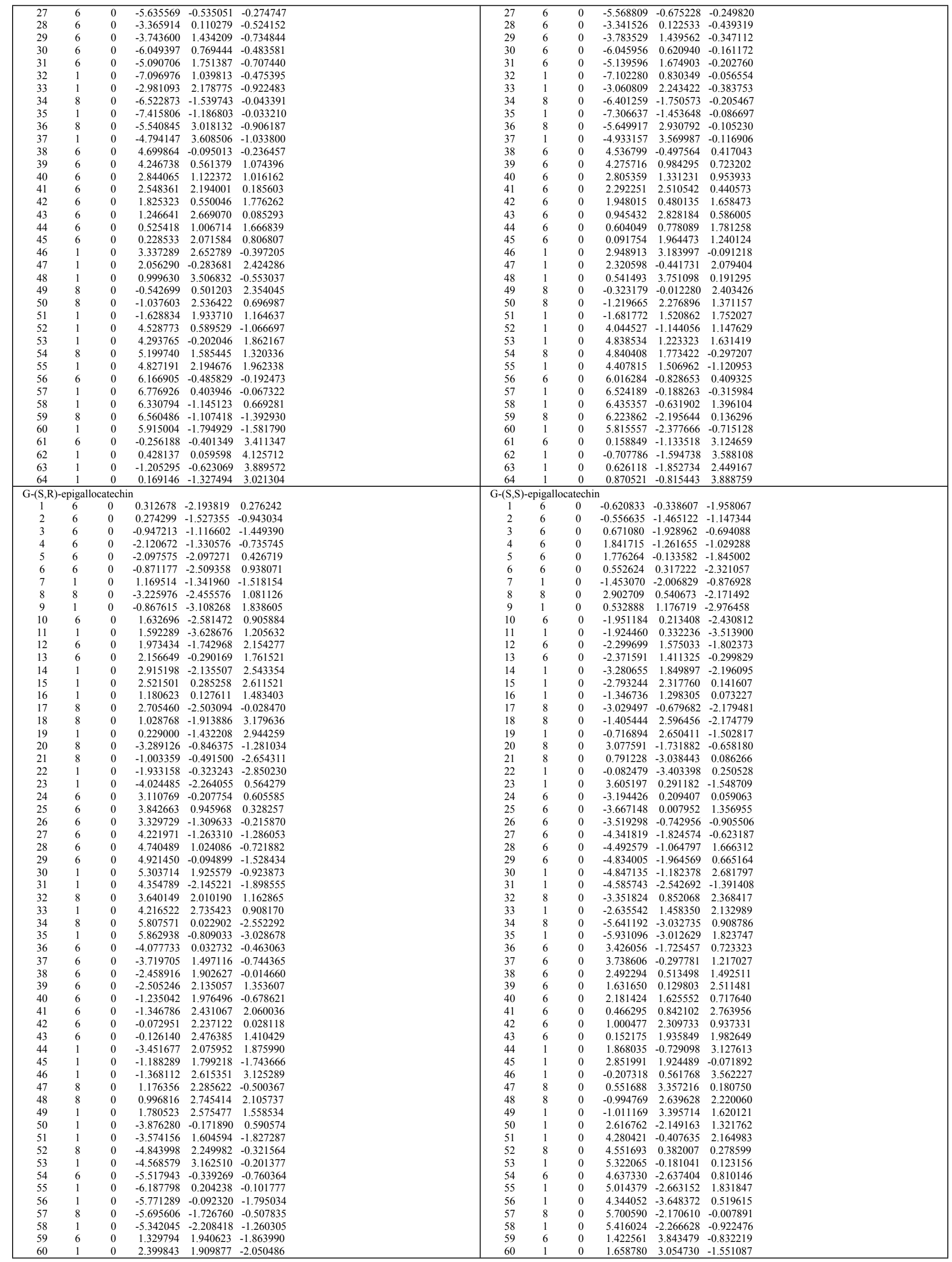




\begin{tabular}{|c|c|c|}
\hline 61 & 1 & 0 \\
\hline & & \\
\hline G-(S,R & piga & catecl \\
\hline 1 & 6 & 0 \\
\hline 2 & 6 & 0 \\
\hline 3 & 6 & 0 \\
\hline 4 & 6 & 0 \\
\hline 5 & 6 & 0 \\
\hline 6 & 6 & 0 \\
\hline 7 & 1 & 0 \\
\hline 8 & 8 & 0 \\
\hline 9 & 1 & 0 \\
\hline 10 & 6 & 0 \\
\hline 11 & 1 & 0 \\
\hline 12 & 6 & 0 \\
\hline 13 & 6 & 0 \\
\hline 14 & 1 & 0 \\
\hline 15 & 1 & 0 \\
\hline 16 & 1 & 0 \\
\hline 17 & 8 & 0 \\
\hline 18 & 8 & 0 \\
\hline 19 & 6 & 0 \\
\hline 20 & 8 & 0 \\
\hline 21 & 8 & 0 \\
\hline 22 & 1 & 0 \\
\hline 23 & 1 & 0 \\
\hline 24 & 6 & 0 \\
\hline 25 & 6 & 0 \\
\hline 26 & 6 & 0 \\
\hline 27 & 6 & 0 \\
\hline 28 & 6 & 0 \\
\hline 29 & 6 & 0 \\
\hline 30 & 1 & 0 \\
\hline 31 & 1 & 0 \\
\hline 32 & 8 & 0 \\
\hline 33 & 1 & 0 \\
\hline 34 & 8 & 0 \\
\hline 35 & 1 & 0 \\
\hline 36 & 6 & 0 \\
\hline 37 & 6 & 0 \\
\hline 38 & 6 & 0 \\
\hline 39 & 6 & 0 \\
\hline 40 & 6 & 0 \\
\hline 41 & 6 & 0 \\
\hline 42 & 6 & 0 \\
\hline 43 & 6 & 0 \\
\hline 44 & 1 & 0 \\
\hline 45 & 1 & 0 \\
\hline 46 & 1 & 0 \\
\hline 47 & 8 & 0 \\
\hline 48 & 8 & 0 \\
\hline 49 & 1 & 0 \\
\hline 50 & 1 & 0 \\
\hline 51 & 1 & 0 \\
\hline 52 & 8 & 0 \\
\hline 53 & 1 & 0 \\
\hline 54 & 6 & 0 \\
\hline 55 & 1 & 0 \\
\hline 56 & 1 & 0 \\
\hline 57 & 8 & 0 \\
\hline 58 & 1 & 0 \\
\hline 59 & 8 & 0 \\
\hline 60 & 6 & 0 \\
\hline 61 & 6 & 0 \\
\hline 62 & 6 & 0 \\
\hline 63 & 6 & 0 \\
\hline 64 & 1 & 0 \\
\hline 65 & 6 & 0 \\
\hline 66 & 1 & 0 \\
\hline 67 & 6 & 0 \\
\hline 68 & 8 & 0 \\
\hline 69 & 1 & 0 \\
\hline 70 & 8 & 0 \\
\hline 71 & 1 & 0 \\
\hline 72 & 8 & 0 \\
\hline 73 & 1 & 0 \\
\hline 74 & 6 & 0 \\
\hline 75 & 1 & 0 \\
\hline 76 & 1 & 0 \\
\hline 77 & 1 & 0 \\
\hline F(satu & d)-(? & $-\mathrm{Ca}$ \\
\hline 1 & 6 & 0 \\
\hline 2 & 6 & 0 \\
\hline 3 & 6 & 0 \\
\hline 4 & 6 & 0 \\
\hline 5 & 6 & 0 \\
\hline 6 & 6 & 0 \\
\hline 7 & 1 & 0 \\
\hline 8 & 1 & 0 \\
\hline 9 & 1 & 0 \\
\hline 10 & 6 & 0 \\
\hline 11 & 1 & 0 \\
\hline 12 & 6 & 0 \\
\hline 13 & 6 & 0 \\
\hline 14 & 1 & 0 \\
\hline 1 & 1 & 0 \\
\hline
\end{tabular}




\begin{tabular}{|c|}
\hline 16 \\
\hline 17 \\
\hline 18 \\
\hline 19 \\
\hline 20 \\
\hline 21 \\
\hline 22 \\
\hline 23 \\
\hline 24 \\
\hline 25 \\
\hline 26 \\
\hline 27 \\
\hline 28 \\
\hline 29 \\
\hline 30 \\
\hline 31 \\
\hline 32 \\
\hline 33 \\
\hline 34 \\
\hline 35 \\
\hline 36 \\
\hline 37 \\
\hline 38 \\
\hline 39 \\
\hline 40 \\
\hline 41 \\
\hline 42 \\
\hline 43 \\
\hline 44 \\
\hline 45 \\
\hline 46 \\
\hline 47 \\
\hline 48 \\
\hline 49 \\
\hline 50 \\
\hline 51 \\
\hline 52 \\
\hline 53 \\
\hline 54 \\
\hline 55 \\
\hline 56 \\
\hline 57 \\
\hline 58 \\
\hline 59 \\
\hline 60 \\
\hline 61 \\
\hline 62 \\
\hline 63 \\
\hline 64 \\
\hline 65 \\
\hline 66 \\
\hline 67 \\
\hline 68 \\
\hline 69 \\
\hline $\mathrm{F}$ (satur \\
\hline 1 \\
\hline 2 \\
\hline 3 \\
\hline 4 \\
\hline 5 \\
\hline 6 \\
\hline 7 \\
\hline 8 \\
\hline 9 \\
\hline 10 \\
\hline 11 \\
\hline 12 \\
\hline 13 \\
\hline 14 \\
\hline 15 \\
\hline 16 \\
\hline 17 \\
\hline 18 \\
\hline 19 \\
\hline 20 \\
\hline 21 \\
\hline 22 \\
\hline 23 \\
\hline 24 \\
\hline 25 \\
\hline 26 \\
\hline 27 \\
\hline 28 \\
\hline 29 \\
\hline 30 \\
\hline 31 \\
\hline 32 \\
\hline 33 \\
\hline 34 \\
\hline 35 \\
\hline 36 \\
\hline 37 \\
\hline 38 \\
\hline 39 \\
\hline 40 \\
\hline 41 \\
\hline 42 \\
\hline 43 \\
\hline
\end{tabular}




\begin{tabular}{|c|c|c|}
\hline 44 & 6 & 0 \\
\hline 45 & 6 & 0 \\
\hline 46 & 1 & 0 \\
\hline 47 & 1 & 0 \\
\hline 48 & 1 & 0 \\
\hline 49 & 8 & 0 \\
\hline 50 & 8 & 0 \\
\hline 51 & 1 & 0 \\
\hline 52 & 1 & 0 \\
\hline 53 & 1 & 0 \\
\hline 54 & 8 & 0 \\
\hline 55 & 1 & 0 \\
\hline 56 & 6 & 0 \\
\hline 57 & 8 & 0 \\
\hline 58 & 8 & 0 \\
\hline 59 & 6 & 0 \\
\hline 60 & 6 & 0 \\
\hline 61 & 6 & 0 \\
\hline 62 & 1 & 0 \\
\hline 63 & 1 & 0 \\
\hline 64 & 1 & 0 \\
\hline 65 & 1 & 0 \\
\hline 66 & 1 & 0 \\
\hline 67 & 1 & 0 \\
\hline 68 & 1 & 0 \\
\hline 69 & 1 & 0 \\
\hline $\mathrm{F}$ (satur & d)-( & -epi \\
\hline 1 & 6 & 0 \\
\hline 2 & 6 & 0 \\
\hline 3 & 6 & 0 \\
\hline 4 & 6 & 0 \\
\hline 5 & 6 & 0 \\
\hline 6 & 6 & 0 \\
\hline 7 & 1 & 0 \\
\hline 8 & 1 & 0 \\
\hline 9 & 1 & 0 \\
\hline 10 & 6 & 0 \\
\hline 11 & 1 & 0 \\
\hline 12 & 6 & 0 \\
\hline 13 & 6 & 0 \\
\hline 14 & 1 & 0 \\
\hline 15 & 1 & 0 \\
\hline 16 & 1 & 0 \\
\hline 17 & 8 & 0 \\
\hline 18 & 8 & 0 \\
\hline 19 & 1 & 0 \\
\hline 20 & 8 & 0 \\
\hline 21 & 8 & 0 \\
\hline 22 & 6 & 0 \\
\hline 23 & 1 & 0 \\
\hline 24 & 1 & 0 \\
\hline 25 & 1 & 0 \\
\hline 26 & 6 & 0 \\
\hline 27 & 6 & 0 \\
\hline 28 & 6 & 0 \\
\hline 29 & 6 & 0 \\
\hline 30 & 6 & 0 \\
\hline 31 & 6 & 0 \\
\hline 32 & 1 & 0 \\
\hline 33 & 1 & 0 \\
\hline 34 & 8 & 0 \\
\hline 35 & 1 & 0 \\
\hline 36 & 8 & 0 \\
\hline 37 & 1 & 0 \\
\hline 38 & 6 & 0 \\
\hline 39 & 6 & 0 \\
\hline 40 & 6 & 0 \\
\hline 41 & 6 & 0 \\
\hline 42 & 6 & 0 \\
\hline 43 & 6 & 0 \\
\hline 44 & 6 & 0 \\
\hline 45 & 6 & 0 \\
\hline 46 & 1 & 0 \\
\hline 47 & 1 & 0 \\
\hline 48 & 1 & 0 \\
\hline 49 & 8 & 0 \\
\hline 50 & 8 & 0 \\
\hline 51 & 1 & 0 \\
\hline 52 & 1 & 0 \\
\hline 53 & 1 & 0 \\
\hline 54 & 8 & 0 \\
\hline 55 & 1 & 0 \\
\hline 56 & 6 & 0 \\
\hline 57 & 8 & 0 \\
\hline 58 & 8 & 0 \\
\hline 59 & 6 & 0 \\
\hline 60 & 6 & 0 \\
\hline 61 & 6 & 0 \\
\hline 62 & 1 & 0 \\
\hline 63 & 1 & 0 \\
\hline 64 & 1 & 0 \\
\hline 65 & 1 & 0 \\
\hline 66 & 1 & 0 \\
\hline 67 & 1 & 0 \\
\hline 68 & 1 & 0 \\
\hline 69 & 1 & 0 \\
\hline
\end{tabular}




\begin{tabular}{|c|}
\hline F(satur \\
\hline 1 \\
\hline 2 \\
\hline 3 \\
\hline 4 \\
\hline 5 \\
\hline 6 \\
\hline 7 \\
\hline 8 \\
\hline 9 \\
\hline 10 \\
\hline 11 \\
\hline 12 \\
\hline 13 \\
\hline 14 \\
\hline 15 \\
\hline 16 \\
\hline 17 \\
\hline 18 \\
\hline 19 \\
\hline 20 \\
\hline 21 \\
\hline 22 \\
\hline 23 \\
\hline 24 \\
\hline 25 \\
\hline 26 \\
\hline 27 \\
\hline 28 \\
\hline 29 \\
\hline 30 \\
\hline 31 \\
\hline 32 \\
\hline 33 \\
\hline 34 \\
\hline 35 \\
\hline 36 \\
\hline 37 \\
\hline 38 \\
\hline 39 \\
\hline 40 \\
\hline 41 \\
\hline 42 \\
\hline 43 \\
\hline 44 \\
\hline 45 \\
\hline 46 \\
\hline 47 \\
\hline 48 \\
\hline 49 \\
\hline 50 \\
\hline 51 \\
\hline 52 \\
\hline 53 \\
\hline 54 \\
\hline 55 \\
\hline 56 \\
\hline 57 \\
\hline 58 \\
\hline 59 \\
\hline 60 \\
\hline 61 \\
\hline 62 \\
\hline 63 \\
\hline 64 \\
\hline 65 \\
\hline 66 \\
\hline 67 \\
\hline 68 \\
\hline 69 \\
\hline F(satur \\
\hline 1 \\
\hline 2 \\
\hline 3 \\
\hline 4 \\
\hline 5 \\
\hline 6 \\
\hline 7 \\
\hline 8 \\
\hline 9 \\
\hline 10 \\
\hline 11 \\
\hline 12 \\
\hline 13 \\
\hline 14 \\
\hline 15 \\
\hline 16 \\
\hline 17 \\
\hline 18 \\
\hline 19 \\
\hline 20 \\
\hline 21 \\
\hline 22 \\
\hline 23 \\
\hline 24 \\
\hline 25 \\
\hline 26 \\
\hline 27 \\
\hline
\end{tabular}




\begin{tabular}{|c|}
\hline 28 \\
\hline 29 \\
\hline 30 \\
\hline 31 \\
\hline 32 \\
\hline 33 \\
\hline 34 \\
\hline 35 \\
\hline 36 \\
\hline 37 \\
\hline 38 \\
\hline 39 \\
\hline 40 \\
\hline 41 \\
\hline 42 \\
\hline 43 \\
\hline 44 \\
\hline 45 \\
\hline 46 \\
\hline 47 \\
\hline 48 \\
\hline 49 \\
\hline 50 \\
\hline 51 \\
\hline 52 \\
\hline 53 \\
\hline 54 \\
\hline 55 \\
\hline 56 \\
\hline 57 \\
\hline 58 \\
\hline 59 \\
\hline 60 \\
\hline 61 \\
\hline 62 \\
\hline 63 \\
\hline 64 \\
\hline 65 \\
\hline 66 \\
\hline 67 \\
\hline $\mathrm{F}$ (satur \\
\hline 1 \\
\hline 2 \\
\hline 3 \\
\hline 4 \\
\hline 5 \\
\hline 6 \\
\hline 7 \\
\hline 8 \\
\hline 9 \\
\hline 10 \\
\hline 11 \\
\hline 12 \\
\hline 13 \\
\hline 14 \\
\hline 15 \\
\hline 16 \\
\hline 17 \\
\hline 18 \\
\hline 19 \\
\hline 20 \\
\hline 21 \\
\hline 22 \\
\hline 23 \\
\hline 24 \\
\hline 25 \\
\hline 26 \\
\hline 27 \\
\hline 28 \\
\hline 29 \\
\hline 30 \\
\hline 31 \\
\hline 32 \\
\hline 33 \\
\hline 34 \\
\hline 35 \\
\hline 36 \\
\hline 37 \\
\hline 38 \\
\hline 39 \\
\hline 40 \\
\hline 41 \\
\hline 42 \\
\hline 43 \\
\hline 44 \\
\hline 45 \\
\hline 46 \\
\hline 47 \\
\hline 48 \\
\hline 49 \\
\hline 50 \\
\hline 51 \\
\hline 52 \\
\hline 53 \\
\hline 54 \\
\hline 55 \\
\hline 56 \\
\hline 57 \\
\hline
\end{tabular}




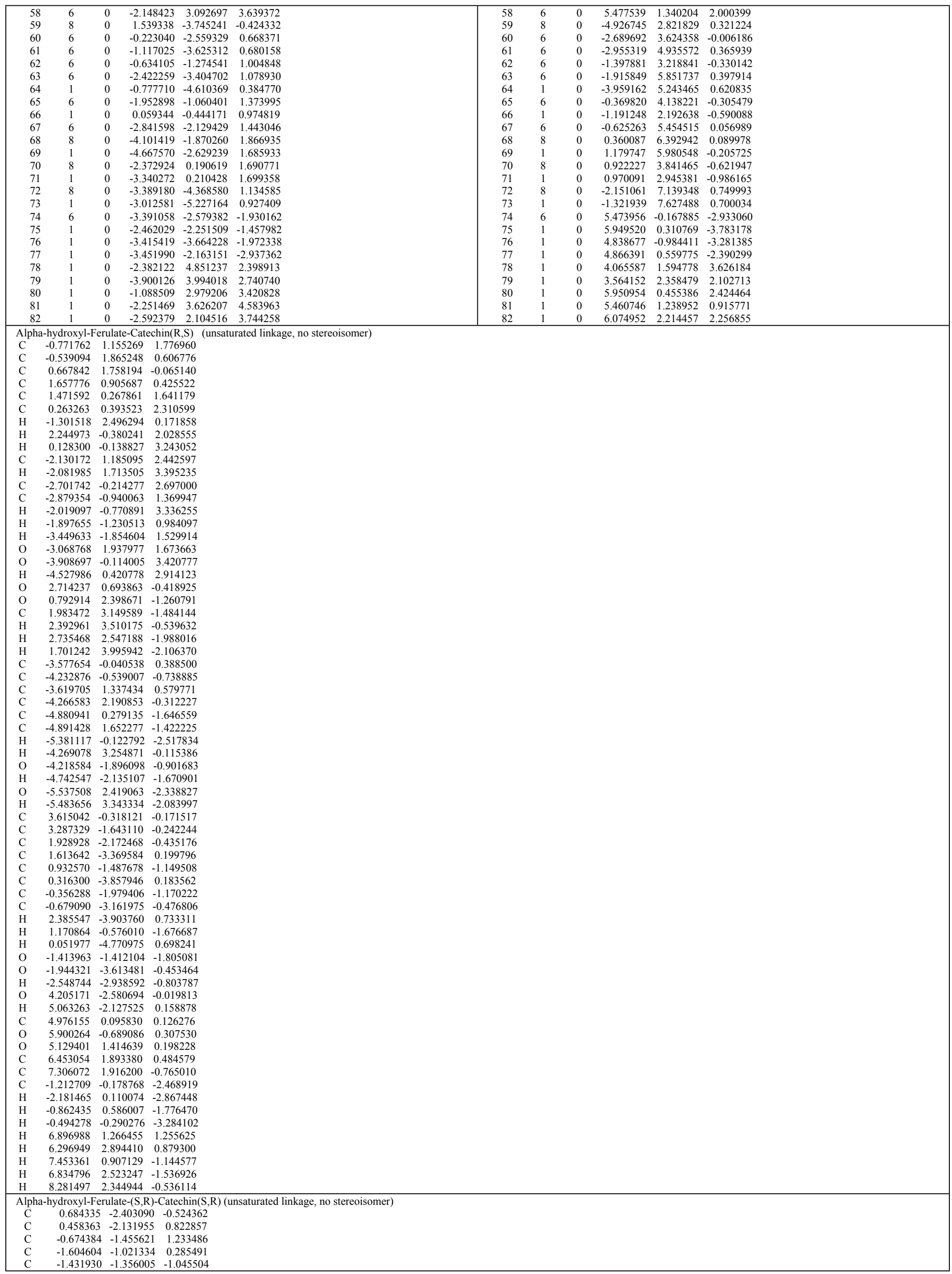




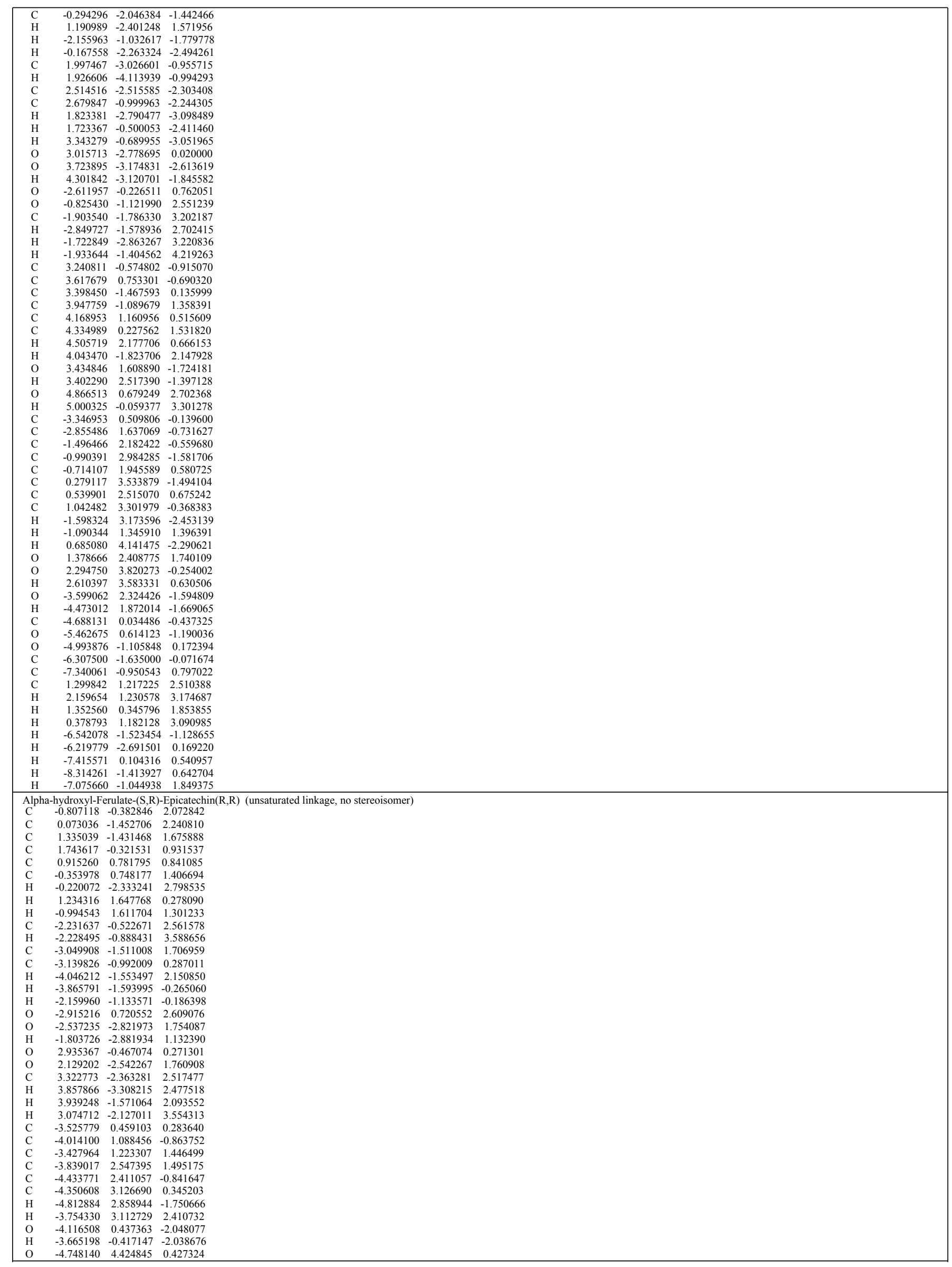




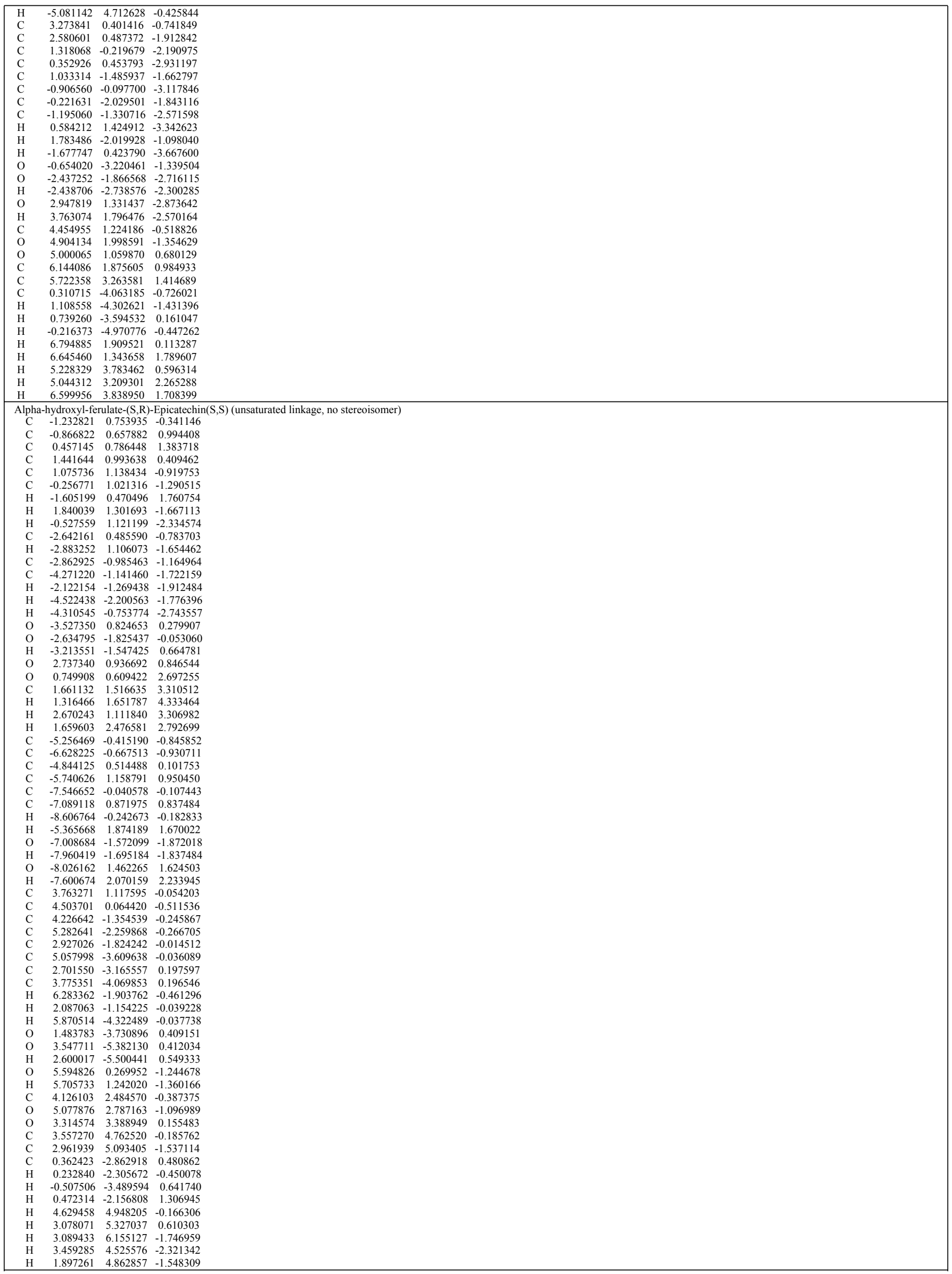




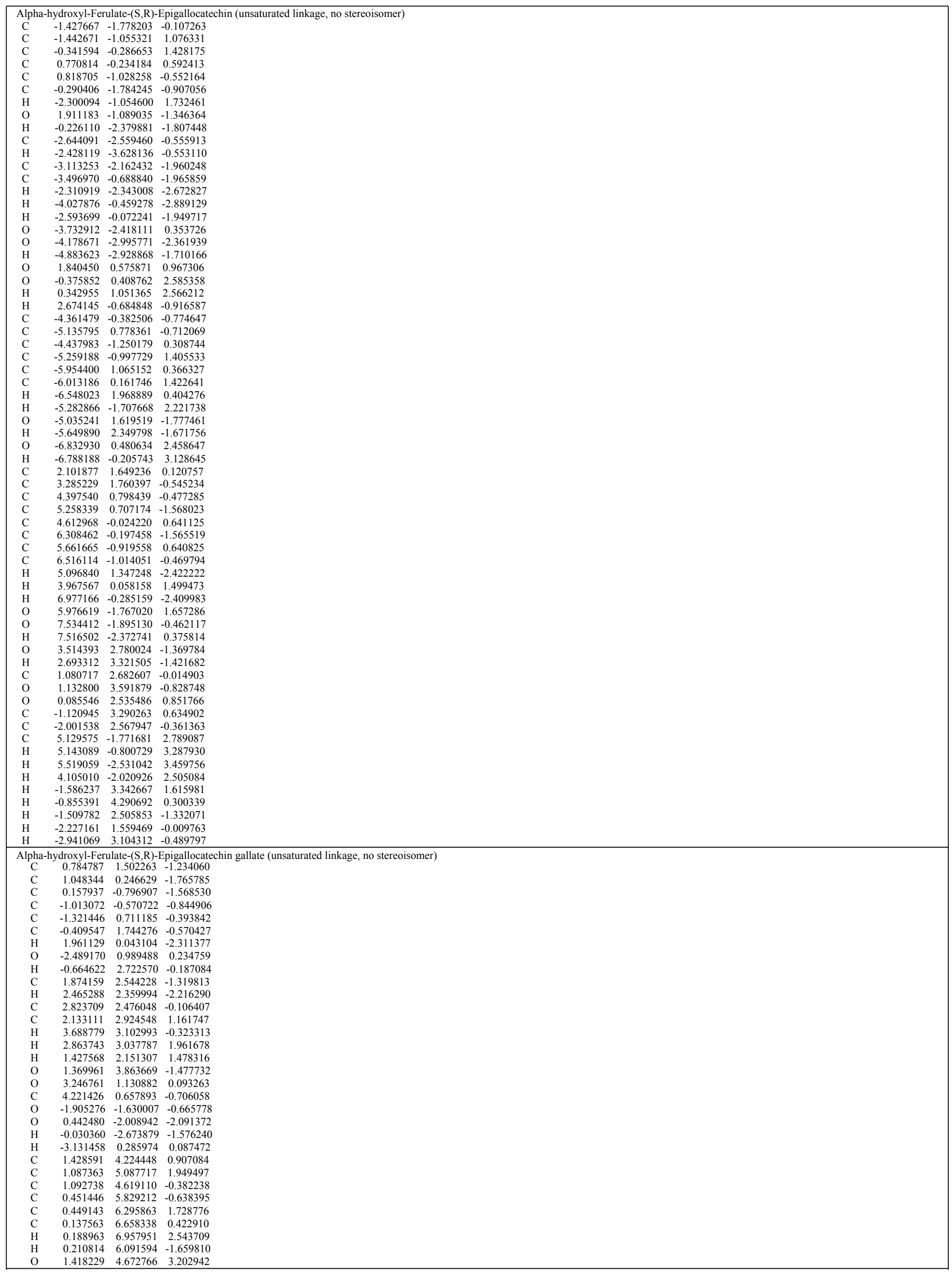




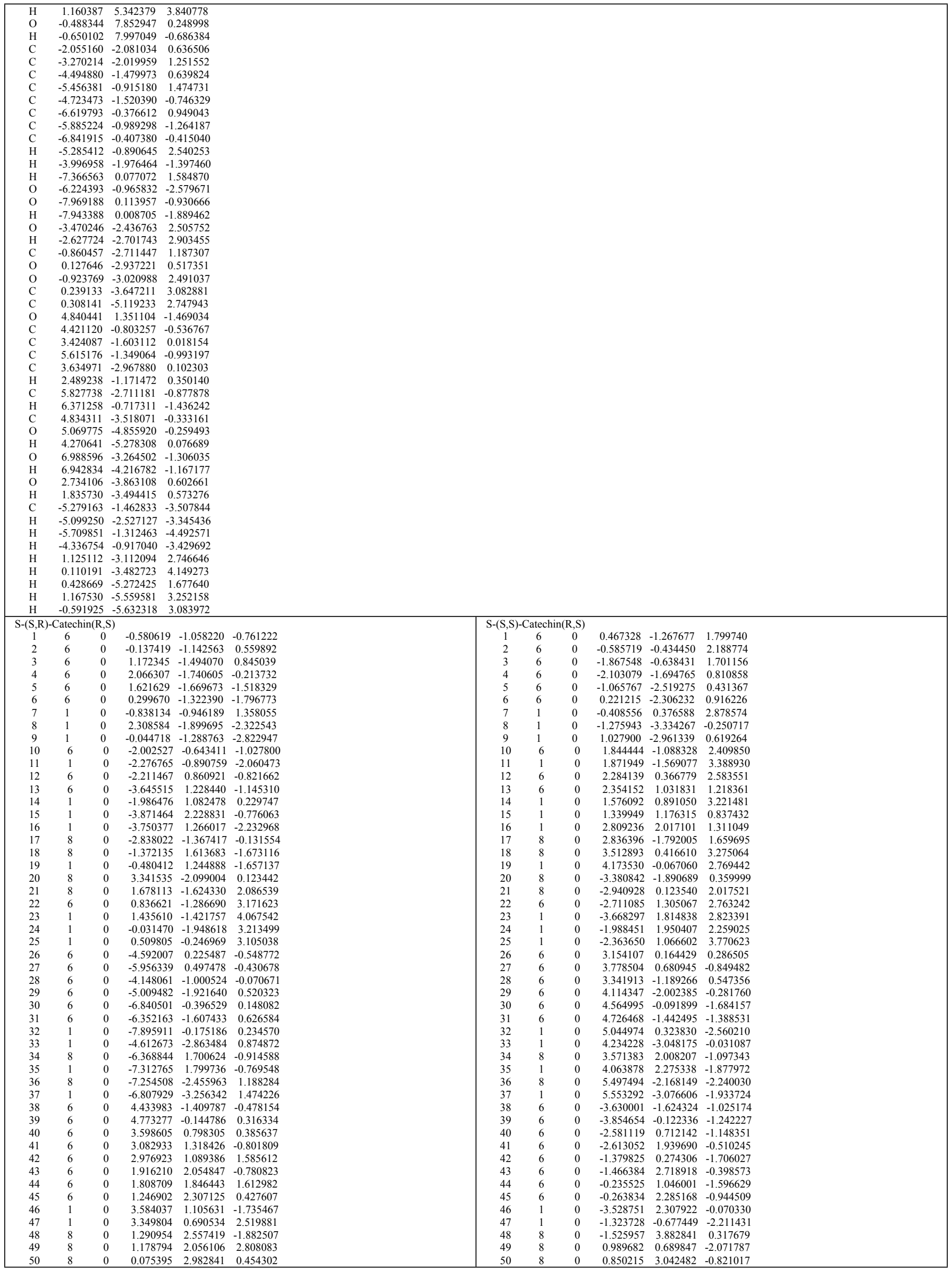




\begin{tabular}{|c|}
\hline 51 \\
\hline 52 \\
\hline 53 \\
\hline 54 \\
\hline 55 \\
\hline 56 \\
\hline 57 \\
\hline 58 \\
\hline 59 \\
\hline 60 \\
\hline 61 \\
\hline 62 \\
\hline 63 \\
\hline 64 \\
\hline 65 \\
\hline 66 \\
\hline 67 \\
\hline 68 \\
\hline S-(S,R \\
\hline 1 \\
\hline 2 \\
\hline 3 \\
\hline 4 \\
\hline 5 \\
\hline 6 \\
\hline 7 \\
\hline 8 \\
\hline 9 \\
\hline 10 \\
\hline 11 \\
\hline 12 \\
\hline 13 \\
\hline 14 \\
\hline 15 \\
\hline 16 \\
\hline 17 \\
\hline 18 \\
\hline 19 \\
\hline 20 \\
\hline 21 \\
\hline 22 \\
\hline 23 \\
\hline 24 \\
\hline 25 \\
\hline 26 \\
\hline 27 \\
\hline 28 \\
\hline 29 \\
\hline 30 \\
\hline 31 \\
\hline 32 \\
\hline 33 \\
\hline 34 \\
\hline 35 \\
\hline 36 \\
\hline 37 \\
\hline 38 \\
\hline 39 \\
\hline 40 \\
\hline 41 \\
\hline 42 \\
\hline 43 \\
\hline 44 \\
\hline 45 \\
\hline 46 \\
\hline 47 \\
\hline 48 \\
\hline 49 \\
\hline 50 \\
\hline 51 \\
\hline 52 \\
\hline 53 \\
\hline 54 \\
\hline 55 \\
\hline 56 \\
\hline 57 \\
\hline 58 \\
\hline 59 \\
\hline 60 \\
\hline 61 \\
\hline 62 \\
\hline 63 \\
\hline 64 \\
\hline 65 \\
\hline 66 \\
\hline 67 \\
\hline 68 \\
\hline S-(S,R) \\
\hline 1 \\
\hline 2 \\
\hline 3 \\
\hline 4 \\
\hline 5 \\
\hline 6 \\
\hline 7 \\
\hline 8 \\
\hline 9 \\
\hline 10 \\
\hline
\end{tabular}




\begin{tabular}{|c|}
\hline 11 \\
\hline 12 \\
\hline 13 \\
\hline 14 \\
\hline 15 \\
\hline 16 \\
\hline 17 \\
\hline 18 \\
\hline 19 \\
\hline 20 \\
\hline 21 \\
\hline 22 \\
\hline 23 \\
\hline 24 \\
\hline 25 \\
\hline 26 \\
\hline 27 \\
\hline 28 \\
\hline 29 \\
\hline 30 \\
\hline 31 \\
\hline 32 \\
\hline 33 \\
\hline 34 \\
\hline 35 \\
\hline 36 \\
\hline 37 \\
\hline 38 \\
\hline 39 \\
\hline 40 \\
\hline 41 \\
\hline 42 \\
\hline 43 \\
\hline 44 \\
\hline 45 \\
\hline 46 \\
\hline 47 \\
\hline 48 \\
\hline 49 \\
\hline 50 \\
\hline 51 \\
\hline 52 \\
\hline 53 \\
\hline 54 \\
\hline 55 \\
\hline 56 \\
\hline 57 \\
\hline 58 \\
\hline 59 \\
\hline 60 \\
\hline 61 \\
\hline 62 \\
\hline 63 \\
\hline 64 \\
\hline 65 \\
\hline 66 \\
\hline 67 \\
\hline 68 \\
\hline S-(S,R) \\
\hline 1 \\
\hline 2 \\
\hline 3 \\
\hline 4 \\
\hline 5 \\
\hline 6 \\
\hline 7 \\
\hline 8 \\
\hline 9 \\
\hline 10 \\
\hline 11 \\
\hline 12 \\
\hline 13 \\
\hline 14 \\
\hline 15 \\
\hline 16 \\
\hline 17 \\
\hline 18 \\
\hline 19 \\
\hline 20 \\
\hline 21 \\
\hline 22 \\
\hline 23 \\
\hline 24 \\
\hline 25 \\
\hline 26 \\
\hline 27 \\
\hline 28 \\
\hline 29 \\
\hline 30 \\
\hline 31 \\
\hline 32 \\
\hline 33 \\
\hline 34 \\
\hline 35 \\
\hline 36 \\
\hline 37 \\
\hline 38 \\
\hline 39 \\
\hline
\end{tabular}




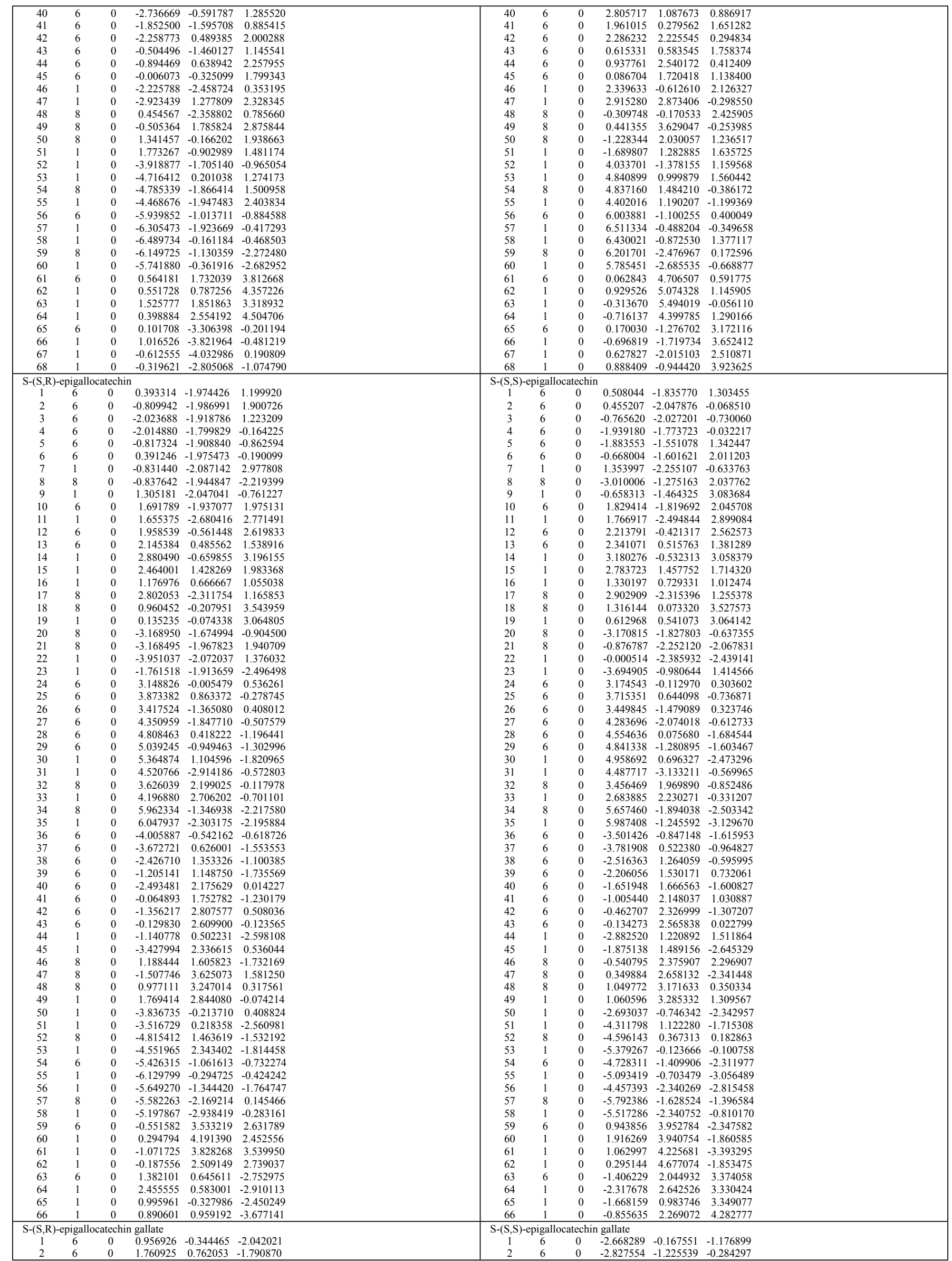




\begin{tabular}{|c|c|c|c|c|c|c|c|c|c|c|c|}
\hline 3 & 6 & 0 & 1.175830 & 2.015477 & -1.729068 & 3 & 6 & 0 & -1.794165 & -2.131621 & -0.116908 \\
\hline 4 & 6 & 0 & -0.198587 & 2.163697 & -1.873160 & 4 & 6 & 0 & -0.616884 & -2.024243 & -0.853595 \\
\hline 5 & 6 & 0 & -0.983651 & 1.075255 & -2.246549 & 5 & 6 & 0 & -0.486157 & -0.995209 & -1.785805 \\
\hline 6 & 6 & 0 & -0.396344 & -0.181779 & -2.312824 & 6 & 6 & 0 & -1.510507 & -0.064896 & -1.928260 \\
\hline 7 & 1 & 0 & 2.830017 & 0.675216 & -1.659869 & 7 & 1 & 0 & -3.734353 & -1.333256 & 0.298955 \\
\hline 8 & 8 & 0 & -2.298801 & 1.193382 & -2.537010 & 8 & 8 & 0 & 0.623186 & -0.842283 & -2.533891 \\
\hline 9 & 1 & 0 & -1.020315 & -1.017521 & -2.601520 & 9 & 1 & 0 & -1.367237 & 0.752003 & -2.623413 \\
\hline 10 & 6 & 0 & 1.510370 & -1.749169 & -1.994730 & 10 & 6 & 0 & -3.663228 & 0.965073 & -1.263696 \\
\hline 11 & 1 & 0 & 1.072385 & -2.334569 & -2.800871 & 11 & 1 & 0 & -3.365625 & 1.602881 & -2.092402 \\
\hline 12 & 6 & 0 & 1.200880 & -2.458923 & -0.658960 & 12 & 6 & 0 & -3.655549 & 1.830402 & 0.016669 \\
\hline 13 & 6 & 0 & 1.940808 & -1.796581 & 0.481679 & 13 & 6 & 0 & -4.423362 & 1.207422 & 1.169403 \\
\hline 14 & 1 & 0 & 1.474931 & -3.509558 & -0.770142 & 14 & 1 & 0 & -4.055545 & 2.811726 & -0.240123 \\
\hline 15 & 1 & 0 & 1.844198 & -2.396583 & 1.386507 & 15 & 1 & 0 & -4.671122 & 1.977241 & 1.899943 \\
\hline 16 & 1 & 0 & 1.449550 & -0.837277 & 0.674389 & 16 & 1 & 0 & -3.759145 & 0.507312 & 1.682938 \\
\hline 17 & 8 & 0 & 2.915698 & -1.792382 & -2.232035 & 17 & 8 & 0 & -4.976369 & 0.537284 & -1.605325 \\
\hline 18 & 8 & 0 & -0.168458 & -2.354347 & -0.300753 & 18 & 8 & 0 & -2.318977 & 1.965684 & 0.494904 \\
\hline 19 & 6 & 0 & -1.098363 & -3.031598 & -0.998639 & 19 & 6 & 0 & -1.426345 & 2.643248 & -0.247532 \\
\hline 20 & 8 & 0 & -0.721459 & 3.430312 & -1.722236 & 20 & 8 & 0 & 0.402687 & -2.922771 & -0.650262 \\
\hline 21 & 8 & 0 & 1.947992 & 3.114646 & -1.516784 & 21 & 8 & 0 & -1.840677 & -3.136428 & 0.813532 \\
\hline 22 & 1 & 0 & 1.368005 & 3.885045 & -1.546190 & 22 & 1 & 0 & -2.712598 & -3.153836 & 1.218188 \\
\hline 23 & 1 & 0 & -2.575803 & 2.119512 & -2.614069 & 23 & 1 & 0 & 1.243388 & -1.589026 & -2.438161 \\
\hline 24 & 6 & 0 & 3.376660 & -1.588178 & 0.124028 & 24 & 6 & 0 & -5.665079 & 0.512849 & 0.701043 \\
\hline 25 & 6 & 0 & 4.353318 & -1.384168 & 1.096233 & 25 & 6 & 0 & -6.663512 & 0.136057 & 1.602477 \\
\hline 26 & 6 & 0 & 3.782625 & -1.593244 & -1.205105 & 26 & 6 & 0 & -5.866961 & 0.205681 & -0.637435 \\
\hline 27 & 6 & 0 & 5.115552 & -1.401921 & -1.562832 & 27 & 6 & 0 & -7.021046 & -0.439175 & -1.078965 \\
\hline 28 & 6 & 0 & 5.684844 & -1.186968 & 0.775297 & 28 & 6 & 0 & -7.819970 & -0.505979 & 1.197243 \\
\hline 29 & 6 & 0 & 6.055012 & -1.201402 & -0.566596 & 29 & 6 & 0 & -7.989739 & -0.785810 & -0.155265 \\
\hline 30 & 1 & 0 & 6.439111 & -1.034037 & 1.535804 & 30 & 1 & 0 & -8.588415 & -0.791991 & 1.902826 \\
\hline 31 & 1 & 0 & 5.387566 & -1.412047 & -2.609819 & 31 & 1 & 0 & -7.133026 & -0.653764 & -2.133166 \\
\hline 32 & 8 & 0 & 3.926837 & -1.399075 & 2.396326 & 32 & 8 & 0 & -6.428903 & 0.436392 & 2.908937 \\
\hline 33 & 1 & 0 & 4.679368 & -1.294054 & 2.984175 & 33 & 1 & 0 & -7.185119 & 0.177060 & 3.440917 \\
\hline 34 & 8 & 0 & 7.371426 & -1.005680 & -0.839899 & 34 & 8 & 0 & -9.139697 & -1.416207 & -0.511301 \\
\hline 35 & 1 & 0 & 7.514356 & -1.046471 & -1.788666 & 35 & 1 & 0 & -9.154765 & -1.546696 & -1.462549 \\
\hline 36 & 6 & 0 & -1.756348 & 3.580067 & -0.738420 & 36 & 6 & 0 & 1.562910 & -2.370812 & -0.010213 \\
\hline 37 & 6 & 0 & -1.163625 & 4.001593 & 0.609062 & 37 & 6 & 0 & 2.824018 & -2.788072 & -0.789880 \\
\hline 38 & 6 & 0 & -0.517591 & 2.836485 & 1.326478 & 38 & 6 & 0 & 3.912494 & -1.777324 & -0.547953 \\
\hline 39 & 6 & 0 & 0.866252 & 2.703846 & 1.383680 & 39 & 6 & 0 & 3.786737 & -0.507981 & -1.117744 \\
\hline 40 & 6 & 0 & -1.321750 & 1.862923 & 1.895018 & 40 & 6 & 0 & 4.982638 & -2.063026 & 0.274625 \\
\hline 41 & 6 & 0 & 1.414143 & 1.590931 & 1.996212 & 41 & 6 & 0 & 4.736242 & 0.453331 & -0.841009 \\
\hline 42 & 6 & 0 & -0.773462 & 0.756467 & 2.541345 & 42 & 6 & 0 & 5.941307 & -1.089700 & 0.565040 \\
\hline 43 & 6 & 0 & 0.611178 & 0.620798 & 2.617022 & 43 & 6 & 0 & 5.812463 & 0.179149 & 0.017264 \\
\hline 44 & 1 & 0 & 1.506624 & 3.445477 & 0.930271 & 44 & 1 & 0 & 2.949998 & -0.281215 & -1.762453 \\
\hline 45 & 1 & 0 & -2.400815 & 1.946644 & 1.846370 & 45 & 1 & 0 & 5.111364 & -3.042617 & 0.716592 \\
\hline 46 & 8 & 0 & 2.739843 & 1.318129 & 2.080572 & 46 & 8 & 0 & 4.718355 & 1.728843 & -1.311309 \\
\hline 47 & 8 & 0 & -1.675806 & -0.117025 & 3.083668 & 47 & 8 & 0 & 6.941483 & -1.426757 & 1.422159 \\
\hline 48 & 8 & 0 & 1.202993 & -0.406162 & 3.272204 & 48 & 8 & 0 & 6.698322 & 1.160194 & 0.321539 \\
\hline 49 & 1 & 0 & 2.138577 & -0.448691 & 3.013748 & 49 & 1 & 0 & 6.417480 & 1.955597 & -0.146062 \\
\hline 50 & 1 & 0 & -2.272216 & 2.626870 & -0.602002 & 50 & 1 & 0 & 1.481893 & -1.280030 & -0.024969 \\
\hline 51 & 1 & 0 & -0.409962 & 4.776826 & 0.419251 & 51 & 1 & 0 & 3.157445 & -3.774050 & -0.450375 \\
\hline 52 & 8 & 0 & -2.250010 & 4.536870 & 1.344788 & 52 & 8 & 0 & 2.536911 & -2.844939 & -2.183369 \\
\hline 53 & 1 & 0 & -2.007665 & 4.561073 & 2.274102 & 53 & 1 & 0 & 1.910543 & -3.564360 & -2.317323 \\
\hline 54 & 6 & 0 & -2.731432 & 4.577283 & -1.335679 & 54 & 6 & 0 & 1.612783 & -2.851106 & 1.423719 \\
\hline 55 & 1 & 0 & -3.609430 & 4.665024 & -0.703652 & 55 & 1 & 0 & 1.542943 & -3.942614 & 1.438516 \\
\hline 56 & 1 & 0 & -2.264119 & 5.562499 & -1.421642 & 56 & 1 & 0 & 2.559001 & -2.552304 & 1.875876 \\
\hline 57 & 8 & 0 & -3.158436 & 4.101974 & -2.603987 & 57 & 8 & 0 & 0.579407 & -2.274059 & 2.201892 \\
\hline 58 & 1 & 0 & -2.457006 & 4.282675 & -3.235433 & 58 & 1 & 0 & -0.270757 & -2.617279 & 1.891861 \\
\hline 59 & 8 & 0 & -0.828177 & -3.798596 & -1.883903 & 59 & 8 & 0 & -1.734160 & 3.330704 & -1.185000 \\
\hline 60 & 6 & 0 & -2.459948 & -2.693884 & -0.518683 & 60 & 6 & 0 & -0.042214 & 2.423661 & 0.243331 \\
\hline 61 & 6 & 0 & -3.535132 & -3.458913 & -0.953427 & 61 & 6 & 0 & 0.932868 & 3.352621 & -0.095304 \\
\hline 62 & 6 & 0 & -2.640087 & -1.633212 & 0.366073 & 62 & 6 & 0 & 0.263717 & 1.300471 & 1.011527 \\
\hline 63 & 6 & 0 & -4.803993 & -3.172145 & -0.478610 & 63 & 6 & 0 & 2.226172 & 3.176216 & 0.369237 \\
\hline 64 & 1 & 0 & -3.385566 & -4.273962 & -1.646767 & 64 & 1 & 0 & 0.687081 & 4.209229 & -0.706456 \\
\hline 65 & 6 & 0 & -3.903316 & -1.374412 & 0.860837 & 65 & 6 & 0 & 1.561918 & 1.125523 & 1.461022 \\
\hline 66 & 1 & 0 & -1.806292 & -1.009898 & 0.655011 & 66 & 1 & 0 & -0.501892 & 0.574699 & 1.255413 \\
\hline 67 & 6 & 0 & -4.984564 & -2.137645 & 0.435927 & 67 & 6 & 0 & 2.538043 & 2.071184 & 1.154603 \\
\hline 68 & 8 & 0 & -6.241806 & -1.911040 & 0.899098 & 68 & 8 & 0 & 3.811085 & 1.958871 & 1.610511 \\
\hline 69 & 1 & 0 & -6.202075 & -1.186294 & 1.534385 & 69 & 1 & 0 & 3.914794 & 1.100875 & 2.041487 \\
\hline 70 & 8 & 0 & -4.184650 & -0.397622 & 1.769772 & 70 & 8 & 0 & 1.985607 & 0.083787 & 2.219787 \\
\hline 71 & 1 & 0 & -3.370081 & -0.147986 & 2.238353 & 71 & 1 & 0 & 1.284808 & -0.584145 & 2.332238 \\
\hline 72 & 8 & 0 & -5.867877 & -3.907885 & -0.881580 & 72 & 8 & 0 & 3.194150 & 4.072241 & 0.055632 \\
\hline 73 & 1 & 0 & -6.658500 & -3.567233 & -0.448815 & 73 & 1 & 0 & 4.032887 & 3.733792 & 0.391149 \\
\hline 74 & 6 & 0 & -1.306371 & -1.478360 & 3.325503 & 74 & 6 & 0 & 8.270007 & -1.073232 & 1.054371 \\
\hline 75 & 1 & 0 & -0.735337 & -1.561636 & 4.245650 & 75 & 1 & 0 & 8.498124 & -0.047245 & 1.332401 \\
\hline 76 & 1 & 0 & -2.245584 & -2.021013 & 3.409742 & 76 & 1 & 0 & 8.923099 & -1.758597 & 1.588827 \\
\hline 77 & 1 & 0 & -0.727893 & -1.877448 & 2.493350 & 77 & 1 & 0 & 8.415032 & -1.196398 & -0.020495 \\
\hline 78 & 6 & 0 & 3.630000 & 2.104157 & 1.309373 & 78 & 6 & 0 & 3.747391 & 2.045495 & -2.297890 \\
\hline 79 & 1 & 0 & 4.599451 & 1.618111 & 1.378731 & 79 & 1 & 0 & 3.897501 & 3.090963 & -2.546136 \\
\hline 80 & 1 & 0 & 3.309364 & 2.144642 & 0.268158 & 80 & 1 & 0 & 3.888922 & 1.419870 & -3.180867 \\
\hline 81 & 1 & 0 & 3.696120 & 3.118287 & 1.709990 & 81 & 1 & 0 & 2.737146 & 1.909080 & -1.908222 \\
\hline
\end{tabular}

